\title{
Feasibility of Providing Ubiquitous High Data Rate Coverage in Cellular Fixed Relay Networks
}

\author{
By \\ Ahmet Hakan Bolukbasi, B.S.
}

A thesis submitted to

The Faculty of Graduate Studies and Research

In partial fulfillment of

The requirements of the degree of

Master of Applied Science

Ottawa- Carleton Institute for Electrical and Computer Engineering

Department of Systems and Computer Engineering

Carleton University

Ottawa, Ontario

(C) Copyright 2005, Ahmet Hakan Bolukbasi 


$\begin{array}{ll}\begin{array}{l}\text { Library and } \\ \text { Archives Canada }\end{array} & \begin{array}{l}\text { Bibliothèque et } \\ \text { Archives Canada }\end{array} \\ \begin{array}{l}\text { Published Heritage } \\ \text { Branch }\end{array} & \begin{array}{l}\text { Direction du } \\ \text { Patrimoine de l'édition }\end{array} \\ \begin{array}{l}\text { 395 Wellington Street } \\ \text { Ottawa ON K1A ON4 }\end{array} & \begin{array}{l}\text { 395, rue Wellington } \\ \text { Ottawa ON K1A ON4 } \\ \text { Canada }\end{array}\end{array}$

Your file Votre référence

ISBN: 0-494-00735-4

Our file Notre référence

ISBN: 0-494-00735-4

NOTICE:

The author has granted a nonexclusive license allowing Library and Archives Canada to reproduce, publish, archive, preserve, conserve, communicate to the public by telecommunication or on the Internet, loan, distribute and sell theses worldwide, for commercial or noncommercial purposes, in microform, paper, electronic and/or any other formats.

The author retains copyright ownership and moral rights in this thesis. Neither the thesis nor substantial extracts from it may be printed or otherwise reproduced without the author's permission.
AVIS:

L'auteur a accordé une licence non exclusive permettant à la Bibliothèque et Archives Canada de reproduire, publier, archiver, sauvegarder, conserver, transmettre au public par télécommunication ou par l'Internet, prêter, distribuer et vendre des thèses partout dans le monde, à des fins commerciales ou autres, sur support microforme, papier, électronique et/ou autres formats.

L'auteur conserve la propriété du droit d'auteur et des droits moraux qui protège cette thèse. $\mathrm{Ni}$ la thèse ni des extraits substantiels de celle-ci ne doivent être imprimés ou autrement reproduits sans son autorisation.
In compliance with the Canadian

Privacy Act some supporting forms may have been removed from this thesis.

While these forms may be included in the document page count, their removal does not represent any loss of content from the thesis.
Conformément à la loi canadienne sur la protection de la vie privée, quelques formulaires secondaires ont été enlevés de cette thèse.

Bien que ces formulaires aient inclus dans la pagination, il n'y aura aucun contenu manquant.

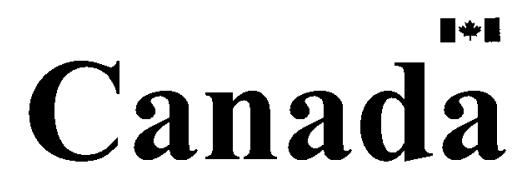




\begin{abstract}
Future wireless systems are intended to provide high data rates in order to support multimedia applications. This goal may require some fundamental changes in the existing network structure. Towards that end, due to its cost effective nature, multi-hop relaying is considered as one of the promising solutions for the requirements of the future networks.
\end{abstract}

This thesis studies the feasibility of ubiquitous high data rate coverage in Cellular Fixed Relay Networks (CFRN). Firstly, the capacity of CFRN is found through analytical derivations and compared to that of Pico-Cellular Networks (PCN), which is another candidate for future wireless networks, and Conventional Cellular Networks (CCN). Then, a composite multi-hop link is presented, where all the links in the same composite multi-hop link use orthogonal channels in order to prevent excessive in-cell interference. With the help of analytical derivations, the closest distance from the Central Node (CN) where $n$-hop communication can be utilized is found. Furthermore, the optimum number and position of the first tier Relay Stations (RSs) are found for ubiquitous high data rate coverage. Finally, a series of simulations are performed and performance of CFRN is compared to that of $\mathrm{CCN}$, where the performance criteria are: spectral efficiency distribution/coverage, outage, and average spectral efficiency. It is shown that, with 2hop relaying, $70 \%$ of the users can be provided with high data rate and the outage can be reduced to $0.25 \%$. In summary, CFRN can be cost effective solution to the high data rate coverage problem of $4 \mathrm{G}$ networks. If the objective is to provide close to $100 \%$ high data rate coverage, then, based on the cell size, propagation conditions, and the transmit powers of the CN and the RSs, more than one tier (too many) RSs may be necessary. 
However, if the $100 \%$ high data rate coverage requirement is relaxed, with sufficiently high RS transmit power, one tier with not too many RSs may be sufficient. In such a case, the outage reduction is still prominent. 


\section{Acknowledgments}

First and foremost, I would like to express my sincerest appreciation to my thesis cosupervisors Prof. Halim Yanikomeroglu and Prof. Falconer for their instruction, guidance and experience. Their profound knowledge and passion for research have greatly enhanced my enjoyment of this research.

In addition, I am grateful to Dr. Shalini Periyalwar for taking time to support me with her insightful knowledge ever since the beginning of this research. I also thankfully acknowledge the financial support provided by the Nortel Networks and Carleton University. As well, I would like to express my gratitude to the wonderful ladies from the departmental office who reminded me all the deadlines and solved all my registration problems immediately.

I also would like to recognize my parents for their continual encouragement and support.

I would like to thank to Mr. Luciano Pavarotti, Andrea Bocelli, Ludwig Van Beethoven, Wolfgang Amadeus Mozart, Antonio Vivaldi, Tom Waits, 3 Tenors, Bob Marley, and Mrs. Sarah Brightman for helping me isolating my mind from this world whenever necessary.

Last but not the least, I would like to thank my favorite soccer team Besiktas JK for being the Turkish Premier League Champions in $2003,100^{\text {th }}$ year anniversary of the foundation of the club. 


\section{Table of Contents}

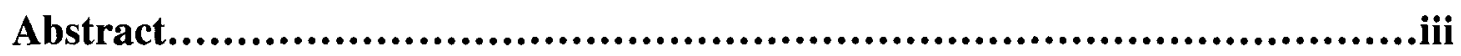

Acknowledgements....................................................................v

Table of Contents.......................................................................vi

List of Figures and Tables...........................................................

List of Acronyms.......................................................................

List of Symbols........................................................................

CHAPTER 1: INTRODUCTION …………………….................................. 1

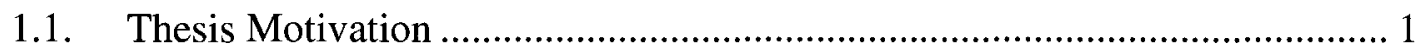

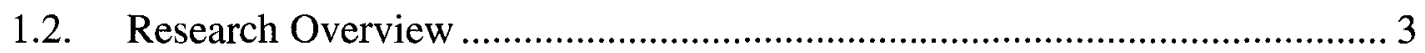

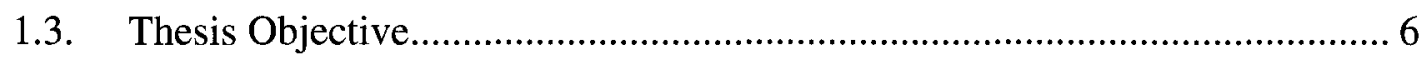

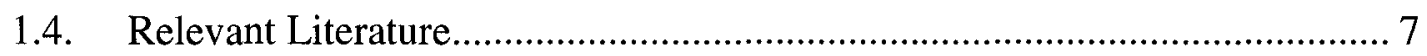

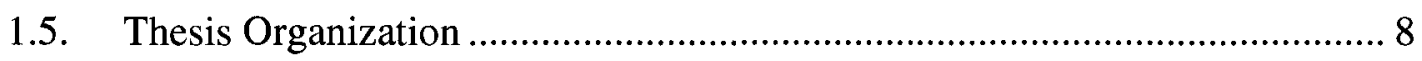

CHAPTER 2: Capacity of cellular fixed relay networks .......................................... 10

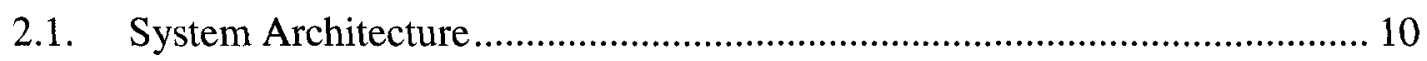

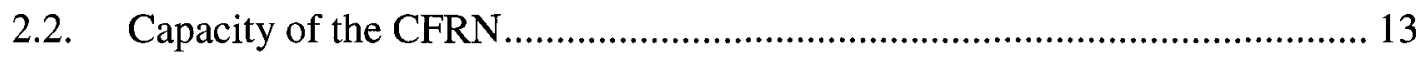

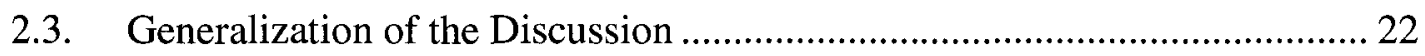

2.4. Capacity Comparison of CFRN and Pico-Cellular Networks (PCN) ........... 25

2.5. Generalization of (2.17) for Orthogonal Channels ..................... 27 


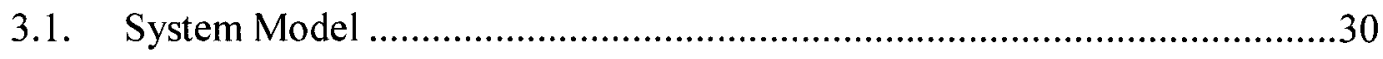

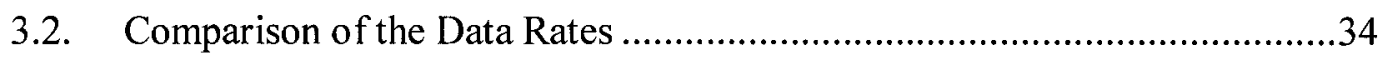

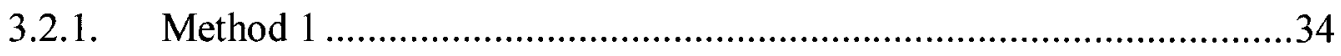

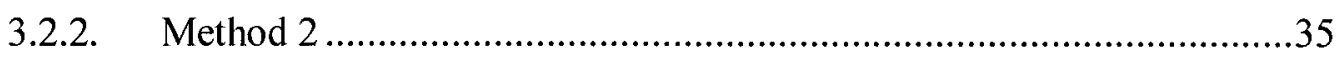

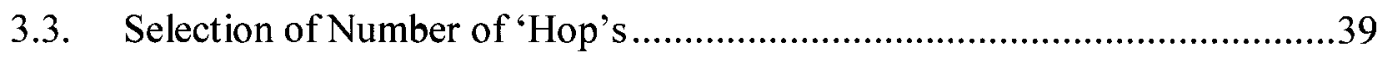

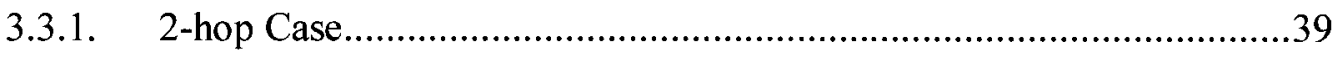

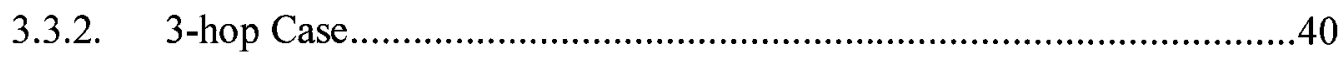

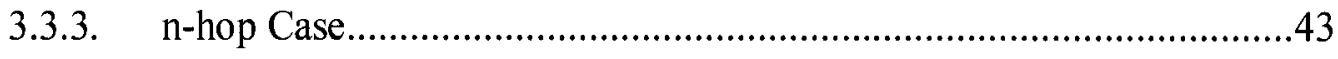

3.4. Finding the Radii of Spectral Efficiency Circles .................................44

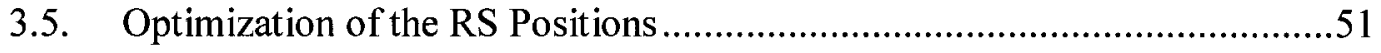

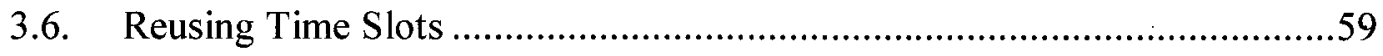

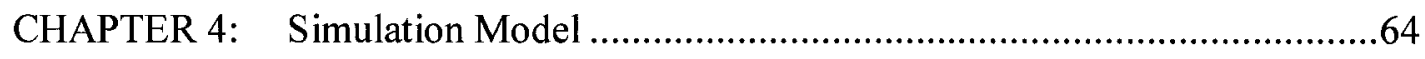

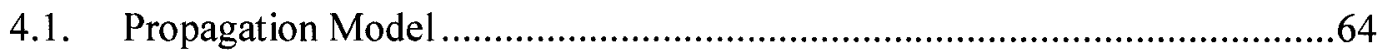

4.2. Environment and Parameters Assumptions .....................................65

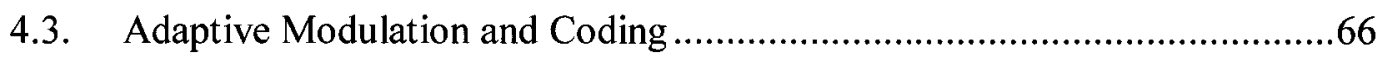

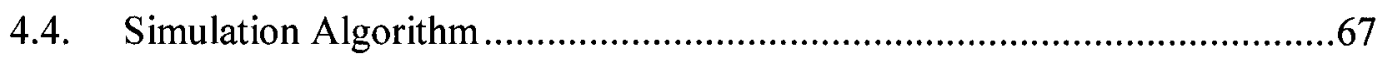

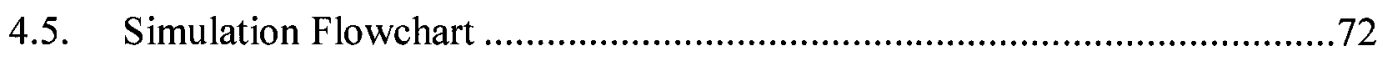

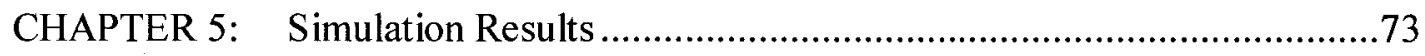




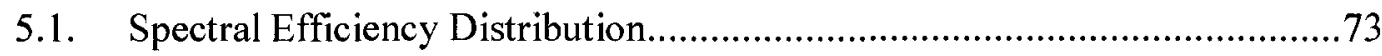

5.2. Coverage at Various SE Levels with respect to Number of RSs ............80

5.3. Average Spectral Efficiency for Non-relaying and Relaying Cases .............83

5.4. Outage Comparison for Non-relaying and Relaying Cases........................85

5.5. Coverage at Various SE Levels wrt RS Positions for 30 RSs .....................86

5.6. Coverage at Various SE Levels wrt ISF for 30 RSs ...............................89

5.7. Coverage at Various SE Levels wrt RS Transmit Power ...........................91

5.8. Adaptive Modulation and Coding Histogram........................................93

CHAPTER 6: Conclusions and Discussions .....................................................95

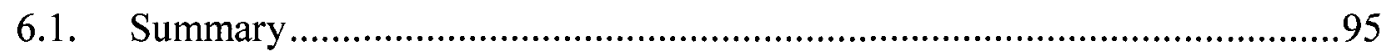

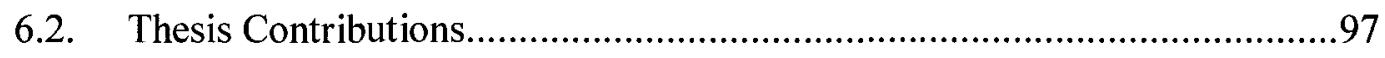

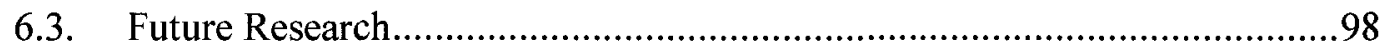

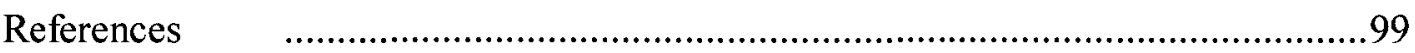

Appendix A - Definitions of Some Terms in Tree Structure ................................101

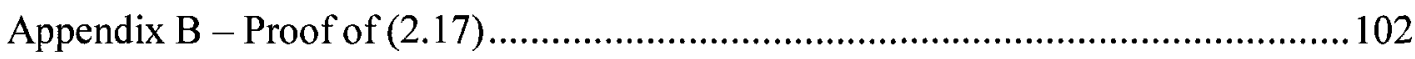

Appendix C - All Orthogonal Channel Groups for All the Links in a Tree..............103

Appendix D - Details of Ideal 2-hop Case ..................................................... 104 


\section{List of Figures and Tables}

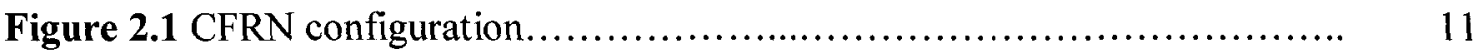

Figure 2.2 Central node and a tree in a binary tree structure $\ldots \ldots \ldots \ldots \ldots \ldots \ldots \ldots \ldots \ldots \ldots$

Figure 2.3 Available frequency spectrum................................ 15

Figure 2.4 Assigned channel groups for a tree of 7 nodes...................... 20

Figure 2.5 General structure of the CFRN $\ldots \ldots \ldots \ldots \ldots \ldots \ldots \ldots \ldots \ldots \ldots \ldots \ldots \ldots \ldots, 22$

Figure 2.6 Assigned channel group groups for a tree......................... 23

Figure $3.1 n$-hop communication system architecture........................ 29

Figure 3.2 Nodes and connections in the $n$-hop communication................ 31

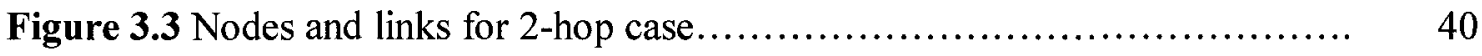

Figure 3.4 Nodes and links for 3 -hop Case.............................. $\quad 42$

Table 3.1 Possible links for CN-MS communication........................... 42

Figure 3.5 Spectral efficiency circles for $a_{\max }=6 \mathrm{bits} / \mathrm{sec} / \mathrm{Hz} \ldots \ldots \ldots \ldots \ldots \ldots \ldots \ldots . \quad 44$

Table 3.2 Relation of all combinations, required $S I N R$ and spectral efficiency that

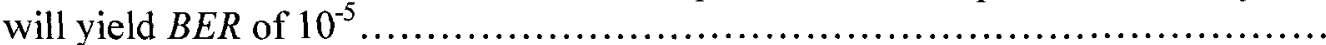

46

Figure 3.6 Spectral efficiency vs. SINR graph ............................ 47

Table 3.3 Spectral efficiency Circles radii ratios ........................... 50

Figure 3.7 Spectral efficiency coverage circles for $n=3.5 \ldots \ldots \ldots \ldots \ldots \ldots \ldots \ldots \ldots$

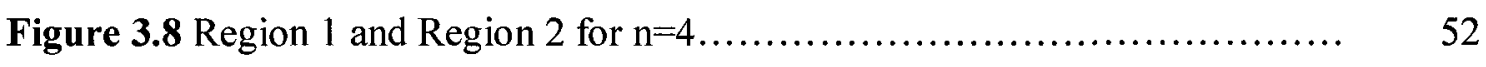

Table 3.4 Position and number of the first tier RSs.......................... 54 


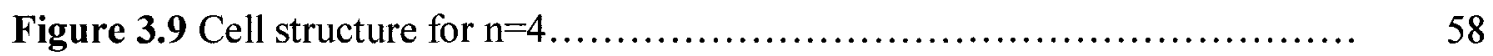

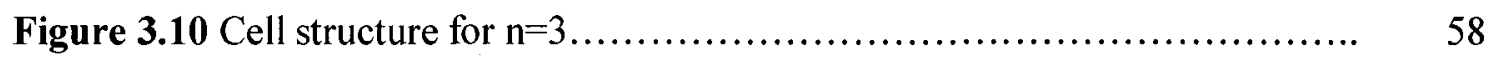

Figure 3.11 Relation between the number of RSs needed and propagation constant

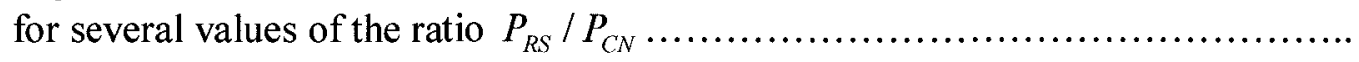

Figure 4.1 Intended signal and interferers for a MS ......................... 69

Figure 4.2 Intended signal and interferers for a MS using relay assistance.......... 71

Figure 4.3 Simulation flowchart ....................................... $\quad 72$

Figure 5.1 Spectral efficiency distribution for non-relaying case $(\mathrm{R}=500 \mathrm{~m}) \ldots \ldots \ldots . \quad 75$

Figure 5.2 Spectral efficiency distribution for $6 \mathrm{RS}$ case $(\mathrm{R}=500 \mathrm{~m}) \ldots \ldots \ldots \ldots \ldots . .75$

Figure 5.3 Spectral efficiency distribution for $18 \mathrm{RS}$ case $(\mathrm{R}=500 \mathrm{~m}) \ldots \ldots \ldots \ldots \ldots . \quad 76$

Figure 5.4 Spectral efficiency distribution for $30 \mathrm{RS}$ case $(\mathrm{R}=500 \mathrm{~m}) \ldots \ldots \ldots \ldots \ldots . \quad 76$

Figure 5.5 Spectral efficiency distribution for $50 \mathrm{RS}$ case $(\mathrm{R}=500 \mathrm{~m}) \ldots \ldots \ldots \ldots \ldots . \quad 77$

Figure 5.6 Spectral efficiency distribution for no RS case $(\mathrm{R}=1000 \mathrm{~m}) \ldots \ldots \ldots \ldots \ldots \ldots . . . \ldots 77$

Figure 5.7 Spectral efficiency distribution for 6 RS case $(\mathrm{R}=1000 \mathrm{~m}) \ldots \ldots \ldots \ldots \ldots \ldots .78$

Figure 5.8 Spectral efficiency distribution for $18 \mathrm{RS}$ case $(\mathrm{R}=1000 \mathrm{~m}) \ldots \ldots \ldots \ldots \ldots . \quad 78$

Figure 5.9 Spectral efficiency distribution for $30 \mathrm{RS}$ case $(\mathrm{R}=1000 \mathrm{~m}) \ldots \ldots \ldots \ldots \ldots . \quad 79$

Figure 5.10 Spectral efficiency distribution for $50 \mathrm{RS}$ case $(\mathrm{R}=1000 \mathrm{~m}) \ldots \ldots \ldots \ldots \ldots$

Figure 5.11 Coverage at various SE levels wrt no. of RSs for $500 \mathrm{~m} \ldots \ldots \ldots \ldots \ldots . . . . . \quad 82$

Figure 5.12 Coverage at various SE levels wrt no. of RSs for $1000 \mathrm{~m} \ldots \ldots \ldots \ldots \ldots . . . . . \quad 82$

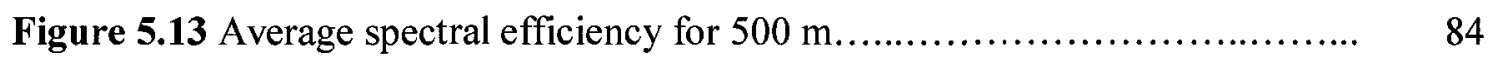

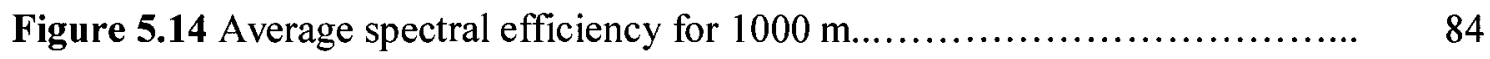

Figure 5.15 Outage for various number of RSs.......................... 85 
Figure 5.16 Coverage at various SE levels wrt RS positions for $500 \mathrm{~m} \ldots \ldots \ldots \ldots \ldots . \quad 87$

Figure 5.17 (Zoomed) Coverage at various SE levels wrt RS positions for $500 \mathrm{~m} . . \quad 87$

Figure 5.18 Coverage at various SE levels wrt RS positions for $1000 \mathrm{~m} \ldots \ldots \ldots \ldots \ldots . \quad 88$

Figure 5.19 (Zoomed) Coverage at various SE levels wrt RS positions for $1000 \mathrm{~m} . \quad 88$

Figure 5.20 Coverage at various SE levels wrt ISF, $500 \mathrm{~m}, 30 \mathrm{RSs} \ldots \ldots \ldots \ldots \ldots \ldots . . \ldots$

Figure 5.21 Coverage at various SE levels wrt ISF, $1000 \mathrm{~m}, 30 \mathrm{RSs} \ldots \ldots \ldots \ldots \ldots . \quad 90$

Figure 5.22 Coverage at various SE levels wrt RS Tx power, 500 m, 30 RSs ...... 91

Figure 5.23 Coverage at various SE levels wrt RS Tx power, $1000 \mathrm{~m}, 30 \mathrm{RSs} \ldots \ldots . \quad 92$

Figure 5.24 Percentage of time using a spectral efficiency, for $500 \mathrm{~m} \ldots \ldots \ldots \ldots \ldots . \quad 93$

Figure 5.25 Percentage of time using a spectral efficiency, for $1000 \mathrm{~m}$............ 94

Figure A.1 Central node and nodes belong to a binary tree $\ldots \ldots \ldots \ldots \ldots \ldots \ldots \ldots, 101$

Table A.1 The percentage of the usage of the spectral efficiency levels .......... 104

Table A.2 The percentage of the usage of the spectral efficiency levels for ideal

2-hop case.

105 


\section{List of Acronyms}

AMC

BER

BICM

BS

$\mathrm{CCN}$

CN

CFRN

MCN

MS

PCN

QAM

QoS

QPSK

RS

SIR

SINR

TDD
Adaptive Modulation and Coding

Bit Error Rate

Bit-Interleaved Coded Modulation

Base Station

Conventional Cellular Networks

Central Node

Cellular Fixed Relay Networks

Micro-Cellular Networks

Mobile Station

Pico-Cellular Networks

Quadrature Amplitude Modulation

Quality of Service

Quadrature Phase Shift Keying

Relay Station

Signal-to-Interference Ratio

Signal-to-Interference-plus-Noise Ratio

Time Division Duplexing 
TDMA

UE

UL

Uplink 


\section{List of Symbols}

$a_{c} \quad$ Combined spectral efficiency for the composite multi-hop link

$a_{i} \quad$ Spectral efficiency of the $i$-th multi-hop link

$a_{\max } \quad$ Maximum attainable spectral efficiency for the system

$b \quad$ Spectral efficiency of the single-hop link

$B \quad$ Bandwidth given for each relay

c Speed of light

C Set of all channel groups available in the cell

$c_{c} \quad$ Combined spectral efficiency for the composite multi-hop link where time slots are reused

$C_{i} \quad$ Set of channel groups available to the $i$-th hop links

$d \quad$ Radius of the relay high data rate coverage area

$d_{0} \quad$ Close-in reference distance

$d_{T R} \quad$ Distance between the transmitter and the receiver antennas

$f \quad$ Carrier frequency

F $\quad$ Frequency reuse factor

$F_{N} \quad$ Noise figure

$G \quad$ Combined antenna gain of the receiver and transmitter 


$\begin{array}{ll}G_{r} & \text { Receiver antenna gain } \\ G_{t} & \text { Transmitter antenna gain } \\ M & \text { Number of "root nodes" in a cell } \\ n & \text { Propagation exponent } \\ N_{i} & \text { Number of nodes in a tree } \\ N_{0} & \text { Number of available channel groups } \\ N_{P C N} & \text { Number of pico-cell base stations in the service area } \\ r_{i} & \text { Number of child "nodes" } \\ P_{B S} & \text { Base Station transmit power } \\ P_{S} & \text { Relay station transmit power } \\ P_{C N} & \text { Central node transmit power } \\ P_{I} & \text { Received interference power } \\ P_{N} & \text { Noise power at the receiver } \\ & \end{array}$




\begin{tabular}{|c|c|}
\hline$R_{B}$ & Capacity of a relay \\
\hline$R_{B S}$ & Capacity of pico-cell base station \\
\hline$R_{C C N}$ & Conventional Cellular Networks cell capacity \\
\hline$R_{C R N}$ & Cellular (Fixed) Relay Networks cell capacity \\
\hline$R_{P C N}$ & Pico-Cellular Networks cell capacity \\
\hline$S E$ & Spectral efficiency in bits/sec/Hz \\
\hline$T_{i}$ & Time in seconds assigned to the $i$-th multi-hop link \\
\hline$T_{M H}$ & Total amount of time assigned to the composite multi-hop link \\
\hline$T_{S H}$ & Total amount of time assigned to the single-hop link \\
\hline$X_{\sigma}$ & Gaussian random variable with standard deviation $\sigma$ \\
\hline$W$ & Available bandwidth per cell in $\mathrm{Hz}$ \\
\hline$Y$ & An exponential random variable representing multipath fading \\
\hline$\gamma^{d B}$ & SINR in $\mathrm{dB}$ \\
\hline
\end{tabular}




\section{CHAPTER 1: INTRODUCTION}

Future wireless systems are expected to provide very high data rates; it is estimated that, for $4 \mathrm{G}$ systems, the aggregate peak data rate demands for mobile access and nomadic/local area wireless access could be around $100 \mathrm{Mbps}$ and $1 \mathrm{Gbps}$, respectively [1]. With the existing cellular architecture, this goal cannot be realized over a wide coverage area. Therefore, some novel cellular network architectures are being studied.

\subsection{Thesis Motivation}

In Conventional Cellular Networks $(\mathrm{CCN})$ each Base Station (BS) is allocated a portion of the available resources (or all of the resources if the frequency reuse factor is 1) and provides service for the users in the cell area. The cell area is determined by the acceptable level of signal-to-noise ratio (SNR) and thus depends on the transmit power and the propagation conditions. The users inside that cell area determine the cell demand. For the same cell, the cell capacity can be found from the available resources to the cell and the QoS requirements of the users in the cell area.

There are two possible problems depending on the cell demand; if the cell demand is greater than the cell capacity, then BS cannot meet the QoS requirements of the users in its cell area. This problem is known as the capacity shortage and can be solved by increasing the number of BSs (maybe along with a decrease in the BS transmit power to 
reduce the interference). Although the solution is not cost effective, since there are demanding subscribers in the cell, the costs can be covered easily. On the other hand, if the cell demand is less than the cell capacity, then a coverage shortage may occur. Even though the cell capacity is enough to meet the cell demand, due to some environmental and propagation conditions, the users in the cell may not get enough SNR to meet their QoS requirements. The cell splitting idea may be used to solve this problem as well; however, in such a case, only a small number of users will be assigned to the newly deployed BSs, and hence the number of the subscribers will not be enough to cover the additional costs. Therefore, a cost effective solution must be found to overcome the coverage shortage problem.

Towards that end, multi-hop relaying concept is considered to be a promising solution [2]. Adding relays in the cells reduces the signal transmission distances, resulting in lower propagation loss and higher average SNR to the mobile user. Therefore, range extension and outage reduction can be obtained by deploying relays [3]. In addition, relays are much simpler devices than base stations in the sense that they require low transmit power and limited functionality [4]; that is, their complexity is comparable to that of WLAN access points. In fact relays are more cost effective due to the fact that, unlike WLAN access points, they do not require a wired backhaul connection. 


\subsection{Research Overview}

This research is on Cellular Fixed Relay Networks (CFRN), a promising cost effective solution to the high data rate coverage problem of the next generation cellular networks. The thesis is composed of two parts; in the first part, the capacity (in terms of aggregate throughput) of CFRN is found and compared to that of Conventional Cellular Networks $(\mathrm{CCN})$ and Pico-Cellular Networks (PCN). In CCN, BSs are deployed in the service area and each BS is responsible for the users in its coverage area. The architecture of PCN is very similar to that of $\mathrm{CCN}$, i.e. each cell has its own BS to provide service to the users in the cell area and all the BSs in the service area are connected to the Internet; however, the main difference is that, the cell sizes are much smaller. On the other hand, the architecture of CFRN used in this part is quite different from that of $\mathrm{CCN}$ and PCN; a large $(\mathrm{CCN})$ cell is divided into smaller cells (same size as PCN cells). Each small cell has a "node" or Relay Station (RS) at the center in order to provide service to the users in its coverage area, as shown in Figure 2.5. In the large cell area, only the Central Node $(\mathrm{CN})$ has a connection to the Internet; in the same large cell, communication between any two nodes is via wireless link. Therefore, besides the amount of bandwidth assigned to the users in the service area, some portion of the available bandwidth should be assigned to the node-to-node links in order to carry the traffic of the users to/from $\mathrm{CN}$.

It is assumed that, each node can directly communicate with only the neighbor nodes, so $\mathrm{CN}$ has direct links with only the first tier nodes. For example, if some data is to be sent from $\mathrm{CN}$ to a user in the coverage region of a third tier node, data must be relayed through one first tier and one second tier node; so, all data (except the amount $\mathrm{CN}$ uses in 
its coverage area) must go through links between the $\mathrm{CN}$ and the first tier nodes. All that data, except for the amount first tier nodes use in their coverage area, should go through the links between first tier nodes and second tier nodes. Due to the difference between the amount of data carried on the $\mathrm{CN}$-(first tier node) links and (first tier node)-(second tier node) links, the amount of bandwidth that should be assigned to the $\mathrm{CN}$-(first tier node) links must be more than the bandwidth that should be assigned to the (first tier node)(second tier node) links. In the same manner, the amount of bandwidth that should be assigned to the (first tier node)-(second tier node) links must be more than the bandwidth that should be assigned to the (second tier node)-(third tier node) links; so, the inner node-to-node links will require more bandwidth than the outer ones.

In the second part of the thesis, optimum number and position of the first tier RSs are found in order to provide high data rate coverage throughout the cell. First, using some analytical calculations, the closest point to the $\mathrm{CN}$ where $n$-hop communication can be utilized is found. Spectral efficiency is used as the data rate comparison criterion. The cell area is composed of regions depending on the spectral efficiency values (or SINR values) of the users. When the effects of shadowing and fading are ignored, then the users having the same spectral efficiency values constitute a circle; so according to the radii of those circles, it is possible to locate the users for which $n$-hop is the best communication type. If, for a user, single-hop spectral efficiency is greater than any kind of multi-hop spectral efficiency, it is better to use single-hop communication for that user. The innermost circle will represent the users for which single-hop gives the best spectral efficiency, so there is no need to use any kind of multi-hop communication. Since multi- 
hop is not needed, putting any RSs in that region would be a waste. Similarly, according to the spectral efficiency circles, the closest distance from the $\mathrm{CN}$ that (in general) $n$-hop communication is practical is found.

It is aimed that the RSs would be simple and small devices compared to Base Stations (BSs), so they would need two separate channels to transmit and to receive. In this analysis, it is assumed that RSs do not transmit and receive at the same time. Therefore, in a composite multi-hop link, time 'set's of two adjacent links are orthogonal to each other. Moreover, in this part, it is also assumed that all other time 'set's for the same composite multi-hop link are also considered as orthogonal in order to reduce the interference in the cell.

For 2-hop communication, each RS needs two orthogonal time slots and operates differently during each of those time slots. When a user or Mobile Station (MS) enters the system, a channel is assigned to that user by the $\mathrm{CN}$. If the user does not need relay assistance, then the operation is same as the CCN case. On the other hand, if relay assistance is needed, then there should be two time slots, one for CN-RS communication, namely $T_{1}$, and one for RS-MS communication, namely $T_{2}$. During $T_{1}$, the channel assigned to the intended MS is used for the CN-RS link and during that time slot the RS operates at receiver only mode. During $T_{2}$, the very same channel is used for the RS-MS link and during that time slot the RS operates at transmitter only mode.

Simulation results support the analytical findings and show that with the proper position and number of RSs, it is possible to increase the SINR values of the MSs 
significantly. Furthermore, additional improvements can be obtained if Adaptive Modulation and Coding (AMC) is used and ubiquitous high data rate can be provided.

\subsection{Thesis Objective}

The main objectives of this research are:

- To investigate whether multi-hop relaying can provide ubiquitous high data rate coverage.

- To analyze the number of RSs needed for various system parameters (such as propagation exponent and relay transmit power).

- To analyze the optimum location of the RSs in the cell.

- To investigate the capacity improvement in terms of aggregate cell throughput when multi-hop is used and to compare the capacity of CFRN to that of CCN and PCN.

- To analyze the multi-hop relaying and to determine the closest point in the cell where $n$-hop communication is practical.

- To investigate the impact of adaptive modulation techniques on the system throughput when relaying is incorporated. 


\subsection{Relevant Literature}

It is known for a long time that relaying is capable of improving the system performance in terms of coverage. The primitive versions of relays are repeaters and they are used to eliminate the black spots and hence to improve the coverage in the cell [5-8]. Those repeaters are "blind" in the sense that they transmit what they receive. In the recent years, due to the improvements in RF technology, the repeaters are evolved to relays (or intelligent relays) and they are no longer "blind", i.e. they transmit the intended parts of the received signal and signal goes through the favorable links. [9-12] present intelligent relaying for the future wireless communications systems. It shown that, with intelligent relaying, the cell coverage and throughput can be improved significantly.

Coverage enhancement in mobile cellular networks through peer-to-peer relaying is studied in $[13,14]$. The results show that relaying improves the system performance significantly, especially when the user density is high. Furthermore, when power control is used, the system becomes more robust to the channel selection scheme.

The theoretical characterizations and analysis for the physical layer of multi-hop wireless communications channels are presented in [15]. It is shown that multi-hop channels outperform single-hop channels. It is also indicated that the performance of analog relaying is better than that of digital relaying for the given system architecture. Furthermore, the usage of diversity improves the performance of multi-hop channels for both analog and digital relaying. 
The range extension feature of the relaying concept can be applied to the HiperLAN/2 type of networks as well [16-18]. A possible deployment scenario is introduced and the simulative traffic performance in a Manhattan-like dense urban environment, where too much capacity is provided for a small area, is presented. It is shown that infrastructure based relaying can be used to trade capacity against range with an increase of the peak throughput. Additionally, the required transmit power can be significantly reduced compared to a single-hop system.

One other aspect of relaying is load balancing, which is described in [19]. The main idea is to divert the traffic of a highly loaded (congested) cell to the adjacent cells, preferably non-congested ones (or the ones having available data channels), using the relays. It is shown that, relaying can enhance the coverage and, with load balancing, provide a higher throughput.

\subsection{Thesis Organization}

This thesis is structured as follows: Chapter 2 studies the capacity of CFRN. The system architecture describes the node locations, antenna types, and traffic route for the system. Then, capacity calculations for the given CFRN structure are presented. To simplify the analysis, a simple frequency assignment scheme is used for the nodes. Later in the same chapter, the frequency assignment issue is generalized and capacity calculations are done for the generalized case. Finally, capacity calculations of $\mathrm{CCN}$ and PCN are done and compared to that of CFRN. 
Chapter 3 describes the system model for CFRN in order to find the optimized number and position of RSs. Time slot assignment for composite multi-hop link is explained. Then, the data rate ratio of multi-hop communication and single-hop communication is found in terms of spectral efficiency values. The spectral efficiency coverage circle issue is explained and radii of the circles are found. With some calculations, the optimum position and number of the first tier RSs are found. Finally, the reuse of time slots is discussed.

Chapter 4 presents the simulation model. Propagation model and environmental parameters are introduced. The simulation procedure is then briefly explained followed by flow chart of the main simulation.

Chapter 5 discusses the simulation results. Simulation results are categorized into three main sections: spectral efficiency distribution and coverage, outage probability, and adaptive modulation and coding histograms. Results for single-hop and 2-hop cases are compared for various RS positions and numbers and discussions are presented.

Chapter 6 provides the conclusions from this work. The contributions of the thesis are discussed and some suggestions are made on possible future research in this area. 


\section{CHAPTER 2: CAPACITY OF CELLULAR FIXED RELAY NETWORKS}

The Cellular Fixed Relay Networks (CFRN) is a generalized form of the relaying concept. The whole cell area is covered by large number of Relay Stations (RSs), i.e. each RS serves a small area with a small amount of power. Due to the low power feature, when there is sufficient electromagnetic separation, it is possible to reuse the channels even in the same cell.

In this chapter, the first section introduces the architecture of the CFRN. Then the cell capacity, i.e. aggregate throughput, of the CFRN is found, enabling the comparison between the capacities of the CFRN and Conventional Cellular Networks (CCN). Finally, the capacity of Pico-Cellular Networks (PCN) is found and compared to that of CFRN.

\subsection{System Architecture}

Figure 2.1 shows a possible tree structure for Cellular Fixed Relay Networks (CFRN). The solid lines in the figure represent the wireless links. Each dot denotes a node or equivalently a relay; in this research, these two terms (node and relay) are used interchangeably. As seen from the figure, CFRN may be considered as a combination of a central node (BS or $\mathrm{CN}$ ) and several trees, whose nodes are fixed relays; without loss of generalization, it is assumed that all trees are binary trees, i.e. each node has one parent 
node and two child nodes ${ }^{1}$; other cases are also considered later in the flowing sections. Although, in Figure 2.1, the roots of all trees are positioned on a ring around the central node, such regular geometric patterns are not required.

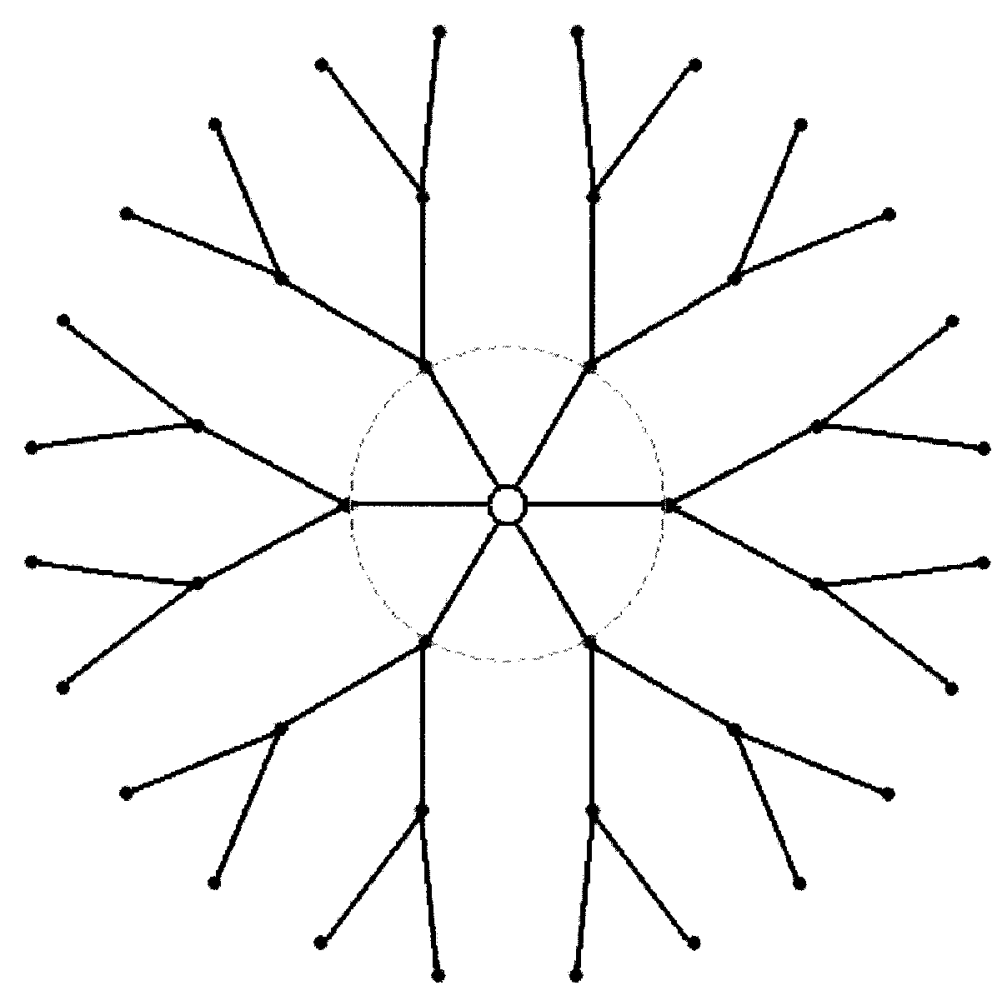

Figure 2.1: CFRN configuration.

All of the nodes are placed at positions where they have good links with their parent and child nodes. Each node has two different types of antenna:

- Omni-directional (or sectorized) antenna

- Highly directional narrow beam antenna

${ }^{1}$ Details are given in Appendix A. 
Each node uses the omni-directional (or sectorized) antenna to communicate with the users in its coverage area. This communication is similar to one between a Base Station with omni-directional (or sectorized) antenna and the users in the cell. In addition to this, nodes have highly directional narrow beam antennas (such as pencil-beam antennas), for point-to-point communication; each node has three beam antennas: two for its child nodes and one for its parent node, with the exception of the leaf node (relay on the cell boundary) having only one for its parent node.

The central node, unlike the relay nodes, has a connection to the wired network. It has as many directional antennas as the number of the root nodes. This reduces the interference among primary links emanating from the central node. This feature enables the simultaneous use of the very same frequency band in all those links. Similar to relays, the central node uses an omni-directional antenna to communicate with the users in its coverage area.

Each node is responsible for the traffic in its coverage area and the traffic that needs to be relayed through it, i.e. traffic for the sub-tree originating from itself. For the downlink, each node gets traffic of its own and traffic that has to be relayed to its child nodes from its parent node. For the uplink, each node gets traffic from its child nodes and relays it to its parent node along with its own traffic.

Since the cell area is covered by a large number of relays, there is no need for a high power central node. Actually, if the number of relays is high enough, it may be assumed that all relays and the central node have approximately the same coverage area. Therefore, transmit power of the central node can be reduced to that of a relay. Since 
coverage areas of central node and the relays are considered to be equal, available bandwidth for central node and other nodes can be taken as equal as well. Although the features of a central node and a relay are almost the same, there is one fundamental difference: the central node has a connection to the wired network, whereas relays have no wired access to Internet.

\subsection{Capacity of the CFRN}

In this work, the term "capacity" refers to the aggregate throughput.

The central node has a direct link with all of the root nodes. Since all of those links use separate directional antennas, to obtain the maximum capacity, the same bandwidth can be used for all of them. Therefore, if the capacity of one link can be found, that of other links would also be the same. Figure 2.2 shows the central node and nodes that belong to one of the trees.

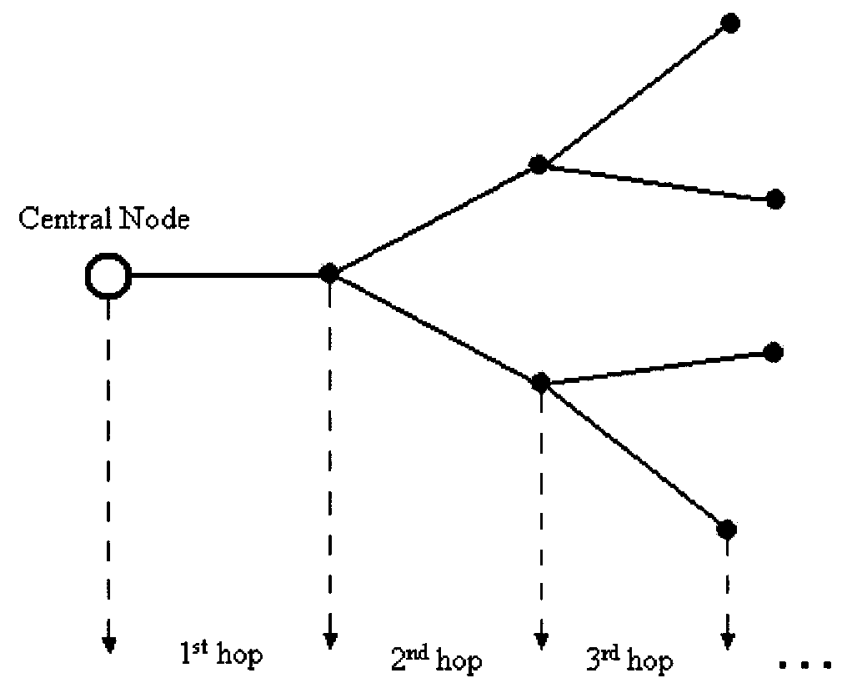

Figure 2.2: Central Node and a tree in a binary tree structure. 
The traffic of all nodes (relays) that belong to the same tree must pass through the link between the central node and the root node, namely the primary link of that tree. Therefore, the capacity of a tree is limited by the capacity of the corresponding primary link.

Let $W$ be the total available bandwidth per cell in $\mathrm{Hz}$, and let $R$ be the capacity per cell in bits/sec. We will denote the cell capacity of the conventional cellular network, $\mathrm{CCN}$ (which does not employ any relays), as $R_{C C N}=R_{W}$ bits $/ \mathrm{sec}$, where $R_{W}$ indicates that the capacity is a function of $W$.

Next, consider a CFRN cell with $M$ root nodes, each of which is responsible for $N-1$ nodes in a binary tree structure (Fig. 2.2). We assume that $B \mathrm{~Hz}$ of bandwidth is required to support the capacity of one relay, which is denoted as $R_{B}$. If the capacity of the central node (BS) is also equal to that of a relay, then

$$
R_{C R N}=(M N+1) R_{B} \approx M N R_{B} .
$$

In order to compare $R_{C R N}$ with $R_{C C N}, R_{B}$ has to be found in terms of $R_{W}$, or equivalently $B$ has to be found in terms of $W$. As previously stated, the total bandwidth, $W$, is used in each tree and is not equal to the bandwidth assigned to a primary link. This is due to the fact that some portion of the total bandwidth is assigned for relay-to-relay communication.

Assume that the total available bandwidth $W$ is divided into channel groups of $B \mathrm{~Hz}$. Figure 2.3 shows the available frequency spectrum. As stated before, each relay uses an 
average bandwidth of $B \mathrm{~Hz}$; this corresponds to one of the channel groups from the set $\left\{1,2,3, \ldots, N_{0}\right\}$ in its coverage area.

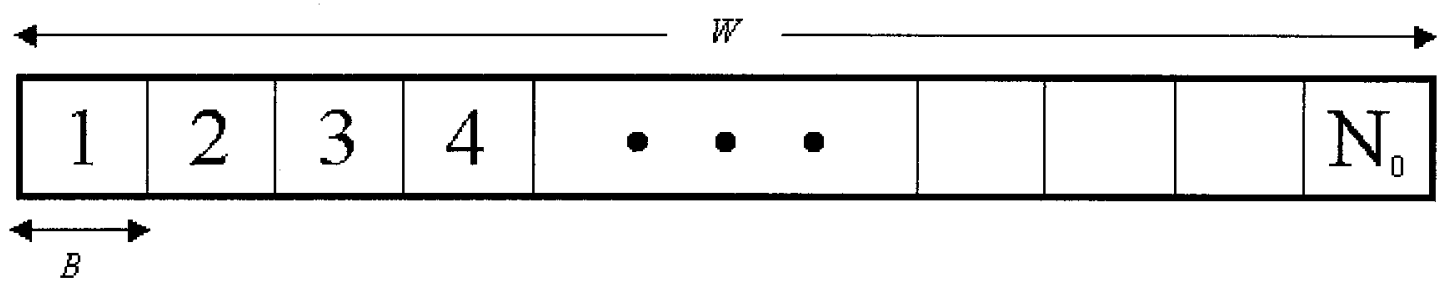

Figure 2.3: Available frequency spectrum.

Let us consider one tree. We define the following sets in a $k$-hop network:

$C:\{$ set of all channel groups available in the cell $\}$

$C_{0}:\{$ set of channel groups used by nodes in their coverage area\}

$C_{i}:\left\{\right.$ set of channel groups used by $i^{\text {th }}$ hop links $\}, i=1,2, \ldots, k$

Note that,

$$
C=C_{0} \cup C_{1} \cup C_{2} \cup \ldots \cup C_{k}
$$

and

$$
s(\boldsymbol{C})=W / B=N_{0} .
$$

In (2.3), $s(\boldsymbol{C})$ stands for the size (cardinality) of the set $C$; also, without loss of generality, we assumed that $W / B$ is an integer. To simplify the calculations, assume for the time being that all nodes (including the central node) use the same channel group, say channel group \#1, from Figure 2.3, in their coverage area. This is similar to the case 
where nodes are considered as pico-cell base stations, each of which uses channel group $\# 1$ to communicate with the users in its coverage area, regardless of the interference. It is worth mentioning that this condition will be removed later. Then,

$$
s\left(C_{0}\right)=1 \text { channel group. }
$$

In order not to generate excessive interference, the channel group in set $\boldsymbol{C}_{0}$, channel group \#1, cannot be used by any node-to-node link, including the primary links; therefore,

$$
s\left(C_{0} \cap C_{i}\right)=0, \quad \text { for } i=1,2, \ldots, k \text {. }
$$

The primary link carries $N$ relays' traffic, so, bandwidth of $(N B)$ should be assigned to that link. Thus,

$$
s\left(\boldsymbol{C}_{\boldsymbol{I}}\right)=N \text { channels groups. }
$$

Due to (2.5), channel group \#1 cannot be used for primary link, then, channel groups from $\# 2$ to $\#(N+1)$ in Figure 2.3 should be assigned for the primary link.

Each second hop link is responsible for the traffic of $\left\lceil\frac{N-1}{2}\right\rceil$ nodes, so each second hop link needs $\left\lceil\frac{N-1}{2}\right\rceil$ channels. Since each node has 3 different directional antennas (one for its parent node, two for its child nodes), both antennas directed to the child nodes 
can use the same channel groups; therefore, for the second hop links, a total of $\left\lceil\frac{N-1}{2}\right\rceil$ channel groups are needed:

$$
s\left(C_{2}\right)=\left\lceil\frac{N-1}{2}\right\rceil \text { channel groups. }
$$

Assuming that relays cannot transmit and receive at the same frequency at the same time, the second hop link cannot use the $N$ channel groups used by the primary link. Therefore,

$$
s\left(C_{1} \cap C_{2}\right)=0
$$

Due to $(2.5) \&(2.8)$, channel groups from $\# 1$ to $\#(\mathrm{~N}+1)$ in Figure 2.3 cannot be assigned to the second hop link; so starting from channel group \#(N+2), $\left\lceil\frac{N-1}{2}\right\rceil$ channel groups should be assigned to this link.

Each third hop link carries the traffic of $\left\lceil\frac{N-3}{4}\right\rceil$ relays. As for the second hop links, each third hop link can use the same set of channel groups; therefore for the third hop links, a total of $\left\lceil\frac{N-3}{4}\right\rceil$ channel groups are required. Once again, since relays cannot transmit and receive on the same channel, none of the channel groups used in the second hop links can be assigned to third hop links. Instead, for the third hop links, a subset of the primary link channel groups can be used (since the primary links do not create 
interference on third hop links and they require fewer channel groups than the primary links), i.e. $\left\lceil\frac{N-3}{4}\right\rceil$ channel groups from $\# 2$ to $\#(\mathrm{~N}+1)$. In the same manner, for the $4^{\text {th }}$ hop links, a subset of the second hop channel groups can be used. So, in general,

$$
C_{1} \supset C_{2 j-1} \text { and } C_{2} \supset C_{2 j} \text { for } j=2,3, \ldots
$$

Then, from (2.9)

$$
\begin{aligned}
& s\left(C_{1} \cup C_{2 j-1}\right)=s\left(C_{1}\right), \\
& s\left(C_{2} \cup C_{2 j}\right)=s\left(C_{2}\right) .
\end{aligned}
$$

The total number of channel groups can be found from (2.2) and (2.10) as,

$$
\begin{aligned}
s(C)= & s\left[C_{0} \cup\left(C_{1} \cup \ldots \cup C_{2 j-1} \cup \ldots\right)\right. \\
& \left.\cup\left(C_{2} \cup \ldots \cup C_{2 j} \cup \ldots\right)\right], j=2,3, \ldots, k \\
= & s\left(C_{0} \cup C_{1} \cup C_{2}\right),
\end{aligned}
$$

which can further be simplified by using (2.5) and (2.8);

$$
s(C)=s\left(C_{0}\right)+s\left(C_{1}\right)+s\left(C_{2}\right) .
$$

Now, from (2.4), (2.6), and (2.7)

$$
N_{0}=N+\left\lceil\frac{N-1}{2}\right\rceil+1 \text { channel groups. }
$$


Figure 2.4 shows an example of a tree with 7 nodes to find the total number of channel groups. As stated before, it is assumed that all the nodes use the same channel group, \#1 from Figure 2.3, in their coverage area. Seven additional channel groups are needed for the primary link as there are seven nodes in the tree; therefore channel groups \#2 - \#8 are assigned to this link. Second hop links are responsible for the traffic of 3 nodes, so these links need 3 channel groups. However, those 3 channel groups must be different than the ones used for the primary link due of the fact that relays cannot transmit and receive on the same channel. Then, channel groups $\# 9$ - \#11 are assigned for the two second hop links; both second hop links have separate directional antennas, enabling the reuse of the same channel groups. Each of the third hop links carries the traffic of only one relay, so only one channel group is required. This channel group shouldn't be the same as one of the channel groups used for second hop links, whereas, one of the channel groups used for the primary link can be used (in Figure 2.4 channel group \#2 is used). To sum up, as a total of 11 channel groups are used for this tree. 


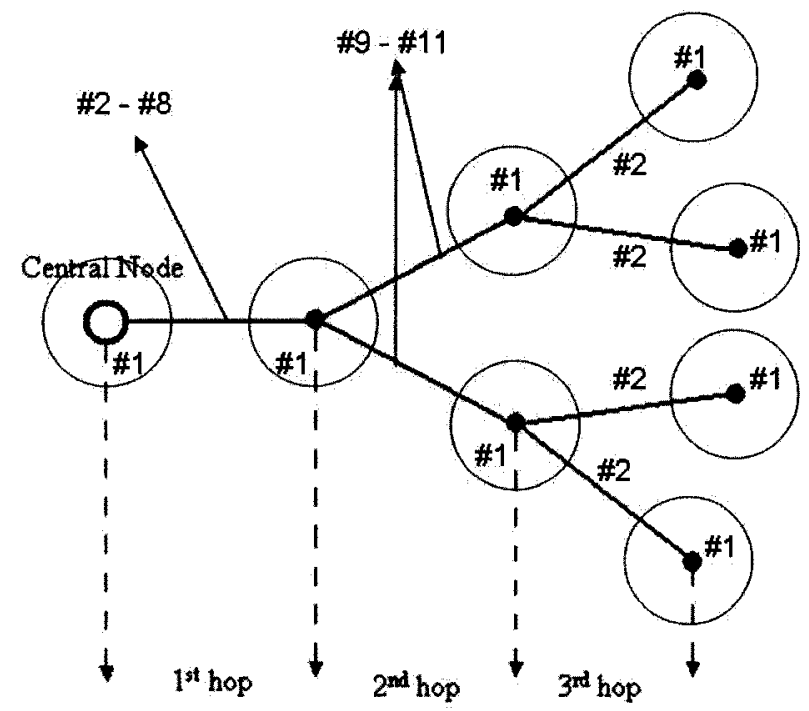

Figure 2.4: Assigned channel groups for a tree of 7 nodes.

Finally, from (2.3) the relation between $W$ and $B$ can be obtained as,

$$
W=\left\{N+\left\lceil\frac{N-1}{2}\right\rceil+1\right\} B .
$$

Due to the one to one correspondence between bandwidth and capacity, the relation between $R_{W}$ and $R_{B}$ can be obtained from (2.13) as,

$$
R_{W}=\left\{N+\left\lceil\frac{N-1}{2}\right\rceil+1\right\} R_{B} .
$$

At this point we are ready to find $R_{C R N}$ from (2.1) and (2.14) as

$$
R_{C R N} \approx\left(\frac{N}{N+\left\lceil\frac{N-1}{2}\right\rceil+1}\right)\left(M R_{W}\right) .
$$


For large values of $N$, equation (2.15) can be approximated by taking its limit:

$$
\begin{aligned}
R_{C R N} \approx & \left(\lim _{N \rightarrow \infty} \frac{N}{N+\left[\frac{N-1}{2}\right]+1}\right)\left(M R_{W}\right) \\
& \Rightarrow R_{C R N} \approx \frac{2}{3}\left(M R_{W}\right) .
\end{aligned}
$$

On the discussion so far, the corresponding capacity result given in (2.16), has been for the networks with binary trees. In general, if each root node has $p$ child nodes, then ${ }^{2}$

$$
R_{C R N} \approx \frac{p}{p+1}\left(M R_{W}\right)
$$

Equation (2.17) can be interpreted as follows: cell capacity of CFRN is directly proportional to the number of the root nodes; if this number is increased, then the capacity of the cell would increase and accordingly either the number of the users in the cell or the amount of the bandwidth for a user would increase. However, there is a limit on the maximum number of root nodes. In order to get the maximum aggregate cell capacity, all the primary links should use the same bandwidth at the same time. To facilitate such a dense reuse, the central node has to use a separate directional antenna when communicating with each root node. Therefore, the maximum capacity of the CFRN depends on the directivity of the beam antennas used, the number of the nodes in each tree, and the amount of average bandwidth used by each node.

${ }^{2}$ Details are given in the Appendix B. 


\subsection{Generalization of the Discussion}

It has been assumed so far, for simplicity of the analysis, that all the nodes use the same channel group in their coverage area. This can clearly create significant interference. To avoid that, frequency reuse, similar to the one for conventional cellular structure, can be used. Figure 2.5 shows an example frequency reuse pattern for the relay network.

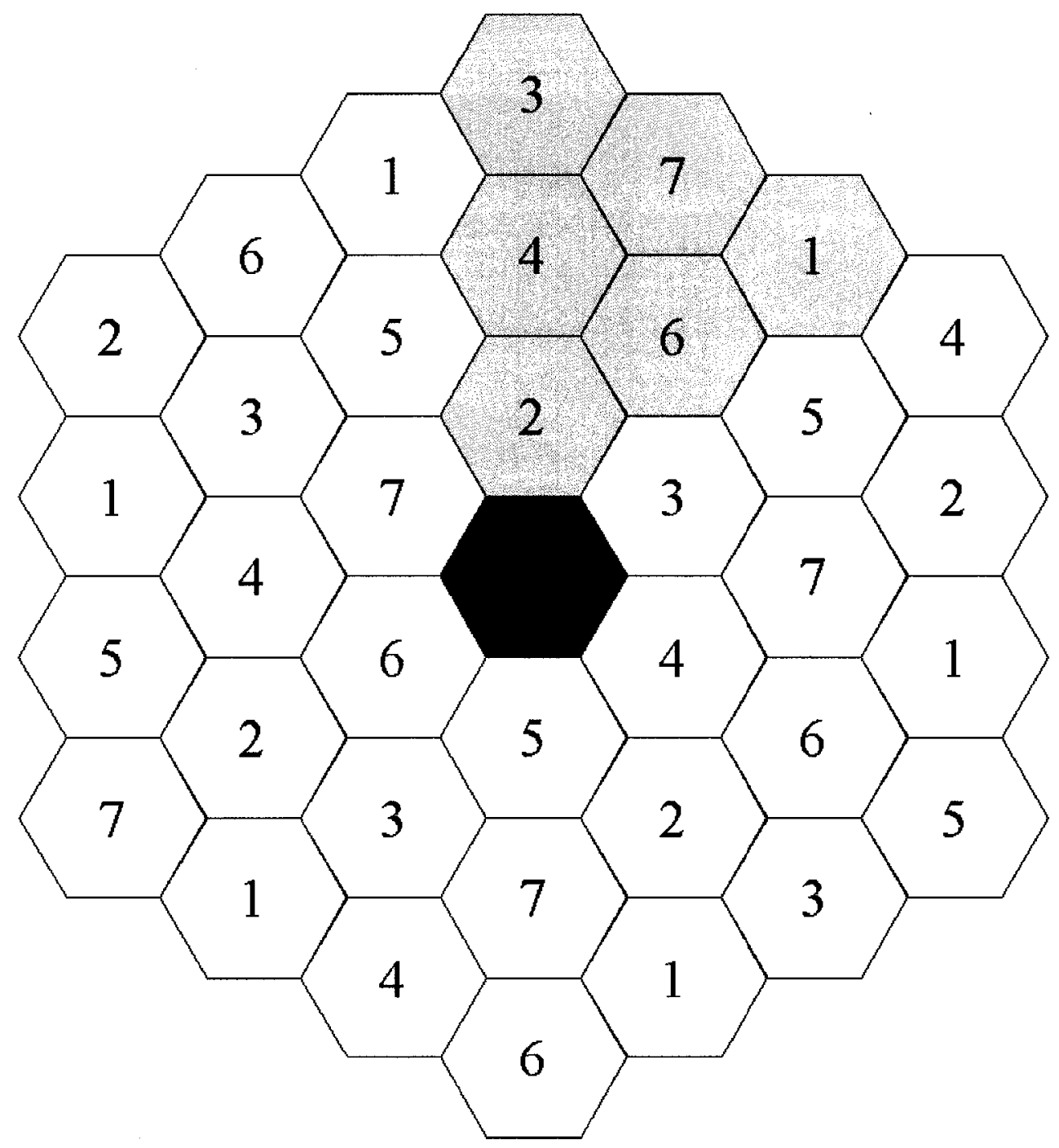

Figure 2.5: General structure of the CFRN. 
In Figure 2.5 the red node is the Central Node and the blue nodes constitute a tree. A frequency reuse factor of 7 is assumed; provided by the numbers in the hexagons represent the channel groups used for the coverage provided by the nodes (e.g. node-toMS links). In order to find the total capacity of this cell, in addition to the channel groups used for coverage areas, channel groups used for node-to-node links must be known as well. Figure 2.6 illustrates an example of the assigned channel groups for the central node and for one of the trees in the cell.

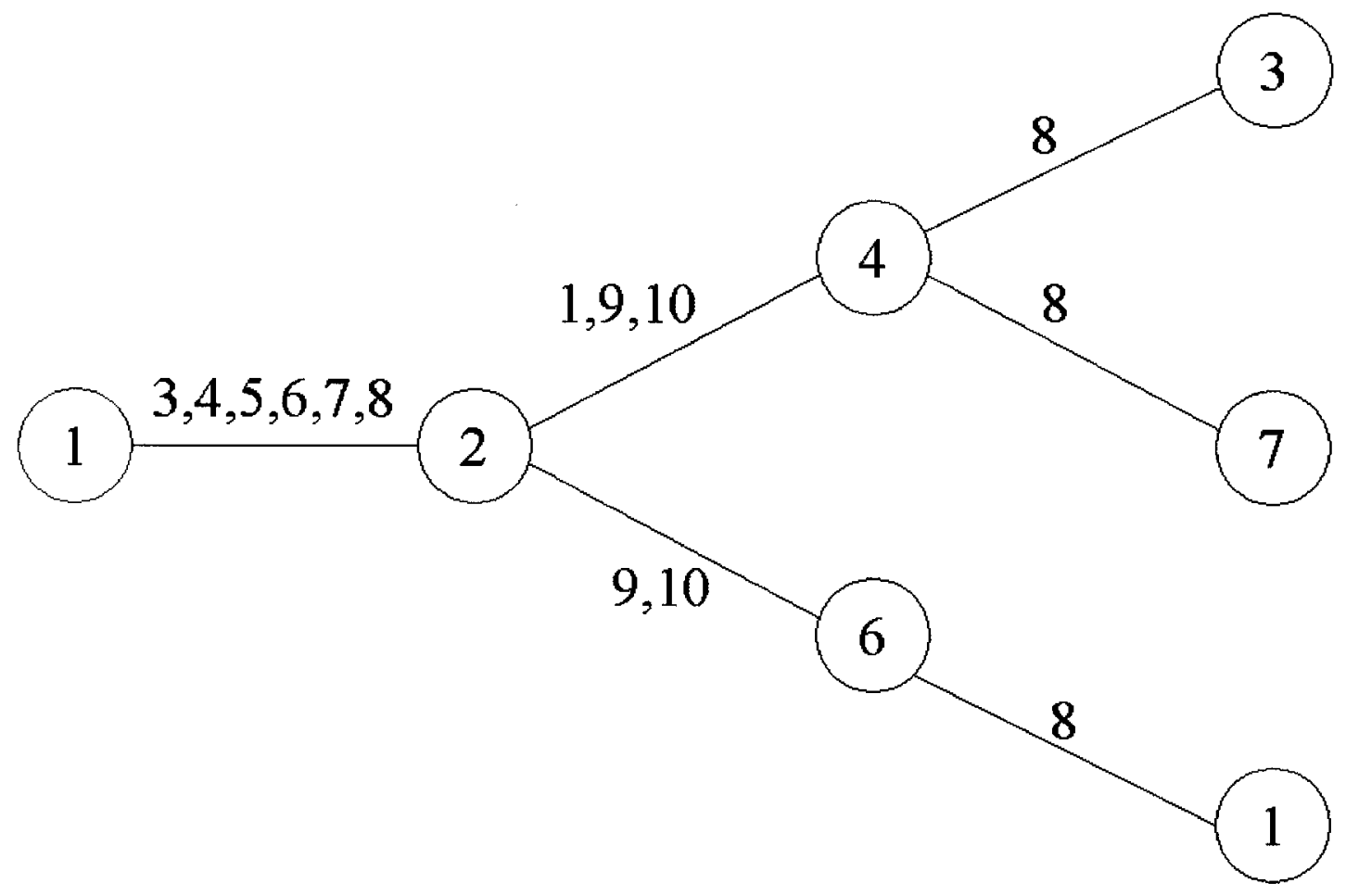

Figure 2.6: Assigned channel group groups for a tree. 
In Figure 2.6 the red circle and the black circles represent the Central Node and the nodes which belong to the tree, respectively. As mentioned before, the numbers inside the circles are the channel groups used in the coverage area of the corresponding nodes. Channel groups for the node-to-node links are assigned as follows:

- The tree in Figure 2.6 has six nodes, so the primary link (i.e. the link between the Central Node and the root node) should carry the traffic of those six nodes. Channel groups \#1 and \#2 are not assigned to this link in order not to create excessive interference, as the Central Node and the root node use those channel groups. Then, the following six channel groups (\#3 - \#8) are assigned to this link.

- The link between the root node and the node that uses channel group \#4 in its coverage area is responsible for carrying the traffic of three nodes, so it needs three channel groups. Channel groups \#2 and \#4 are not assigned for interference reduction, and channel groups \#3 - \#8 are not assigned due to the fact that nodes cannot transmit and receive at the same channel. As a result, the following three channel groups are assigned to this link: \#1, \#9, and \#10.

- The link between the root node and the node that uses channel group \#6 in its coverage area is responsible for carrying the traffic of two nodes, so it needs two channel groups. Since nodes have separate narrow beam antennas for the separate links, this link does not require any additional channel group and can use the channel groups \#9 and \#10. 
- Each link that connects the leaf node to its parent node is responsible from the traffic of the leaf node only, so it needs one channel group. This link can use a channel group from the channel group set used in the primary link. So channel group \#8 is assigned to this link.

Now that all the channel groups are assigned to the given tree, the capacity of the CFRN can be calculated. As seen from Figure 2.6, total number of channel groups used is 10. It is given that the bandwidth available to the system is $W \mathrm{~Hz}$ and the bandwidth of the each channel group used for the coverage area of a node is $B \mathrm{~Hz}$. Then, $W / B$, or $R_{W} / R_{B}$, is equal to 10. Therefore,

$$
\begin{aligned}
R_{C R N} & =(M . N+1) R_{B}=37 R_{B}=3.7 R_{W} \\
& =3.7 R_{C C N} .
\end{aligned}
$$

\subsection{Capacity Comparison of CFRN and Pico-Cellular Networks (PCN)}

Figure 2.5 can be used to compare the capacities of the CFRN and PCN. For a fair comparison the capacity over the same area should be considered. Let's assume that total area of consideration is the area in Figure 2.5. Each hexagon represents the coverage area of a node in the case of CFRN and that of a pico-cellular Base Station in the case of PCN. In this area, the capacity of a CFRN has already been found to be $3.7 R_{W}$. Since all the base stations in PCN are connected to the wired network, the capacity is equal to the number of base stations times the capacity of a base station in the area. That is, 


$$
R_{P C N}=N_{P C N} R_{B S}
$$

where $R_{P C N}$ is the capacity of PCN for the given area, $N_{P C N}$ is the number of pico-cell base stations in the given area, and $R_{B S}$ is the capacity of a pico-cell base station.

The capacity of a pico-cell base station depends on the frequency reuse factor, $F$. If $F$ is equal to 1 (i.e. all of the available bandwidth is used in the coverage area of a base station), then the capacity of a base station is equal to $R_{W}$. In general, each base station uses only $1 / F$ of the available bandwidth in its coverage area. Therefore, the capacity of a base station becomes $R_{W} / F$. Then,

$$
R_{P C N}=N_{P C N} \frac{1}{F} R_{W}
$$

In Figure 2.5, the frequency reuse factor is 7 , so the capacity of the PCN for the given area is:

$$
R_{P C N}=\frac{37}{7} R_{W} \approx 5.3 R_{W}
$$

Equation (2.20) states that, the capacity of the PCN is directly proportional to the number of cells (or nodes) in the given area, and inversely proportional to the frequency reuse factor. For the example in Figure 2.5, the capacity of the CFRN is comparable to that of $\mathrm{PCN}\left(3.7 R_{W}\right.$ vs. $\left.5.3 R_{W}\right)$. However, this is not the case in general. If the frequency reuse factor for both CFRN and PCN is equal to 1, the capacity of CFRN does not 
change; it is equal to $3.7 R_{W}$. On the other hand, the capacity of PCN becomes $37 R_{W}$, which is 10 times the capacity of CFRN.

\subsection{Generalization of (2.17) for Orthogonal Channels}

Equation (2.17) is obtained under the assumption that all the node-to-node links use separate narrow beam antennas so that every other hop links, e.g. first and third hop links, do not create interference for each other; enabling the reuse of the same channel groups for both of these links. Another approach is to assume all the hop links are orthogonal to each, which reduces the interference in the cell. For the analysis following definitions are used,

$N$ : Total number of nodes in one tree

$C_{k}:$ Total number of channel groups for the $k$-th level links

$N_{T}$ : Total number of channel groups needed for a tree

$p:$ Number of child nodes

For a full tree, the number of nodes at level ' $k$ ' is equal to $p^{k-1}$, where level ' 1 ' corresponds to the root node of the tree. Then, total number of nodes in the tree becomes $\left(p^{q}-1\right)$, where $q$ is the maximum value for the level (or depth) of the tree. If we assume that all of the same level links can use the same channel groups, total number of channel groups needed for level $k$ becomes 


$$
C_{k}=\frac{N-\sum_{i=0}^{k-2} p^{i}}{p^{k-1}}=\frac{N-\left(\frac{p^{k-1}-1}{p-1}\right)}{p^{k-1}} .
$$

Since all the hop links use orthogonal channel groups, the intersection of any two hop links must be zero; then the total number of channel groups for a tree can be written as follows,

$$
N_{T}=N+\sum_{k=2}^{q}\left(\frac{N-\left(\frac{p^{k-1}-1}{p-1}\right)}{p^{k-1}}\right)+1=N+N \sum_{k=2}^{q} \frac{1}{p^{k-1}}-\sum_{k=2}^{q} \frac{p^{k-1}-1}{p^{k-1}(p-1)}+1 .
$$

The total number of channel groups in (2.15) can be replaced by (2.23),

$$
R_{C R N} \approx\left(\frac{N}{N+N \sum_{k=2}^{q} \frac{1}{p^{k-1}}-\sum_{k=2}^{q} \frac{p^{k-1}-1}{p^{k-1}(p-1)}+1}\right)\left(M R_{W}\right)
$$

For large number of nodes in the tree,

$$
\begin{aligned}
R_{C R N} & \approx\left[\lim _{N \rightarrow \infty}\left(\frac{N}{N+N \sum_{k=2}^{q} \frac{1}{p^{k-1}}-\sum_{k=2}^{q} \frac{p^{k-1}-1}{p^{k-1}(p-1)}+1}\right)\right]\left(M R_{W}\right) \\
& =\left[\lim _{N \rightarrow \infty}\left(\frac{1}{1+\sum_{k=2}^{q} \frac{1}{p^{k-1}}-\frac{1}{N} \sum_{k=2}^{q} \frac{p^{k-1}-1}{p^{k-1}(p-1)}+\frac{1}{N}}\right)\right]\left(M R_{W}\right) .
\end{aligned}
$$


We can make the following simplifications in the terms in the denominator of the last expression of (2.25):

$$
\begin{aligned}
& \lim _{N \rightarrow \infty}\left(\sum_{k=2}^{q} \frac{1}{p^{k-1}}\right)=\lim _{N \rightarrow \infty}\left(\frac{1-\frac{1}{p^{q-1}}}{p-1}\right)=\lim _{N \rightarrow \infty}\left(\frac{1}{p-1}\right)-\lim _{N \rightarrow \infty}\left(\frac{1}{p^{q-1}(p-1)}\right) \\
& =\left(\frac{1}{p-1}\right)-\lim _{N \rightarrow \infty}\left(\frac{p}{(N+1)(p-1)}\right)=\left(\frac{1}{p-1}\right), \\
& \lim _{N \rightarrow \infty}\left(\frac{1}{N} \sum_{k=2}^{q} \frac{p^{k-1}-1}{p^{k-1}(p-1)}\right)=\lim _{N \rightarrow \infty}\left(\frac{1}{N} \sum_{k=2}^{q} \frac{1}{(p-1)}\right)-\lim _{N \rightarrow \infty}\left(\frac{1}{N} \sum_{k=2}^{q} \frac{1}{p^{k-1}(p-1)}\right) \\
& =\frac{1}{(p-1)} \lim _{N \rightarrow \infty}\left(\frac{q-1}{N}\right)-\frac{1}{(p-1)} \lim _{N \rightarrow \infty}\left(\frac{1}{N(p-1)}-\frac{p}{N(N+1)(p-1)}\right) \\
& =\frac{1}{(p-1)} \lim _{N \rightarrow \infty}\left(\frac{\log _{p}(N+1)}{N}-\frac{1}{N}\right)-\frac{1}{(p-1)} \lim _{N \rightarrow \infty}\left(\frac{1}{N(p-1)}-\frac{p}{N(N+1)(p-1)}\right)=0 \text {, } \\
& \lim _{N \rightarrow \infty}\left(\frac{1}{N}\right)=0
\end{aligned}
$$

Then substituting (2.26)-(2.28) in (2.25), $R_{C R N}$ can be obtained as

$$
R_{C R N}=\frac{p-1}{p} M R_{W}
$$




\section{CHAPTER 3: $\quad$ FEASIBILITY OF PROVIDING HIGH DATA RATE COVERAGE IN CELLULAR FIXED RELAY NETWORKS}

In this part the feasibility of providing high data rate coverage through fixed relay stations (RSs) in cellular networks is studied. Spectral efficiency is used as the performance metric. The main idea behind this is that the data rate is directly proportional to the spectral efficiency. Therefore, maximum throughput is obtained if all MSs in the system can be provided with the maximum available spectral efficiency. It is shown that high data rate coverage is obtained by relatively high number of RSs; on the contrary high number of hops is not required since that results in loss of available channels.

\subsection{System Model}

In a cellular network topology, connections between nodes can be established as either single-hop or multi-hop links. In this context, a hop is considered as the direct wireless connection (or link) between two nodes. This implies that, for a multi-hop communication between two nodes, some number of intermediate nodes are needed to relay the data to be sent from source node to destination node. Figure 3.1 shows the nodes and links for the multi-hop communication between the Central Node $(\mathrm{CN})$ and the Mobile Station (MS) in a cell. In the figure, "square" node represents the $\mathrm{CN}$, equivalent to a base station in Conventional Cellular Networks $(\mathrm{CCN})$, and has a connection to the 
wired network. The "triangle" node represents the MS and each "circle" node represents a Relay Station (RS).

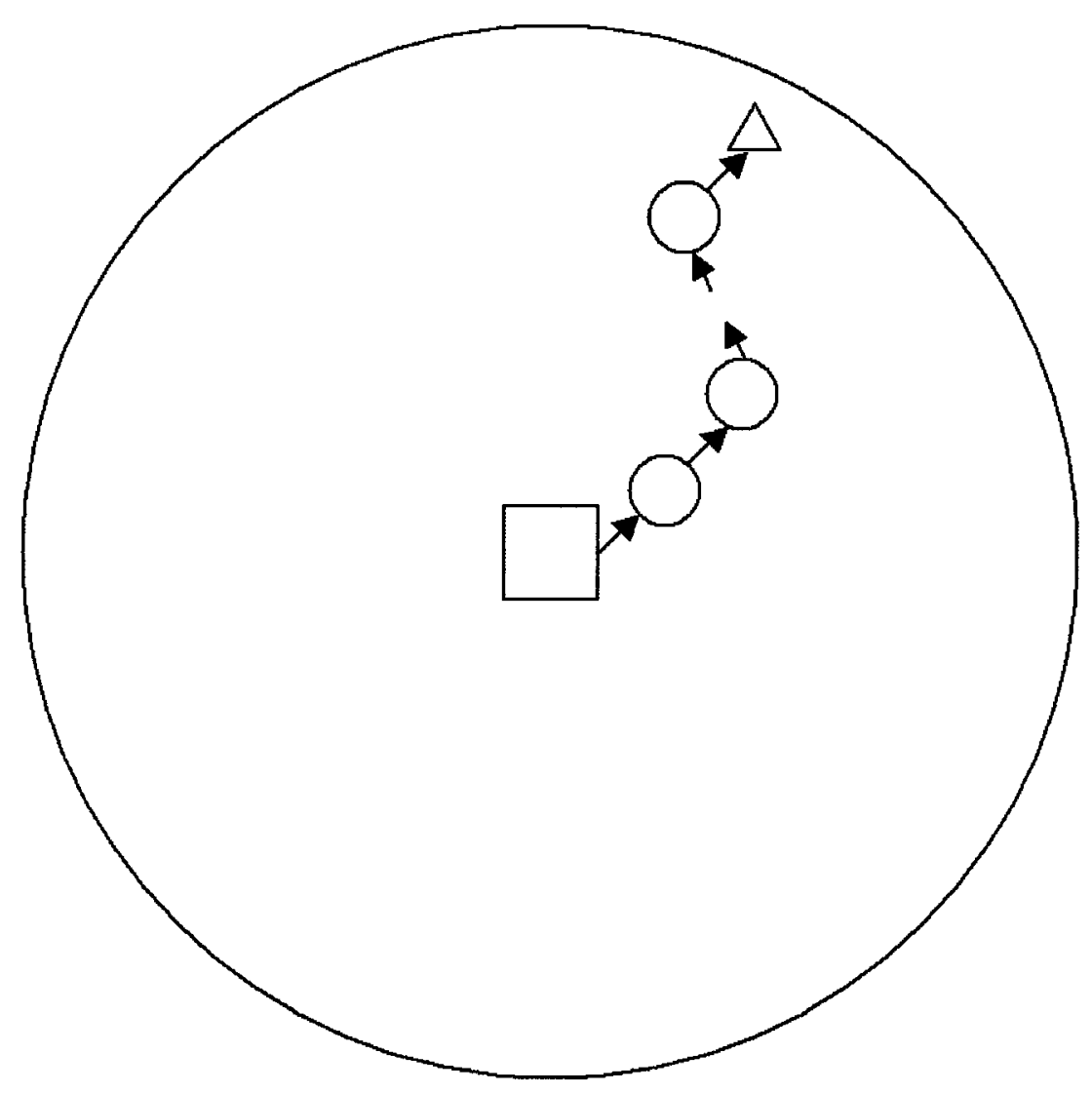

Figure 3.1: $n$-hop communication system architecture.

As seen from Figure 3.1, the composite multi-hop link from $\mathrm{CN}$ to MS is a combination of several short links. Figure 3.2 shows the topology of a composite multihop link. All the links between CN, RSs, and MSs are wireless. Spectral efficiency values (in bits/sec/Hz) for the composite multi-hop link that connects CN to MS are denoted by $a_{1}$ to $a_{n}$. Similarly, spectral efficiency of the single-hop link between the $\mathrm{CN}$ and the same MS is denoted by $b$. Each spectral efficiency is determined from the signal-tointerference-plus-noise ratio (SINR) of the corresponding link. Although the issue of 
signalling overhead is not considered in this research, its effect can be assumed to be included in the spectral efficiency.

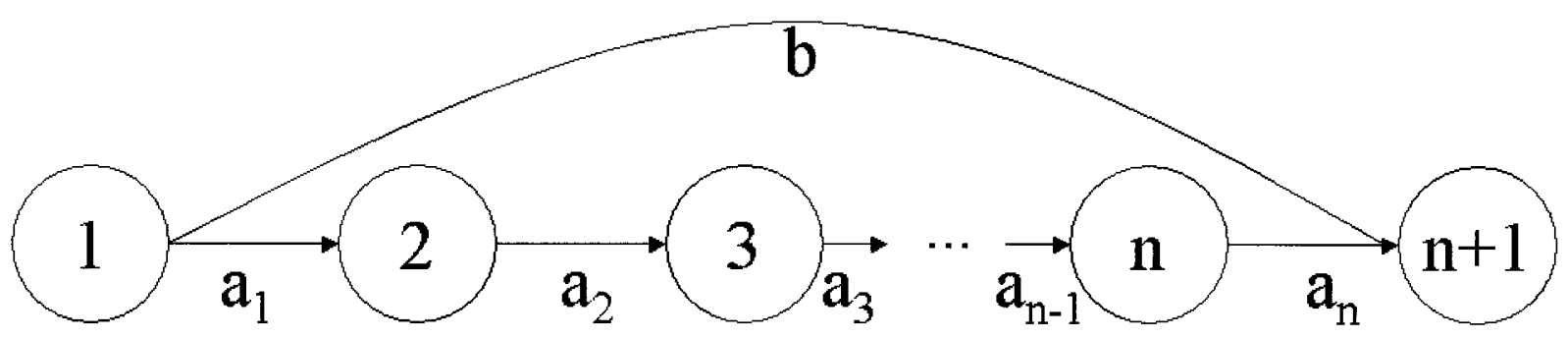

Figure 3.2: Nodes and connections in the $n$-hop communication.

For all the RSs in the system, the amount of data received and the amount of data transmitted must be equal:

$$
\operatorname{Data}(I N)=\operatorname{Data}(O U T)
$$

Total amount of data (in terms of bits), received or transmitted, can be expressed as follows:

$$
\text { Data }=\text { SpectralEfficiency }(\text { bits } / \mathrm{Hz} / \mathrm{sec}) \times \text { Time }(\mathrm{sec}) \times \text { BandWidth }(\mathrm{Hz}) .
$$

Then, from (3.1) \& (3.2),

$$
\left(S E_{I N}\right)\left(T_{I N}\right)\left(B_{I N}\right)=\left(S E_{\text {OUT }}\right)\left(T_{\text {OUT }}\right)\left(B_{\text {OUT }}\right)
$$

where $S E$ stands for spectral efficiency, $T$ for time, and $B$ for bandwidth. 
Assuming same amount bandwidth is used for both receiving and transmitting links,

$$
\begin{aligned}
& \left(S E_{I N}\right)\left(T_{I N}\right)=\left(S E_{\text {OUT }}\right)\left(T_{\text {OUT }}\right) \\
& \Rightarrow a_{1} \times T_{1}=a_{2} \times T_{2}=a_{3} \times T_{3}=\ldots=a_{n} \times T_{n}=b \times T .
\end{aligned}
$$

At this point it is clear that the total transmission time for single-hop communication is $T$, since there is only one link. However this is not the case for multi-hop communication. Each multi-hop link uses some amount of time for the transmission, i.e. ' $i$ 'th link uses $T_{i}$, and total transmission time for multi-hop communication is the 'union' of all time 'set's used for the link:

Total transmission time for multi-hop communication: $T_{1} \cup T_{2} \cup T_{3} \cup \ldots \cup T_{n}$ Total transmission time for single-hop communication: $T$.

It is aimed that the RSs would be simple and small devices compared to Base Stations, so they would need two separate channels. In this part, it is assumed a RS does not transmit and receive at the same time. Therefore, time 'set's of two adjacent links are orthogonal to each other. Moreover, in this part, it is also assumed that all other time 'set's for the same multi-hop communication are also considered as orthogonal to reduce the interference in the cell. Then,

Total transmission time for multi-hop communication: $T_{1}+T_{2}+T_{3}+\ldots+T_{n}$ Total transmission time for single-hop communication: $T$. 


\subsection{Comparison of the Data Rates}

Data rate can be thought as the amount of data (information) that can be transferred per unit time. Therefore to find the data rate for either communication types, i.e. multihop or single-hop, the total amount of data must be divided by the total amount of time. So, the ratio of multi-hop data rate to single-hop data can be found as follows:

$$
\frac{\text { Data Rate }_{\mathrm{MH}}}{\text { Data Rate }_{\mathrm{SH}}}=\frac{\text { Data }_{M H} / T_{M H}}{\operatorname{Data}_{S H} / T_{S H}} \text {, }
$$

where

$\operatorname{Data}_{M H}$ : Total amount of data for the Multi-hop transmission.

$T_{M H}:$ Total amount of time for the Multi-hop transmission.

$\operatorname{Data}_{S H}:$ Total amount of data for the Single-hop transmission.

$T_{S H} \quad$ : Total amount of time for the Single-hop transmission.

\subsection{1. $\quad$ Method 1}

For the same MS, to find the ratio of the data rates for multi-hop and single-hop cases in a fair manner, either total amount of data or total amount of time for both transmissions should be taken as equal. In this part, total amount of data is assumed to be equal for both cases:

$$
\operatorname{Data}_{M H}=\text { Data }_{S H} \text {. }
$$


Therefore, from (3.7) \& (3.8),

$$
\frac{\text { Data Rate }_{\mathrm{MH}}}{\text { Data Rate }_{\mathrm{SH}}}=\frac{1 / T_{M H}}{1 / T_{S H}}=\frac{T_{S H}}{T_{M H}} .
$$

Then,

$$
\frac{\text { Data Rate }_{\mathrm{MH}}}{\text { Data Rate }_{\mathrm{SH}}}=\frac{T}{T_{1}+T_{2}+T_{3}+\ldots+T_{n}}=\frac{\frac{T}{T_{1}}}{\frac{T_{1}}{T_{1}}+\frac{T_{2}}{T_{1}}+\frac{T_{3}}{T_{1}}+\ldots+\frac{T_{n}}{T_{1}}}
$$

From (3.4),

$$
\begin{aligned}
\frac{\text { Data Rate }_{\mathrm{MH}}}{\text { Data Rate }_{\mathrm{SH}}}= & \frac{\frac{a_{1}}{b}}{\frac{a_{1}}{a_{1}}+\frac{a_{1}}{a_{2}}+\frac{a_{1}}{a_{3}}+\ldots+\frac{a_{1}}{a_{n}}}=\frac{\frac{1}{b}}{\frac{1}{a_{1}}+\frac{1}{a_{2}}+\frac{1}{a_{3}}+\ldots+\frac{1}{a_{n}}} \\
= & \frac{\frac{1}{a_{1}}+\frac{1}{a_{2}}+\frac{1}{a_{3}}+\ldots+\frac{1}{a_{n}}}{b} .
\end{aligned}
$$

\subsection{2. $\quad$ Method 2}

Instead of assuming same amount of data transferred for single-hop and multi-hop cases, total transmission time can be taken as equal for both cases and same result can be obtained. Then for $T=T_{1}+T_{2}+T_{3}+\ldots+T_{n}$,

$$
\frac{\text { Data Rate }_{\mathrm{MH}}}{\text { Data Rate }_{\mathrm{SH}}}=\frac{\text { Data }_{M H}}{\text { Data }_{S H}} \text {. }
$$


From (3.2), single-hop data rate can be written as:

$$
\operatorname{Data}_{S H}=b T B_{S H}
$$

where

$b \quad:$ Spectral efficiency value for the single-hop communication.

T : Total amount of time required for single-hop communication.

$B_{S H} \quad:$ Bandwidth used for single-hop communication.

Since data from CN to MS, or vice versa, is being hopped through the RSs, total amount of data is equal to the total amount of data transmitted or received by any node (RS) on the route from $\mathrm{CN}$ to the MS. Then,

$$
\operatorname{Data}_{M H}=a_{i} T_{i} B_{M H} \quad i=1,2, \ldots, \mathrm{n},
$$

where

$a_{i} \quad:$ Spectral efficiency value for the ' $i$ ' th link of multi-hop communication.

$T_{i} \quad$ : Amount of time required for the transmission on ' $i$ ' th link.

$B_{M H} \quad$ : Bandwidth used for ' $i$ 'th link of multi-hop communication.

Then, assuming same amount of bandwidth is given for multi-hop and single-hop communication, the data rate ratio of multi-hop communication to that of single-hop communication becomes: 
$\frac{\text { Data Rate }_{\mathrm{MH}}}{\text { Data Rate }_{\mathrm{SH}}}=\frac{\text { Data }_{M H}}{\text { Data }_{S H}}=\frac{a_{i} T_{i}}{b T}=\frac{a_{i} T_{i}}{b \times\left(T_{1}+T_{2}+\ldots+T_{n}\right)}$.

The amount of data for all nodes is equal, so, from (3.4), instead of $a_{i} * T_{i}, a_{1} * T_{1}$ can be used. Then,

$$
\begin{aligned}
\frac{\text { Data Rate }_{\mathrm{MH}}}{\text { Data Rate }_{\mathrm{SH}}}= & \frac{a_{1} T_{1}}{b \times\left(T_{1}+T_{2}+\ldots+T_{n}\right)}=\frac{\frac{a_{1}}{b}}{\frac{T_{1}}{T_{1}}+\frac{T_{2}}{T_{1}}+\frac{T_{3}}{T_{1}}+\ldots+\frac{T_{n}}{T_{1}}} \\
= & \frac{\frac{a_{1}}{b}}{\frac{a_{1}}{a_{1}}+\frac{a_{1}}{a_{2}}+\frac{a_{1}}{a_{3}}+\ldots+\frac{a_{1}}{a_{n}}}=\frac{\frac{1}{b}}{\frac{1}{a_{1}}+\frac{1}{a_{2}}+\frac{1}{a_{3}}+\ldots+\frac{1}{a_{n}}} \\
= & \frac{\frac{1}{a_{1}}+\frac{1}{a_{2}}+\frac{1}{a_{3}}+\ldots+\frac{1}{a_{n}}}{b} .
\end{aligned}
$$

From (3.11) \& (3.16), it is seen that the same result can be obtained to find the ratio of multi-hop and singe-hop data rates by using either method:

$$
\frac{\text { Data Rate }_{\mathrm{MH}}}{\text { Data Rate }_{\mathrm{SH}}}=\frac{\frac{1}{\frac{1}{a_{1}}+\frac{1}{a_{2}}+\frac{1}{a_{3}}+\ldots+\frac{1}{a_{n}}}}{b} .
$$

In (3.17), single-hop data rate seems like proportional to the single-hop spectral efficiency, namely $b$, and multi-hop data rate is proportional to the result of some arithmetical operations of the composite multi-hop link spectral efficiency values. 
Therefore, to make it similar to the single-hop case, the result can be thought as 'combined spectral efficiency':

$$
\frac{\text { Data Rate }_{\mathrm{MH}}}{\text { Data Rate }_{\mathrm{SH}}}=\frac{a_{c}}{b}
$$

where $a_{c}$ is defined as combined ( $n$-hop) spectral efficiency and $a_{c}=\frac{1}{\sum_{i=1}^{n} \frac{1}{a_{i}}}$.

For multi-hop communication to be superior over single-hop communication, the multi-hop data rate must be higher than single-hop data rate. Therefore, the following constraint must be satisfied

$$
\frac{\text { Data Rate }_{\mathrm{MH}}}{\text { Data Rate }_{\mathrm{SH}}} \geq 1 \Rightarrow b \leq a_{c} \text {. }
$$

In order to deploy RSs efficiently, the closest distance from $\mathrm{CN}$ where multi-hop is superior over SH (in terms of data rate) should be found. At this point, it should be noticed that if all the links have same spectral efficiency value, then the best performance for multi-hop is achieved by 2-hop communication since the combined spectral efficiency is maximum for the smallest value of number of hops, namely $n$, for such a case. Therefore, the closest points to the $\mathrm{CN}$ where multi-hop communication is superior to single-hop communication must be through 2-hop communication where both CN-RS and RS-MS links has the maximum available spectral efficiency, i.e. $a_{i}=a_{\max }$. 


\subsection{Selection of Number of 'Hop's}

\subsubsection{2-Hop Case}

For 2-hop communication (3.19) becomes $b \leq a_{\max } / 2$. That is, for the usage of 2-hop communication the threshold single-hop spectral efficiency value is $a_{\max } / 2$. In other words, if the spectral efficiency for single-hop communication is greater than or equal to $a_{\max } / 2$, multi-hop communication, even for the best case (2-hop communication where both links have spectral efficiency of $a_{\max }$ which results in a combined spectral efficiency of $a_{\max } / 2$ ), cannot improve the data rate of the transmission; so it's better to use singlehop communication for MSs having single-hop spectral efficiency greater than $a_{\max } / 2$. On the other hand, if the spectral efficiency for single-hop communication is less than $a_{\max } / 2$, best case of 2-hop communication becomes superior over single-hop communication, enabling the use of 2-hop communication in that region if combined spectral efficiency of multi-hop links, i.e. CN-RS \& RS-MS links, is greater than the spectral efficiency of the single-hop link, i.e. CN-MS link, for the same MS.

Figure 3.3 shows an example of 2-hop communication system. If the spectral efficiency of the $\mathrm{CN}-\mathrm{MS}$ link, namely $b$ in the figure, is greater than $a_{\max } / 2$, there is no need to look for any kind of multi-hop communication since the performance of single-hop communication cannot be outperformed in such case. However, if $b$ is less than $a_{\max } / 2$, there is a chance that multi-hop (in this case 2-hop) communication may perform better, so, for that MS, combined spectral efficiency has to be calculated and 
compared with $b$ to decide whether single-hop or 2-hop is to be used. The combined spectral efficiency for 2-hop case is:

$$
a_{c}=\frac{1}{\frac{1}{a_{1}}+\frac{1}{a_{2}}} \text {. }
$$

Then, in short, the selection criterion becomes:

$$
\begin{array}{lll}
a_{c} \leq b & \Rightarrow & \text { Single-hop } \\
a_{c}>b & \Rightarrow & \text { 2-hop. }
\end{array}
$$

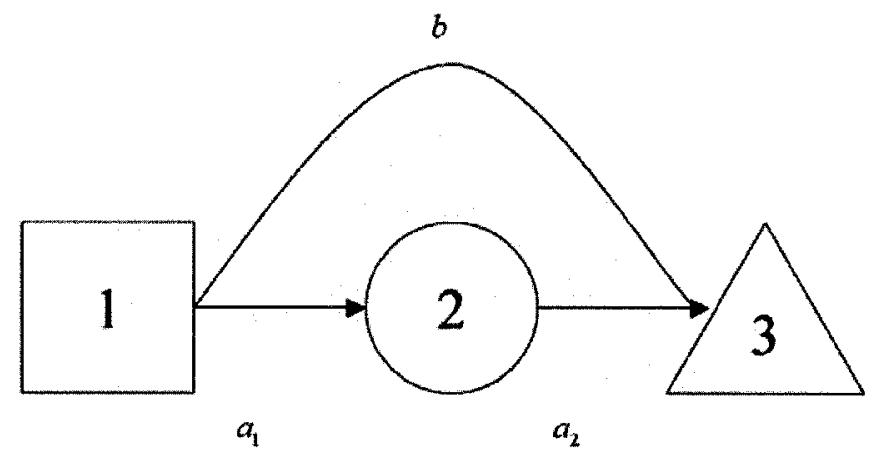

Figure 3.3: Nodes and links for 2-hop case.

\subsubsection{3-Hop Case}

For 3-hop communication (3.19) becomes $b \leq a_{\max } / 3$. That is, for the usage of 3-hop communication the threshold single-hop spectral efficiency value is $a_{\max } / 3$. Similar to 2 hop case, if the spectral efficiency for single-hop communication is greater than or equal to $a_{\max } / 3,3-$ hop communication, even for the best case (where all links have spectral efficiency of $a_{\max }$ which results a combined spectral efficiency of $a_{\max } / 3$ ), cannot 
improve the data rate of the transmission; so it's better to use single-hop communication (or if applicable 2-hop communication in case 2-hop communication has higher spectral efficiency than the single-hop case). On the other hand, if the spectral efficiency for single-hop communication is less than $a_{\max } / 3$, best case of 3-hop communication becomes superior over single-hop communication, enabling the use of 3-hop communication in that region if combined spectral efficiency composite multi-hop links is greater than the CN-MS link single-hop spectral efficiency for the same MS. Of course, at that point, the combined spectral efficiency of a 2-hop communication for the same MS should be checked, and if that value is greater than the combined spectral efficiency of 3-hop communication, 2-hop communication should be used for that MS. Figure 3.4 shows an example of 3-hop communication system. In the figure:

$b \quad$ : Single-hop spectral efficiency between node 1 and node 4 .

$a_{13} \quad$ : Direct link spectral efficiency between node 1 and node 3 .

$a_{24} \quad$ : Direct link spectral efficiency between node 2 and node 4 .

Following definitions are made for Figure 3.4 as well:

$$
a_{134}=\frac{1}{\frac{1}{a_{13}}+\frac{1}{a_{3}}} \quad \& \quad a_{124}=\frac{1}{\frac{1}{a_{1}}+\frac{1}{a_{24}}}
$$

where $a_{134}$ is the combined spectral efficiency of the composite CN-RS $-\mathrm{MS}$ link and $a_{124}$ is the combined spectral efficiency of the composite CN-RS - MS link. 


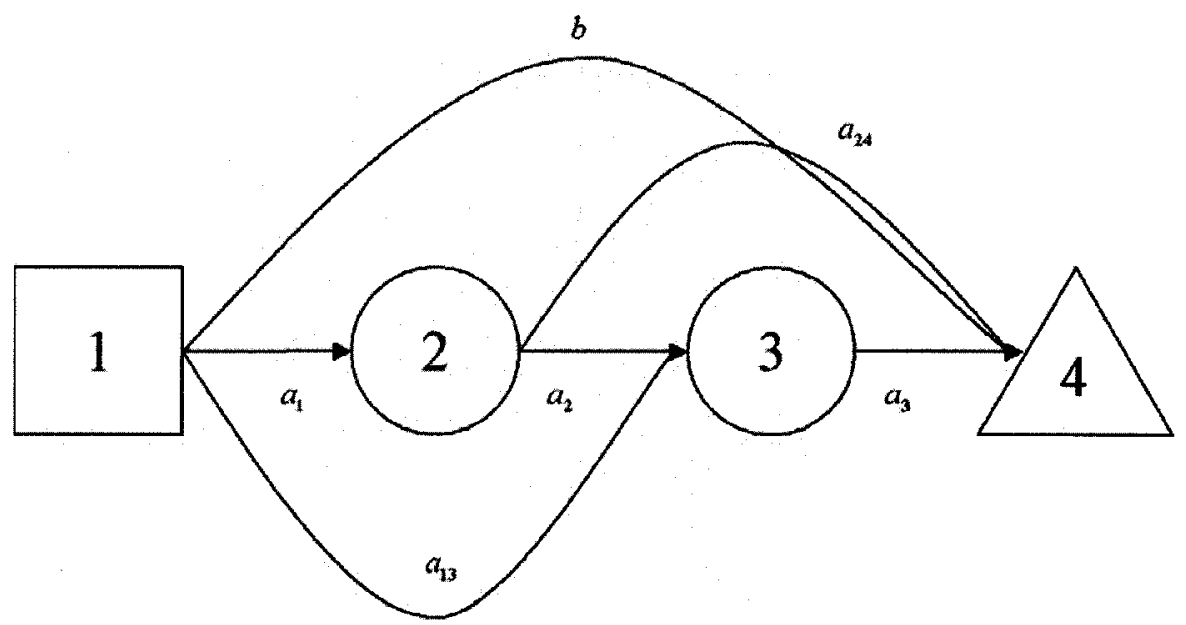

Figure 3.4: Nodes and links for 3-hop case.

As seen from Figure 3.3, there are four possible routes for CN-MS communication.

Table 3.1 shows the possible links for 3-hop case.

Table 3.1: Possible Links for CN-MS Communication.

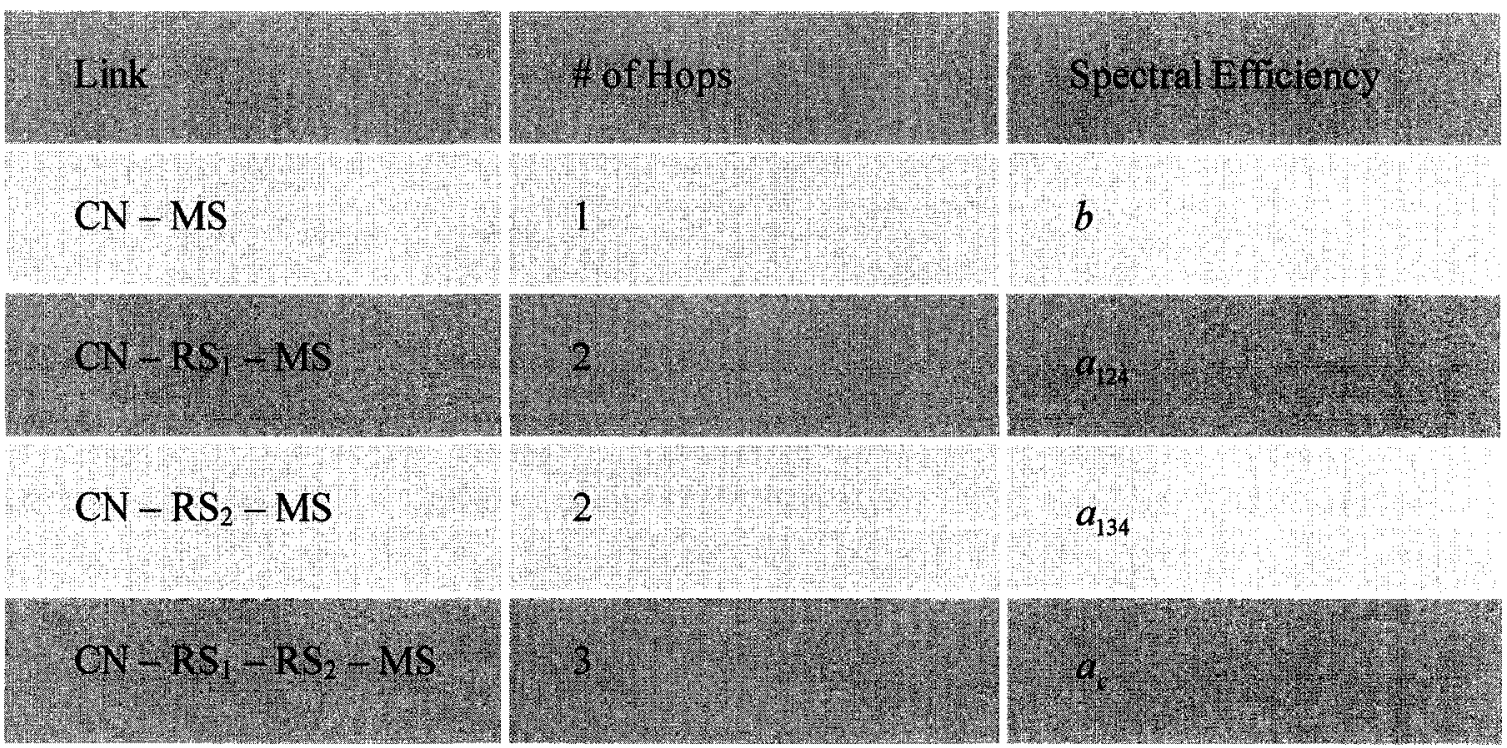


In order to obtain the best performance for that MS, all four possible routes should be considered. There are three cases depending on the value of the spectral efficiency, $b$, of single-hop communication for that MS:

- If $b \geq a_{\max } / 2$, there is no need to consider any of the multi-hop routes since they cannot perform better than the single-hop one.

- If $\left(a_{\max } / 2\right)>b \geq\left(a_{\max } / 3\right)$, there is a possibility that combined spectral efficiency values of 2-hop links, $a_{124}$ and/or $a_{134}$, may be higher than the spectral efficiency of the single-hop link, so those three values should be compared to find the best link. However, it should be noted that since the combined spectral efficiency of 3 -hop link cannot exceed $a_{\max } / 3$, there is no need to take 3-hop cases into consideration.

- If $b<a_{\max } / 3$, any of the four routes has the possibility to be the best one in terms of spectral efficiency, thus all three combined spectral efficiencies and the single-hop spectral efficiency should be compared to find the one that perform best.

\subsection{3. n-Hop Case}

For $n$-hop communication (3.19) becomes $b \leq a_{\max } / n$. That is, for the usage of $n$-hop communication the threshold single-hop spectral efficiency value is $a_{\max } / n$; if the spectral efficiency for single-hop communication is greater than or equal to $a_{\max } / n, n$ - 
hop communication, even for the best case (where all links have spectral efficiency of $a_{\max }$ which results a combined spectral efficiency of $\left.a_{\max } / n\right)$, cannot improve the data rate of the transmission; so it's better to use single-hop communication or if applicable one of the 2-hop/3-hop/.../(n-I)-hop communication options.

\subsection{Finding the Radii of Spectral Efficiency Circles}

The first step of the optimization process of RS position is to find the closest distance for relay assistance and then, in general, to find the regions where ' $i$ '-hop is applicable in the cell. These regions are directly related to the spectral efficiency of the single-hop link between the $\mathrm{CN}$ and the MS. Figure 3.5 shows an (non-scaled) illustration of spectral efficiency coverage circles for maximum spectral efficiency value of $6 \mathrm{bits} / \mathrm{sec} / \mathrm{Hz}$.

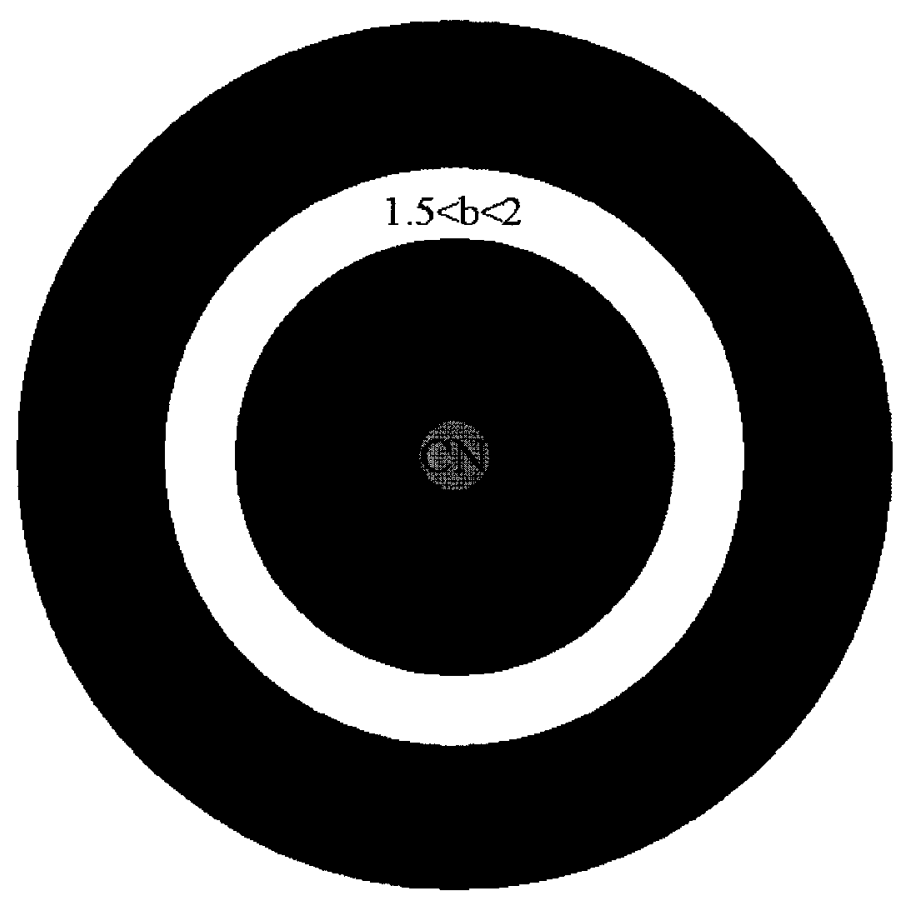

Figure 3.5: Spectral efficiency circles for $a_{\max }=6 \mathrm{bits} / \mathrm{sec} / \mathrm{Hz}$. 
- In Figure 3.5, red region, i.e. area of the inner most circle, represents the region where single-hop spectral efficiency is greater than $3 \mathrm{bits} / \mathrm{sec} / \mathrm{Hz}$; in that region none of the multi-hop cases can perform better than the single-hop case, so, MSs in that region should use the direct link with the $\mathrm{CN}$ (single-hop communication).

- In the orange region, the spectral efficiency of single-hop communication is between 2 and 3, so in that region 3-hop communication cannot perform better than single-hop communication, then MSs in that region should use single-hop or 2-hop depending on their combined spectral efficiency values.

- In the yellow region, the spectral efficiency of single-hop communication is between 1.5 and 2 , so in that region 4-hop communication cannot perform better than single-hop communication, then MSs in that region should use single-hop, 2-hop, or 3-hop depending on their combined spectral efficiency values.

- In the green region, the spectral efficiency of single-hop communication is between 1.2 and 1.5 , so in that region 5-hop communication cannot perform better than single-hop communication, then MSs in that region should use single-hop, 2-hop, 3-hop or 4-hop depending on their combined spectral efficiency values, and so on. 
Let's assume the radius of the circle around the red region is $r_{1}$, the radius of the circle around the orange region is $r_{2}$, and so on. The radii of those circles are determined by the values of spectral efficiencies. It is known that there is a relation between spectral efficiency and SINR; Table 3.2 and Figure 3.6 show this relation.

Table 3.2: Relation of all combinations, required SINR and spectral efficiency that will yield $B E R$ of $10^{-5}$.

\begin{tabular}{ccc}
\hline Combinations of modulation and & $\begin{array}{c}\text { Minimum Required } \\
\text { code rates }\end{array}$ & $\begin{array}{c}\text { Spectral Efficiency } \\
\text { [bits/sec/Hz] }\end{array}$ \\
\hline QPSK, rate: $1 / 2$ & 4 & 1 \\
QPSK, rate: $2 / 3$ & 6 & 1.33 \\
QPSK, rate: $3 / 4$ & 6.8 & 1.5 \\
QPSK, rate: $7 / 8$ & 7.8 & 1.75 \\
16-QAM, rate: $1 / 2$ & 10 & 2 \\
16-QAM, rate: $2 / 3$ & 12 & 2.67 \\
16-QAM, rate: $3 / 4$ & 13 & 3 \\
16-QAM, rate: $7 / 8$ & 15 & 3.5 \\
64-QAM, rate: $2 / 3$ & 17.7 & 4 \\
64-QAM, rate: $3 / 4$ & 19.4 & 4.5 \\
64-QAM, rate: $7 / 8$ & 21 & 5.25 \\
64-QAM, rate: 1 & 26 & 6 \\
\hline
\end{tabular}




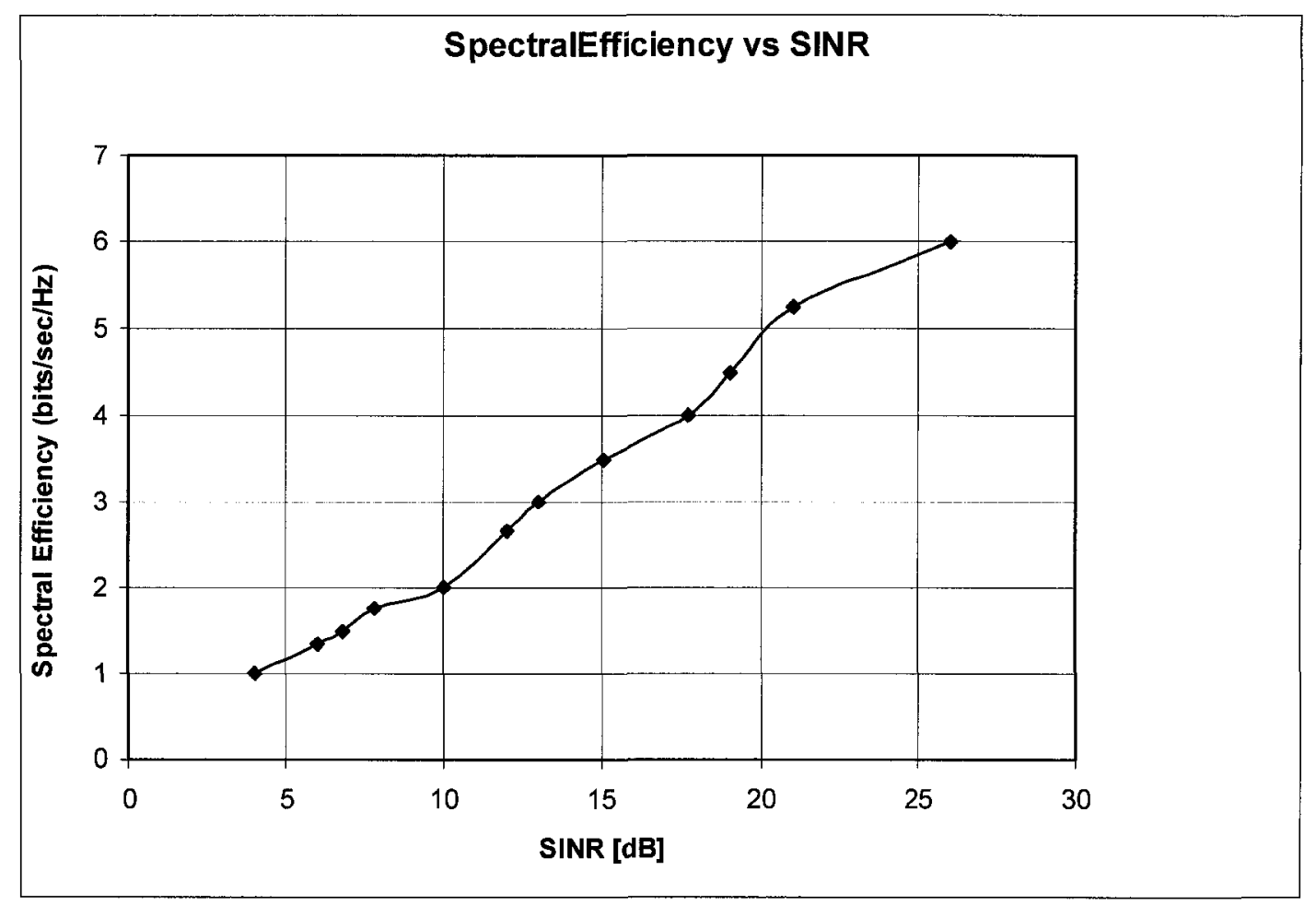

Figure 3.6: Spectral efficiency vs. SINR graph.

Signal-to-interference-plus-noise ratio (SINR) can be written as follows:

$$
\gamma^{d B}=10 \log \frac{P_{S}}{P_{I}+P_{N}} \Rightarrow P_{S}=\left(P_{I}+P_{N}\right) 10^{\frac{\gamma^{d B}}{10}}
$$

where $\gamma^{d B}$ is SINR in $\mathrm{dB}, P_{S}$ is the received signal power, $P_{I}$ is the interference power and $P_{N}$ is the noise power. The received signal power can be written as follows (shadowing effect is ignored):

$$
P_{S}=\alpha \frac{P_{C N}}{r^{n}}
$$


where $P_{C N}$ is the transmit power of the $\mathrm{CN}, r$ is the transmission distance, $\alpha$ is a constant, and $n$ is the propagation constant. Then,

$$
\begin{aligned}
& \alpha \frac{P_{C N}}{r^{n}}=\left(P_{I}+P_{N}\right) 10^{\frac{\gamma^{A B}}{10}} \\
& \Rightarrow r^{n}=\alpha \frac{P_{C N}}{\left(P_{I}+P_{N}\right) 10^{\frac{\gamma^{d B}}{10}}} .
\end{aligned}
$$

At this point, to simplify the analysis, some assumptions are made:

- Noise power in (3.24) is assumed to be uniform throughout the cell; in other words, all the MSs are faced with the same amount of noise power.

- Similarly, interference power in (3.24) is assumed to be uniform throughout the cell.

Therefore, (3.24) can be modified as follows:

$$
r^{n}=\alpha \frac{P_{C N}}{P_{N I} 10^{\frac{\gamma^{d B}}{10}}} .
$$

where $P_{N I}$ is the uniform noise-plus-interference power.

Equation (3.25) can be used to find the radii of the coverage circles. As mentioned before, $r_{1}$ corresponds to the radius of the region where spectral efficiency is greater than or equal to $a_{\max } / 2$. In Table $2, a_{\max }$ is $6 \mathrm{bits} / \mathrm{sec} / \mathrm{Hz}$, so $a_{\max } / 2$ corresponds to 3 bits/sec/Hz, which requires SINR value of $13 \mathrm{~dB}$ for $16-\mathrm{QAM}$ rate $(3 / 4)$, 


$$
r_{1}^{n}=\alpha \frac{P_{C N}}{P_{N I} 10^{\frac{13}{10}}}
$$

Similarly, $r_{2}$ corresponds to the region where spectral efficiency greater than 2 , which correspond to SINR value greater than $10 \mathrm{~dB}$ for 16 -QAM rate $(1 / 2)$,

$$
r_{2}^{n}=\alpha \frac{P_{C N}}{P_{N I} 10^{\frac{10}{10}}} .
$$

Then,

$$
\Rightarrow \frac{r_{2}^{n}}{r_{1}^{n}}=\frac{10^{\frac{13}{10}}}{10^{\frac{10}{10}}}=10^{\frac{3}{10}} \Rightarrow \frac{r_{2}}{r_{1}}=10^{\frac{3}{10 n}} .
$$

Let's assume that the coverage are of the cell is where spectral efficiency is greater than 1 or SINR greater than $4 \mathrm{~dB}$ (for QPSK rate $(1 / 2)$ ), i.e. the area inside the radius of the circle surrounding blue region. Then,

$$
\begin{gathered}
r_{\text {cell }}^{n}=\alpha \frac{P_{C N}}{P_{N I} 10^{\frac{4}{10}}} \\
\Rightarrow \frac{r_{\text {cell }}}{r_{1}}=10^{\frac{9}{10 n}} .
\end{gathered}
$$


For urban areas it is reasonable to assume propagation constant equal to 4 :

$$
\begin{array}{lll}
\frac{r_{2}}{r_{1}}=10^{\frac{3}{10 \times 4}}=1.1885 & ; & \frac{r_{3}}{r_{1}}=10^{\frac{6.2}{10 \times 4}}=1.4289 \\
\frac{r_{4}}{r_{1}}=10^{\frac{7.8}{10 \times 4}}=1.5667 & ; & \frac{r_{\text {cell }}}{r_{1}}=10^{\frac{9}{10 \times 4}}=1.6788 .
\end{array}
$$

Table 3.3: Spectral Efficiency Circles Radii Ratios.

\begin{tabular}{|l|l|l|l|l|l|}
\hline $\mathbf{n}$ & $\mathbf{4 . 5}$ & $\mathbf{4}$ & $\mathbf{3 . 5}$ & $\mathbf{3}$ & $\mathbf{2 . 5}$ \\
\hline $\mathbf{r} 2 / \mathbf{r 1}$ & 1.1659 & 1.1885 & 1.2182 & 1.2589 & 1.3182 \\
\hline $\mathbf{r 3} / \mathbf{r 1}$ & 1.3733 & 1.4289 & 1.5036 & 1.6094 & 1.7701 \\
\hline $\mathbf{r 4} / \mathbf{r 1}$ & 1.4905 & 1.5667 & 1.6705 & 1.8197 & 2.0511 \\
\hline $\mathbf{r c e l 1} / \mathbf{r 1}$ & 1.5849 & 1.6788 & 1.8077 & 1.9952 & 2.2908 \\
\hline
\end{tabular}

Figure 3.7 shows the spectral efficiency circle radii for propagation constant value of 3.5. The single-hop spectral efficiency in the area inside the innermost circle is greater than 3 ; in the area between the inner most and the second circle the value is between 2 and 3 , and so on. It should be noted that this figure is Figure 3.5 drawn to scale. 


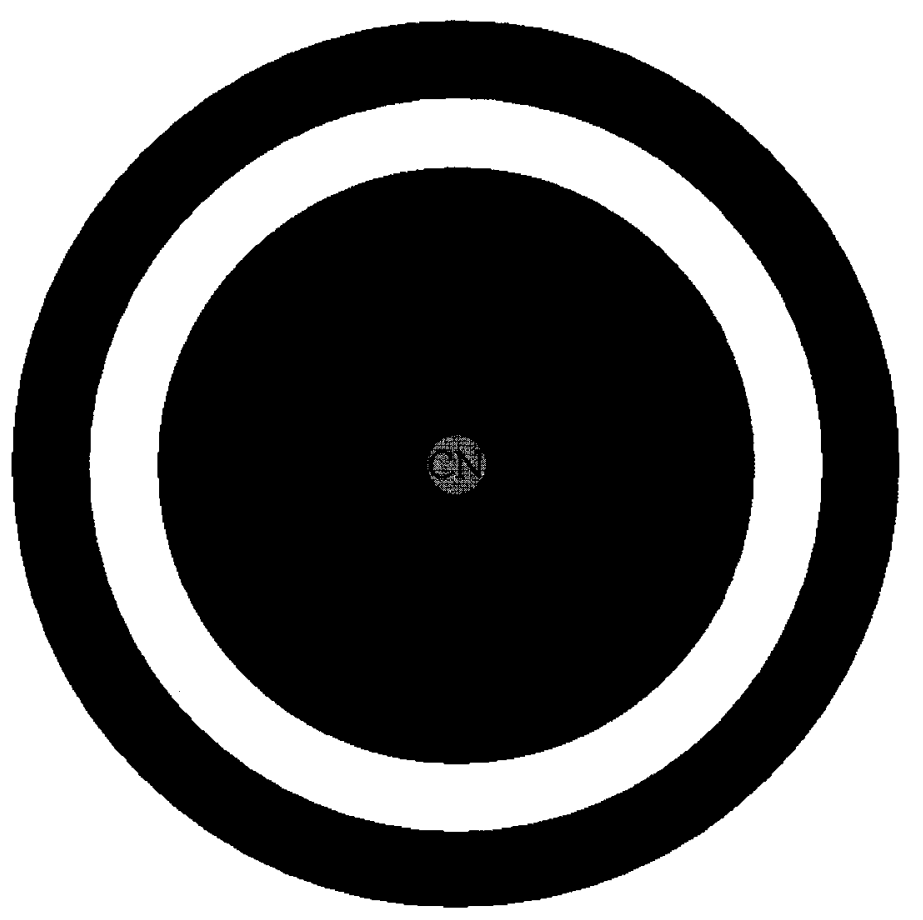

Figure 3.7: Spectral efficiency coverage circles for $n=3.5$.

\subsection{Optimization of the RS Positions}

Cell area can be divided into two regions, $R l$ and $R 2$, as shown in Figure 3.8 . The radius of the inner circle is $r$ and the radius for the outer one is $R$. The MSs in $R 1$ get a spectral efficiency greater than or equal to $a_{\max } / 2$ from the $\mathrm{CN}$. Therefore, for those MSs single-hop communication is superior to any kind of multi-hop communication. On the other hand, in $R 2$, depending on the combined spectral efficiency, multi-hop communication may be superior to single-hop communication. The ultimate aim, in this case, is to provide the maximum attainable spectral efficiency to the MSs in $R 2$, e.g. 
$a_{\max } / 2$ for 2-hop communication, via multi-hop; MSs in $R 1$ already get the maximum attainable spectral efficiency via single-hop communication.

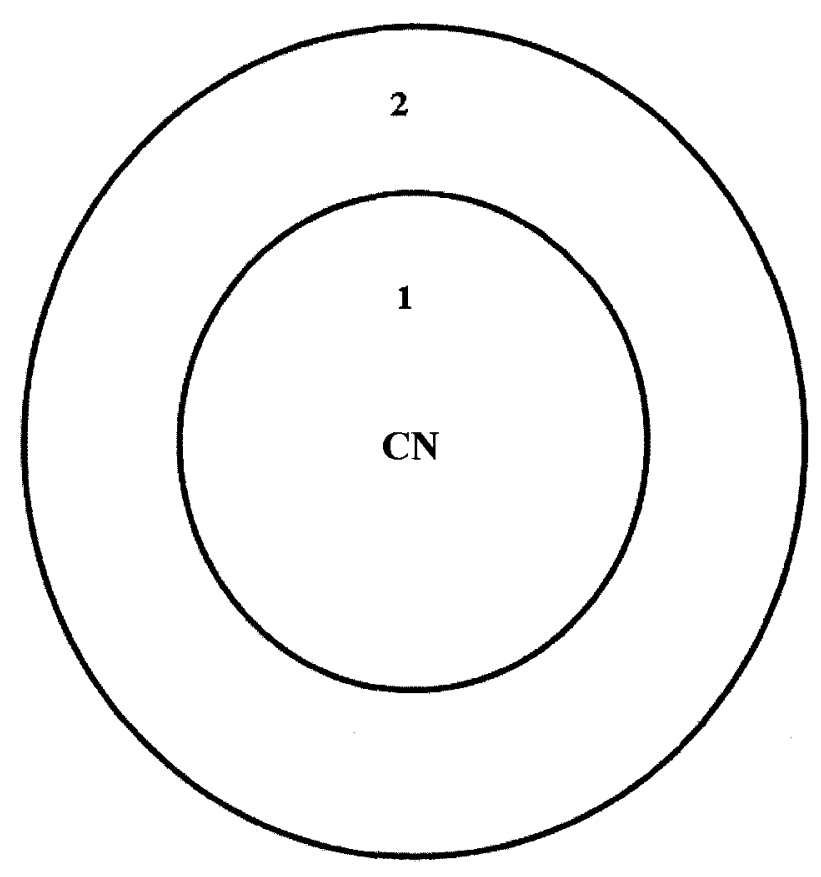

Figure 3.8: Region 1 and Region 2 for $n=4$.

To start with, consider a MS in $R 2$ but just on the border of $R 1$. This MS can get a spectral efficiency of almost $a_{\max } / 2$ from the CN using single-hop. If multi-hop is to be used for the communication of this MS, same spectral efficiency should be provided. So, if the nearest RS is located at a distance of $(r+d)$ from the $\mathrm{CN}$, that RS should provide spectral efficiency of $a_{\max }$ at the distance $d$, just on the border of $R 1$. Therefore, that MS would be provided with the maximum combined spectral efficiency for multi-hop communications, which is $a_{\max } / 2$. 
Similar to the, previously done, spectral efficiency circle radius finding calculations, $d$ can be found as follows:

$$
\begin{gathered}
d^{n}=\frac{P_{R S}}{\left(P_{I}+P_{N}\right) 10^{\frac{\gamma_{1}^{d B}}{10}}} \\
r_{1}^{n}=\frac{P_{C N}}{\left(P_{I}+P_{N}\right) 10^{\frac{\gamma_{2}^{d B}}{10}}},
\end{gathered}
$$

where $\gamma_{1}^{d B}$ is the SINR value corresponding to the maximum spectral efficiency, $a_{\max }$, and $\gamma_{2}^{d B}$ is the SINR value corresponding to half of the maximum spectral efficiency, $a_{\max } / 2$. From the two equations,

$$
\left(\frac{d}{r_{1}}\right)^{n}=\frac{P_{R S}}{P_{C N}} 10^{\frac{\gamma_{2}^{d B}-\gamma_{1}^{d B}}{10}} .
$$

For $a_{\max }=6, \gamma_{1}^{d B}$ and $\gamma_{2}^{d B}$ values are $26 \mathrm{~dB}$ and $13 \mathrm{~dB}$, respectively. Then, (3.32) becomes:

$$
\left(\frac{d}{r_{1}}\right)^{n}=\frac{P_{R S}}{P_{C N}} 10^{-1.3} .
$$

For different values of the propagation constant $n$ and the ratio $P_{R S} / P_{C N}$, the radius $d$ can be found in terms of $r_{1}$. Table 3.4 shows the number and position of the first tier RSs for various system parameters. Following definitions are used in the table: 
r1: Ratio of RS coverage radius to inner circle radius.

$(\mathbf{2 d}+\mathbf{r 1}) / \mathbf{r 1}$ : Maximum distance from $\mathrm{CN}$ that the MSs are provided with spectral efficiency greater than (for single-hop) or equal (for 2-hop) to 3.

$\mathbf{R} / \mathbf{r 1}$ : Ratio of cell radius to innermost circle radius.

SINR by the RS at the cell border: The SINR value for the link between RS and a MS positioned at the cell border.

SE by the RS at the cell border: Spectral Efficiency of link between RS and a MS positioned at the cell border.

CSE of the RS at cell border: Combined Spectral Efficiency of 2-hop communication for a MS positioned at the cell border.

Table 3.4: Position and number of the first tier RSs.

\begin{tabular}{|c|c|c|c|c|c|}
\hline $\mathbf{n}$ & 4.5 & 4 & 3.5 & 3 & 2.5 \\
\hline r2/r1 & 1.16 & 1.18 & 1.21 & 1.25 & 1.31 \\
\hline $\mathbf{r 3 / r 1}$ & 1.37 & 1.42 & 1.50 & 1.60 & 1.77 \\
\hline r4/r1 & 1.49 & 1.56 & 1.67 & 1.81 & 2.05 \\
\hline $\mathrm{d} / \mathrm{r} 1$ for $\operatorname{Prs} / \operatorname{Pcn}=0.05$ & 0.26 & 0.22 & 0.18 & 0.13 & 0.09 \\
\hline$(2 \mathrm{~d}+\mathrm{r} 1) / \mathrm{r} 1$ for $\operatorname{Prs} / \operatorname{Pcn}=0.05$ & 1.52 & 1.44 & 1.36 & 1.27 & 1.18 \\
\hline rcell//r1 & 1.58 & 1.67 & 1.80 & 1.99 & 2.29 \\
\hline SINR by the RS at cell border & 22.21 & 13.66 & 7.08 & 1.96 & -1.98 \\
\hline SE of the RS at the cell border & 5.4 & 3.2 & 1.6 & - & - \\
\hline CSE of RS at cell border & 2.84 & 2.09 & 1.26 & - & - \\
\hline Number of RSs needed & 17.3 & 19.8 & 23.7 & 30.31 & 43.4 \\
\hline $\mathrm{d} / \mathrm{r} 1$ for $\operatorname{Prs} / \operatorname{Pcn}=0.1$ & 0.30 & 0.26 & 0.22 & 0.17 & 0.12 \\
\hline$(2 d+r 1) / r 1$ for $\operatorname{Prs} / \operatorname{Pcn}=0.1$ & 1.61 & 1.53 & 1.44 & 1.34 & 1.24 \\
\hline rcell/r1 & 1.58 & 1.67 & 1.80 & 1.99 & 2.29 \\
\hline SINR by the RS at cell border & 28.10 & 18.37 & 11.08 & 5.52 & 1.28 \\
\hline SE of the RS at the cell border & - & 4.2 & 2.3 & 1.2 & - \\
\hline CSE of RS at cell border & - & 2.47 & 1.66 & 1 & - \\
\hline
\end{tabular}




\begin{tabular}{|c|c|c|c|c|c|}
\hline Number of RSs needed & 15.4 & 17.2 & 20.1 & 24.8 & 33.8 \\
\hline $\mathrm{d} / \mathrm{r} 1$ for $\operatorname{Prs} / \operatorname{Pcn}=0.2$ & 0.35 & 0.31 & 0.26 & 0.21 & 0.15 \\
\hline$(2 \mathrm{~d}+\mathrm{r} 1) / \mathrm{r} 1$ for $\operatorname{Prs} / \operatorname{Pcn}=0.2$ & 1.71 & 1.63 & 1.53 & 1.43 & 1.31 \\
\hline rcell $/ \mathbf{r} 1$ & 1.58 & 1.67 & 1.80 & 1.99 & 2.29 \\
\hline SINR by the RS at cell border & - & 23.64 & 15.39 & 9.25 & 4.66 \\
\hline SE of the RS at the cell border & - & 5.75 & 3.6 & 1.9 & 1.1 \\
\hline CSE of RS at cell border & - & 2.94 & 2.25 & 1.44 & 0.93 \\
\hline Number of RSs needed & 13.7 & 15.1 & 17.1 & 20.4 & 26.5 \\
\hline $\mathrm{d} / \mathrm{rl}$ for $\operatorname{Prs} / \operatorname{Pcn}=0.3$ & 0.39 & 0.35 & 0.30 & 0.24 & 0.18 \\
\hline$(2 \mathrm{~d}+\mathrm{r} 1) / \mathrm{r} 1$ for $\operatorname{Prs} / \operatorname{Pcn}=0.3$ & 1.78 & 1.70 & 1.60 & 1.49 & 1.37 \\
\hline rcell/r1 & 1.58 & 1.67 & 1.80 & 1.99 & 2.29 \\
\hline SINR by the RS at cell border & - & - & 18.11 & 11.54 & 6.69 \\
\hline SE of the RS at the cell border & - & - & 4.1 & 2.5 & 1.5 \\
\hline CSE of RS at cell border & - & - & 2.44 & 1.76 & 1.2 \\
\hline Number of RSs needed & 12.8 & 13.9 & 15.6 & 18.3 & 23.0 \\
\hline $\mathrm{d} / \mathrm{r} 1$ for $\mathrm{Prs} / \mathrm{Pcn}=0.4$ & 0.41 & 0.37 & 0.32 & 0.27 & 0.20 \\
\hline$(2 d+r 1) / r 1$ for $\operatorname{Prs} / \operatorname{Pcn}=0.4$ & 1.83 & 1.75 & 1.65 & 1.54 & 1.41 \\
\hline rcell/r1 & 1.58 & 1.67 & 1.80 & 1.99 & 2.29 \\
\hline SINR by the RS at cell border & - & - & 20.16 & 13.23 & 8.16 \\
\hline SE of the RS at the cell border & - & - & 5 & 3.1 & 1.8 \\
\hline CSE of RS at cell border & - & - & 2.73 & 2 & 1.38 \\
\hline Number of RSs needed & 12.2 & 13.2 & 14.7 & 16.9 & 20.9 \\
\hline $\mathrm{d} / \mathrm{r} 1$ for $\operatorname{Prs} / \operatorname{Pcn}=0.5$ & 0.44 & 0.39 & 0.34 & 0.2926 & 0.22 \\
\hline$(2 \mathrm{~d}+\mathrm{r} 1) / \mathrm{r} 1$ for $\operatorname{Prs} / \operatorname{Pcn}=0.5$ & 1.88 & 1.79 & 1.69 & 1.5852 & 1.45 \\
\hline rcell/r1 & 1.58 & 1.67 & 1.80 & 1.9952 & 2.29 \\
\hline SINR by the RS at cell border & - & - & 21.82 & 14.5866 & 9.33 \\
\hline SE of the RS at the cell border & - & - & 5.3 & 3.4 & 1.9 \\
\hline CSE of RS at cell border & - & - & 2.81 & 2.17 & 1.44 \\
\hline Number of RSs needed & 11.8 & 12.7 & 14.0 & 16.0 & 19.5 \\
\hline
\end{tabular}




\begin{tabular}{|c|c|c|c|c|c|}
\hline $\mathrm{d} / \mathrm{r} 1$ for $\operatorname{Prs} / \operatorname{Pcn}=0.6$ & 0.45 & 0.41 & 0.36 & 0.31 & 0.24 \\
\hline$(2 d+r 1) / r 1$ for $\operatorname{Prs} / \mathbf{P c n}=0.6$ & 1.91 & 1.83 & 1.73 & 1.62 & 1.49 \\
\hline $\mathbf{r c e l l} / \mathbf{r} 1$ & 1.58 & 1.67 & 1.80 & 1.99 & 2.29 \\
\hline SINR by the RS at cell border & - & - & 23.25 & 15.72 & 10.30 \\
\hline SE of the RS at the cell border & - & - & 5.7 & 3.6 & 2.1 \\
\hline CSE of RS at cell border & - & - & 2.92 & 2.25 & 1.55 \\
\hline Number of RSs needed & 11.5 & 12.3 & 13.5 & 15.3 & 18.3 \\
\hline $\mathrm{d} / \mathrm{r} 1$ for $\operatorname{Prs} / \operatorname{Pcn}=0.7$ & 0.47 & 0.43 & 0.38 & 0.32 & 0.26 \\
\hline$(2 d+r 1) / r 1$ for $\operatorname{Prs} / \mathbf{P c n}=0.7$ & 1.95 & 1.86 & 1.76 & 1.65 & 1.52 \\
\hline rcell/r1 & 1.58 & 1.67 & 1.80 & 1.99 & 2.29 \\
\hline SINR by the RS at cell border & - & - & 24.50 & 16.61 & 11.14 \\
\hline SE of the RS at the cell border & - & - & 5.8 & 3.8 & 2.35 \\
\hline CSE of RS at cell border & - & - & 2.95 & 2.33 & 1.7 \\
\hline Number of RSs needed & 11.2 & 11.9 & 13.1 & 14.7 & 17.5 \\
\hline $\mathrm{d} / \mathrm{r} 1$ for $\operatorname{Prs} / \operatorname{Pcn}=0.8$ & 0.48 & 0.44 & 0.39 & 0.34 & 0.27 \\
\hline$(2 \mathrm{~d}+\mathrm{r} 1) / \mathrm{r} 1$ for $\operatorname{Prs} / \operatorname{Pcn}=0.8$ & 1.97 & 1.89 & 1.79 & 1.68 & 1.55 \\
\hline rcell/r1 & 1.58 & 1.67 & 1.80 & 1.99 & 2.29 \\
\hline SINR by the RS at cell border & - & - & 25.62 & 17.58 & 11.87 \\
\hline SE of the RS at the cell border & - & - & 5.9 & 3.9 & 2.6 \\
\hline CSE of RS at cell border & - & - & 2.97 & 2.36 & 1.81 \\
\hline Number of RSs needed & 11.0 & 11.7 & 12.7 & 14.2 & 16.7 \\
\hline $\mathrm{d} / \mathrm{r1}$ for $\operatorname{Prs} / \operatorname{Pcn}=0.9$ & 0.50 & 0.46 & 0.41 & 0.35 & 0.28 \\
\hline$(2 \mathrm{~d}+\mathrm{r} 1) / \mathrm{r} 1$ for $\operatorname{Prs} / \operatorname{Pcn}=0.9$ & 2.00 & 1.92 & 1.82 & 1.71 & 1.57 \\
\hline rcell/r1 & 1.58 & 1.67 & 1.80 & 1.99 & 2.29 \\
\hline SINR by the RS at cell border & - & - & - & 18.37 & 12.52 \\
\hline SE of the RS at the cell border & - & - & - & 4.3 & 2.8 \\
\hline CSE of RS at cell border & - & - & - & 2.5 & 1.9 \\
\hline Number of RSs needed & 10.8 & 11.5 & 12.4 & 13.8 & 16.1 \\
\hline $\mathrm{d} / \mathrm{r} 1$ for $\operatorname{Prs} / \operatorname{Pen}=1$ & 0.51 & 0.47 & 0.42 & 0.36 & 0.30 \\
\hline$(2 d+r 1) / r 1$ for $\operatorname{Prs} / \operatorname{Pcn}=1$ & 2.02 & 1.94 & 1.85 & 1.73 & 1.60 \\
\hline
\end{tabular}




\begin{tabular}{|c|c|c|c|c|c|} 
rcell/r1 & 1.58 & 1.67 & 1.80 & 1.99 & 2.29 \\
\hline SINR by the RS at cell border & - & - & - & 19.09 & 13.12 \\
\hline SE of the RS at the cell border & - & - & - & 4.5 & 3 \\
\hline CSE of RS at cell border & - & - & - & 2.57 & 2 \\
\hline Number of RSs needed & 10.6 & 11.3 & 12.1 & 13.4 & 15.6 \\
\hline
\end{tabular}

Figures $3.9 \& 3.10$ show the cell dimensions and RS positions according to the values from Table 3.4 for propagation constant values of 4 and 3. As the reference dimension, the value of $r_{1}$, radius of the circle in which the CN serves spectral efficiency of $a_{\max } / 2$, is taken as 1 unit and according to this value the cell radius and RS coverage area circle radius values are found. Figure 3.9 is for propagation constant of 4 . As seen from the figure, if the RS power is 0.1 times the $\mathrm{CN}$ power, the area between two circles can almost be covered with RSs of one tier. If we assume that the distance between two RS is $\sqrt{3}$ times the radius of its coverage area and all the RSs are deployed on a circle, total number of RS required can be found as 17 . For such a case, from Table 3.4, the combined 2-hop spectral efficiency at the cell border is $2.47 \mathrm{bits} / \mathrm{sec} / \mathrm{Hz}$ and higher than the maximum attainable combined 3-hop spectral efficiency; thus only one tier of RSs can is enough. On the other hand, as seen from the Figure 3.10 , for $n=3$ case, even if the transmit power of RS is increased to 0.3 times the power of the $\mathrm{CN}$, only half of the $R 2$ is covered by the RS with the maximum spectral efficiency. So, there should be more than one tier of RSs in $R 2$. 


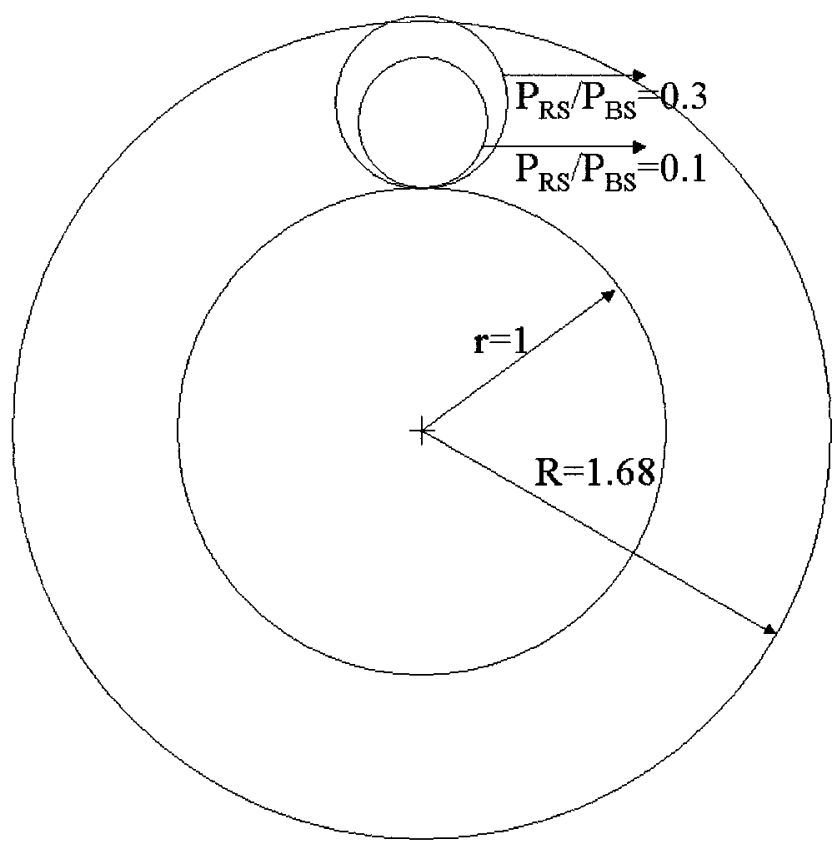

Figure 3.9: Cell structure for $n=4$.

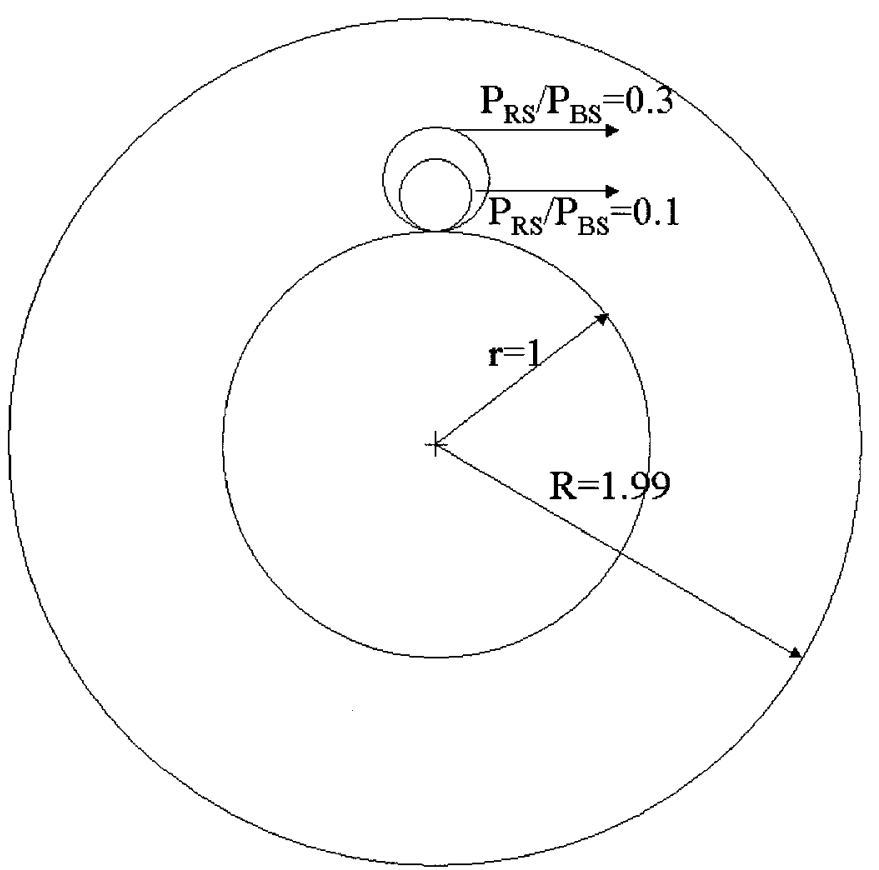

Figure 3.10: Cell structure for $n=3$. 
Figure 3.11 shows the relation between the number of RSs needed and the propagation constant for several values of the RS transmit power when $\mathrm{CN}$ transmit power is $10 \mathrm{~W}$. If the RS transmit power is increased, high data rate coverage area of the RS increases as well; accordingly the number of RSs needed decreases. Figure 3.11 also indicates that, the lower the propagation constant, the more the number of the RSs needed.

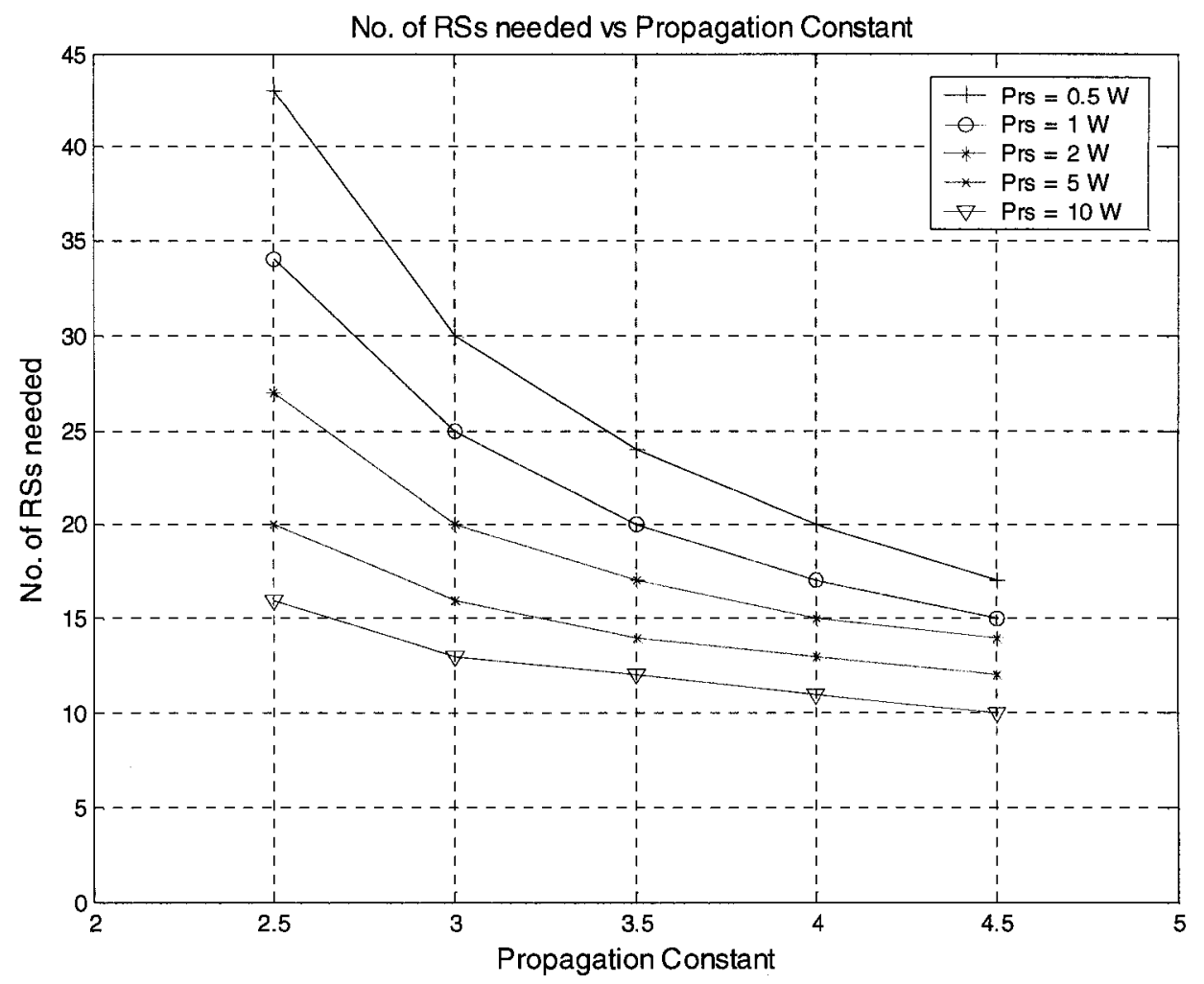

Figure 3.11: Relation between the number of RSs needed and propagation constant for several values of the RS transmit power (no shadowing, uniform interference power, $\left.\mathrm{P}_{\mathrm{CN}}=10 \mathrm{~W}\right)$. 


\section{6. $\quad$ Reusing Time Slots}

Each RS-to-RS link uses directional antennas for better performance. Therefore, two links that are not neighbors do not interfere with each other, enabling the reuse of the channels. In other words, not all of the channels links for the multi-hop communication are supposed to be orthogonal to each other.

To simplify the analysis odd indexed and even indexed links (indexes come from spectral efficiency indexes in Figure 3.1 can be grouped separately. At this point, none of the links in the same group (even indexed or odd indexed) create interference on each other. Furthermore, links in the same group can be re-arranged according to their spectral efficiencies; $c_{1}$ being the spectral efficiency of the link having the best spectral efficiency among the odd indexed links, $c_{3}$ being the spectral efficiency of the link having the second best spectral efficiency among the odd indexed links, and so on. Similarly, $c_{2}$ being the spectral efficiency of the link having the best spectral efficiency among the even indexed links, $c_{4}$ being the spectral efficiency of the link having the second best spectral efficiency among the odd indexed links, and so on. At this point, without loss of generality we may assume that number of hops, namely $n$, is an even integer. Therefore, it can be observed that $c_{1}>c_{3}>c_{5}>\ldots>c_{n-1}$ and $c_{2}>c_{4}>c_{6}>\ldots>c_{n}$ (equality is ignored for simplicity). The link having the spectral efficiency $c_{1}$ is the best link among the odd indexed links, so it will need the least transmission time, $T_{1}$. Since $c_{3}<c_{1}$, the link having the spectral efficiency $c_{3}$ will need more transmission time than the link 
having the spectral efficiency $c_{1}$. Those links do not create interference on each other, therefore, link having the spectral efficiency $c_{3}$ can transmit at $T_{1}$ and some additional time $T_{3}$. By the same logic, the link having the spectral efficiency $c_{5}$ can transmit at $T_{1}$, $T_{3}$, and some additional time $T_{5}$, and so on.

Similarly, the link having the spectral efficiency $c_{2}$ uses transmission time $T_{2}$, link having the spectral efficiency $c_{4}$ uses transmission time $T_{2}+T_{4}$, link having the spectral efficiency $c_{6}$ uses transmission time $T_{2}+T_{4}+T_{6}$, and so on. Similar to (3.5), following equalities can be derived,

$$
\begin{aligned}
& c_{1} * T_{1}=c_{3} *\left(T_{1}+T_{3}\right)=c_{5} *\left(T_{1}+T_{3}+T_{5}\right)=\ldots=c_{n-1} *\left(T_{1}+T_{3}+T_{5}+\ldots+T_{n-1}\right) \\
& =c_{2} * T_{2}=c_{4} *\left(T_{2}+T_{4}\right)=c_{6} *\left(T_{2}+T_{4}+T_{6}\right)=\ldots=c_{n} *\left(T_{2}+T_{4}+T_{6}+\ldots+T_{n}\right) .
\end{aligned}
$$

From those equations, following ones can be obtained:

$$
\begin{array}{ll}
T_{3}=c_{1}\left(\frac{1}{c_{3}}-\frac{1}{c_{1}}\right)^{*} T_{1} & T_{4}=c_{2}\left(\frac{1}{c_{4}}-\frac{1}{c_{2}}\right) * T_{2} \\
T_{5}=c_{1}\left(\frac{1}{c_{5}}-\frac{1}{c_{3}}\right) * T_{1} & T_{6}=c_{2}\left(\frac{1}{c_{6}}-\frac{1}{c_{4}}\right) * T_{2} \\
T_{2^{*} m+1}=c_{1}\left(\frac{1}{c_{2^{*} m+1}}-\frac{1}{c_{2^{*} m-1}}\right) * T_{1} & T_{2^{*} m}=c_{2}\left(\frac{1}{c_{2^{*} m}}-\frac{1}{c_{2^{*} m-2}}\right) * T_{2} .
\end{array}
$$




$$
\begin{aligned}
& \frac{\text { Data Rate }_{\mathrm{MH}}}{\text { Data Rate }_{\mathrm{SH}}}=\frac{T}{T_{1}+T_{2}+T_{3}+\ldots+T_{n}} \\
& =\frac{T}{T_{1}+T_{2}+c_{1}\left(\frac{1}{c_{3}}-\frac{1}{c_{1}}\right) * T_{1}+c_{2}\left(\frac{1}{c_{4}}-\frac{1}{c_{2}}\right) * T_{2}+\ldots+c_{1}\left(\frac{1}{c_{n-1}}-\frac{1}{c_{n-3}}\right) * T_{1}+c_{2}\left(\frac{1}{c_{n}}-\frac{1}{c_{n-2}}\right) * T_{2}} \\
& =\frac{T}{\frac{c_{1}}{c_{n-1}} * T_{1}+\frac{c_{2}}{c_{n}} * T_{2}}=\frac{\frac{T}{T_{1}}}{\frac{c_{1}}{c_{n-1}}+\frac{c_{2}}{c_{n}} * \frac{T_{2}}{T_{1}}}=\frac{\frac{c_{1}}{b}}{\frac{c_{1}}{c_{n-1}}+\frac{c_{2}}{c_{n}} * \frac{c_{1}}{c_{2}}}=\frac{\frac{1}{b}}{\frac{1}{c_{n-1}}+\frac{1}{c_{n}}} \\
& =\frac{\frac{1}{\frac{1}{c_{n-1}}+\frac{1}{c_{n}}}}{b}=\frac{c_{c}}{b} \text {, }
\end{aligned}
$$

where

$$
\begin{aligned}
& c_{n-1}=\min \left\{a_{1}, a_{3}, a_{5}, \ldots a_{n-1}\right\} \\
& c_{n}=\min \left\{a_{2}, a_{4}, a_{6}, \ldots a_{n}\right\}
\end{aligned} \quad \text { and } \quad c_{c}=\frac{1}{\frac{1}{c_{n-1}}+\frac{1}{c_{n}}}
$$

Equation (3.36) is very similar to (3.17) for 2-hop case; $c_{c}$ can be thought as the combined spectral efficiency for the $n$-hop communication where the time slots are reused. Previously, it is found that, if the time slots of the composite multi-hop link are orthogonal to each other, combined spectral efficiency decreases as the number of hops increases. On the other hand, if the time slots of the composite multi-hop link are reused as explained above, then the combined spectral efficiency is independent of the number 
of hops; the combined spectral efficiency is always equal to that of a 2-hop communication with orthogonal time slots. It should be noted that, both entirely orthogonal and reusing the time slots models analyzed in this chapter are extreme cases. For the best performance, an optimum combination of these two cases can be used. 


\section{CHAPTER 4: SIMULATION MODEL}

This chapter introduces the simulation algorithm. The first section discusses the propagation model, followed by a section on environmental parameters used in the simulation. Then, adaptive modulation and coding (AMC), which enables the efficient utilization of the spectrum, is briefly explained. Finally the simulation algorithm is described by figures.

\subsection{Propagation Model}

The most basic model of radio wave propagation is so called free space radio wave propagation. In this model, radio waves emanate from a point source of radio energy, traveling in all directions in a straight line, filling the entire spherical volume of space with radio energy that varies in strength with a $1 / d^{2}$ rule, where $d$ is the distance from the radio source. However, real world radio propagation rarely follows this simple model. Due to several reasons (e.g. reflection, diffraction, scattering) radio channels change in a statistical way. In order to take these affects into consideration following propagation model is used in this research:

$$
P_{r}=P_{t} \frac{G}{\overline{P L}} X_{\sigma}
$$

where 
$P_{r}:$ Received power

$P_{t}:$ Transmit power

$G$ : Combined antenna gain of the transmitter and the receiver

$X_{\sigma}$ : Log-normally distributed random variable with $0(\mathrm{~dB})$ mean, $\sigma(\mathrm{dB})$ standard deviation

$\overline{P L}:$ Average pathloss

Average pathloss, $\overline{P L}$, can be defined as follows:

$$
\overline{P L}=\left(\frac{4 \pi d_{0} f}{c}\right)^{2}\left(\frac{d_{T R}}{d_{0}}\right)^{n},
$$

where

$d_{0}:$ Close-in reference distance

$f$ : Carrier frequency

$c$ : Speed of light

$d_{T R}:$ Distance between the transmitter and the receiver antennas

$n$ : Propagation exponent

\subsection{Environment and Parameters Assumptions}

The following environment and parameter assumptions are used in the simulation algorithms. They are typical data widely used in simulating cellular networks. 
- Pathloss propagation exponent: $n=3.5$ for CN-MS and RS-MS links; $n=2.4$ for CN-RS links.

- Lognormal shadowing with a standard deviation: $\sigma=8 \mathrm{~dB}$ for CN-MS and RS-MS links; $\sigma=3 \mathrm{~dB}$ for $\mathrm{CN}-\mathrm{RS}$ links

- Simulation area: hexagonal cells with the cell radius $R=500 \mathrm{~m}, 1000 \mathrm{~m}$; 1-cell cluster. Seven clusters are investigated, i.e., interference originates from only the first-tier cells.

- RF carrier: $f=5 \mathrm{GHz}$

- Transmission bandwidth: $W=50 \mathrm{MHz}$

- Thermal noise with a noise figure: $F_{N}=8 \mathrm{~dB}$

- Omni-directional antennas (with $3 \mathrm{~dB}$ gain) for the CN-MS and RS-MS links; directional antennas (with $20 \mathrm{~dB}$ gain) for the CN-RS links

- No power control

- CN transmit power: $P_{C N}=10 \mathrm{~W} ; \mathrm{RS}$ transmit power: $P_{R S}=1 \mathrm{~W}$

- Downlink scenario only

\subsection{Adaptive Modulation and Coding}

As mentioned before, the main purpose of the next generation cellular systems is to provide high data rate coverage. In order to realize that goal, in addition to the changes in the network structure, the available spectrum must be utilized effectively. Adaptive 
Modulation and Coding (AMC) technique has the potential to increase the spectrum efficiency [20]. Transmission techniques that do not change according to the varying characteristics of the link require fixed link margin to provide the tolerable performance in deep fades. So, these systems are planned for the worst-case channel systems. The core idea behind the AMC is to dynamically adapt the modulation and coding scheme according to the instantaneous propagation conditions and interference scenarios, i.e. received SINR values.

Basically, the combinations of three modulation schemes (QPSK, 16-QAM, 64-QAM) and five code rates $(1 / 2,2 / 3,3 / 4,7 / 8$ and 1$)$ are considered in this research. Table 3.2 shows the relation between the spectral efficiency and the SINR.

\subsection{Simulation Algorithm}

Simulations are done in order to support the analytical results found in the previous chapter. In the simulations, the performances of non-relaying and relaying cases are compared. 2000 MSs are placed randomly across the cell to get the spectral efficiency for every point in the cell. Frequency reuse of one is used in the system, i.e. the cluster size is one and all available channels can be used in a cell. Since same channel is not used more than once in a cell, at any moment, there is only one interferer from a cell. It is assumed that, the users (in different cells) using the same channels are located at the same position with respect to the position of the CNs of their cells (as shown in Figure 4.2). For the non-relaying case, all MSs receive signals from the $\mathrm{CN}$. In this case, interference comes 
only from the first-tier CNs. Figure 4.1 shows the intended (red arrow) and the interference (black arrows) signals for an arbitrary user in the cell.

Following formula can be used to calculate the total interference power:

$$
P_{I}=P_{1}+P_{2}+P_{3}+P_{4}+P_{5}+P_{6}
$$

where $P_{1}, \ldots, P_{6}$ are the interference powers from the first-tier CNs.

The noise power is calculated according to the following formula:

$$
P_{N}=K_{b} \times T \times W \times F_{N}=1.306 \times 10^{-12} \text { watts, }
$$

where

$K_{b}$ : Boltzmann's constant $(1.38 \times 10-23$ Joules/Kelvin $)$

$T:$ System temperature $(300 \mathrm{~K})$

$W$ : Transmission bandwidth $(50 \mathrm{MHz})$

$F_{N}:$ Noise figure $(6.31=8 \mathrm{~dB})$.

Then, if $P_{s}$ is assumed to be the intended received signal power, SINR can be written as follows,

$$
S I N R=\frac{P_{S}}{I S F \times P_{I}+P_{N}},
$$

where 
ISF : Interference Suppression Factor that takes values between 0 (all interference is suppressed) and 1 (no interference suppression is done). The frequency reuse factor used in the simulations is 1 , which leads to a high level of aggregate interference in the cell. Therefore, the system will need some interference reduction scheme to be able to work properly. In this research $I S F$ is taken as 0.5 , meaning half of the interference coming from interferers is suppressed.

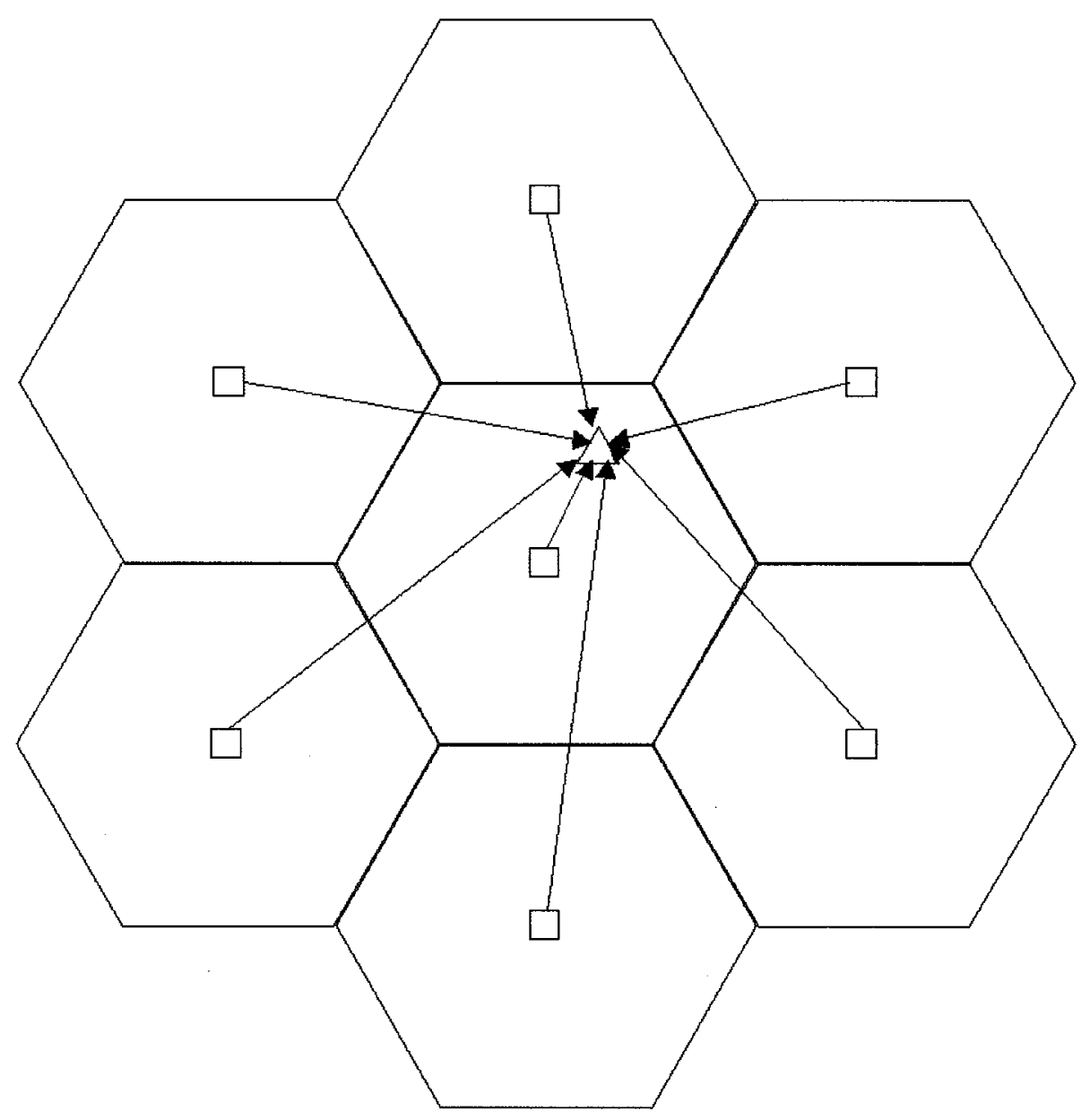

Figure 4.1: Intended signal and interferers for a MS. 
For the relaying case, first, RSs are placed around the CNs. The positions of RSs are found in Table 3.4; for $500 \mathrm{~m}$ cell radius, propagation constant 3.5, $\mathrm{CN}$ transmit power 10 watts and RS transmit power 1 watt, the CN-RS distance is found as $338 \mathrm{~m}$. The received signal can be either from $\mathrm{CN}$ or RS depending on the MS position and the channel conditions. If the signal comes from the $\mathrm{CN}$, the scenario is same as the nonrelaying case; as shown in Figure 4.2, interferers are the first-tier CNs and (4.5) can be used to find the SINR value for the MS. If the signal comes from one of the RSs, in addition to the RS-MS link, one more link, namely CN-RS link, is needed to complete the composite 2-hop link. As explained earlier, the time slots used by these two links are orthogonal to each other. Therefore, following situation occurs:

- At time slot $T_{1}$, the bandwidth available to the composite 2-hop link is used by the CN-RS link; during $T_{1}$ the RS functions as a receiver only. Since the origin of the signal is the $\mathrm{CN}$, the interferers are the first-tier CNs. According to the intended signal, interference, and noise power levels, the SINR (and spectral efficiency) of this link is obtained.

- At time slot $T_{2}$, the bandwidth available to the composite 2-hop link is used by the RS-MS link; during $T_{2}$ the RS functions as a transmitter only. Since the origin of the signal is the RS, the interferers are the co-channel RSs from the first-tier cells. Figure 4.3 shows the intended (red arrow) and the interference (black arrows) signals for this case. Similarly, according to the intended signal, interference, and noise power levels, the SINR (and spectral efficiency) of this 
link is obtained. Using (3.20) the combined spectral efficiency of the composite 2-hop link is found.

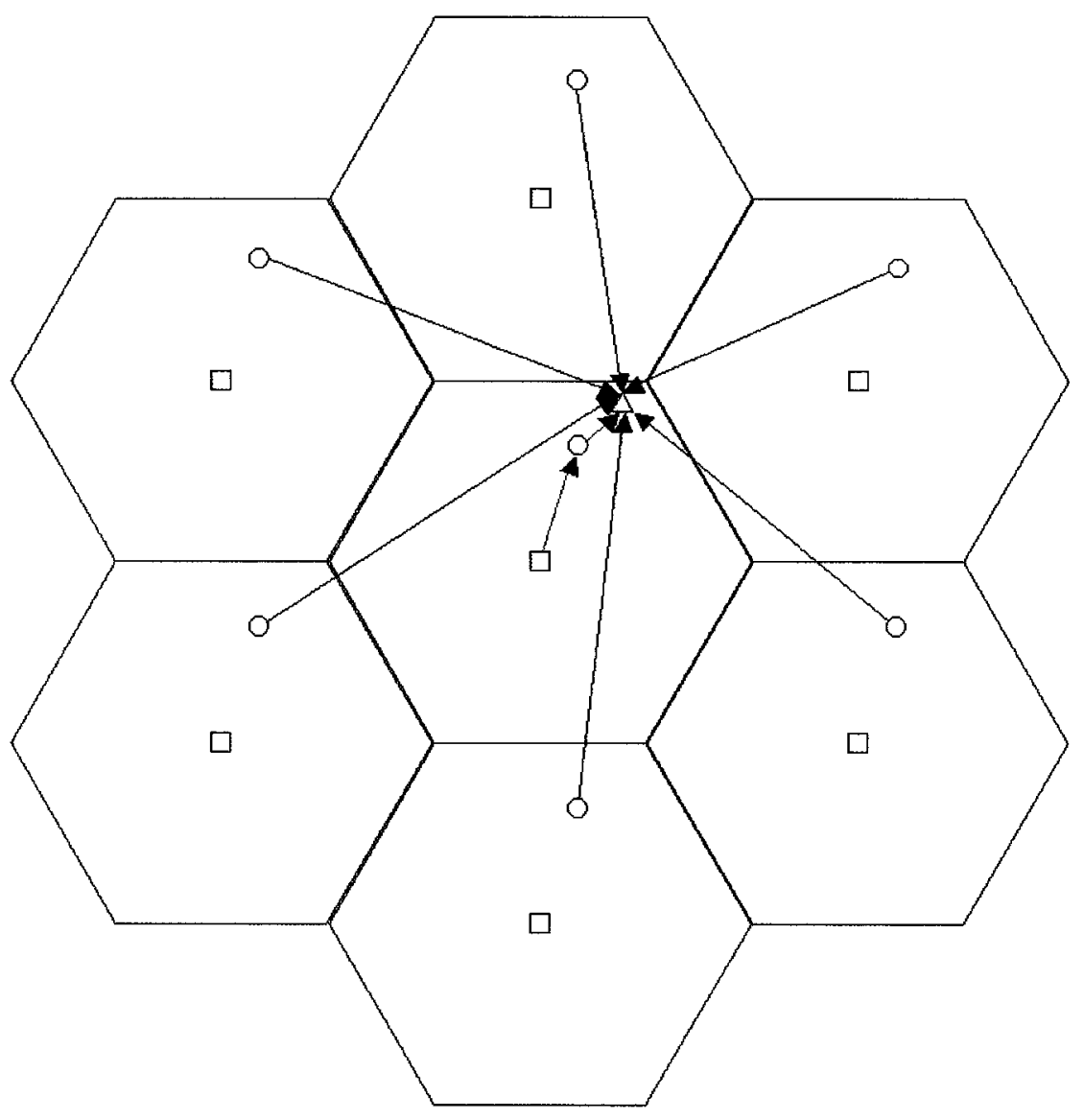

Figure 4.2: Intended signal and interferers for a MS using relay assistance. 


\subsection{Simulation Flowchart}

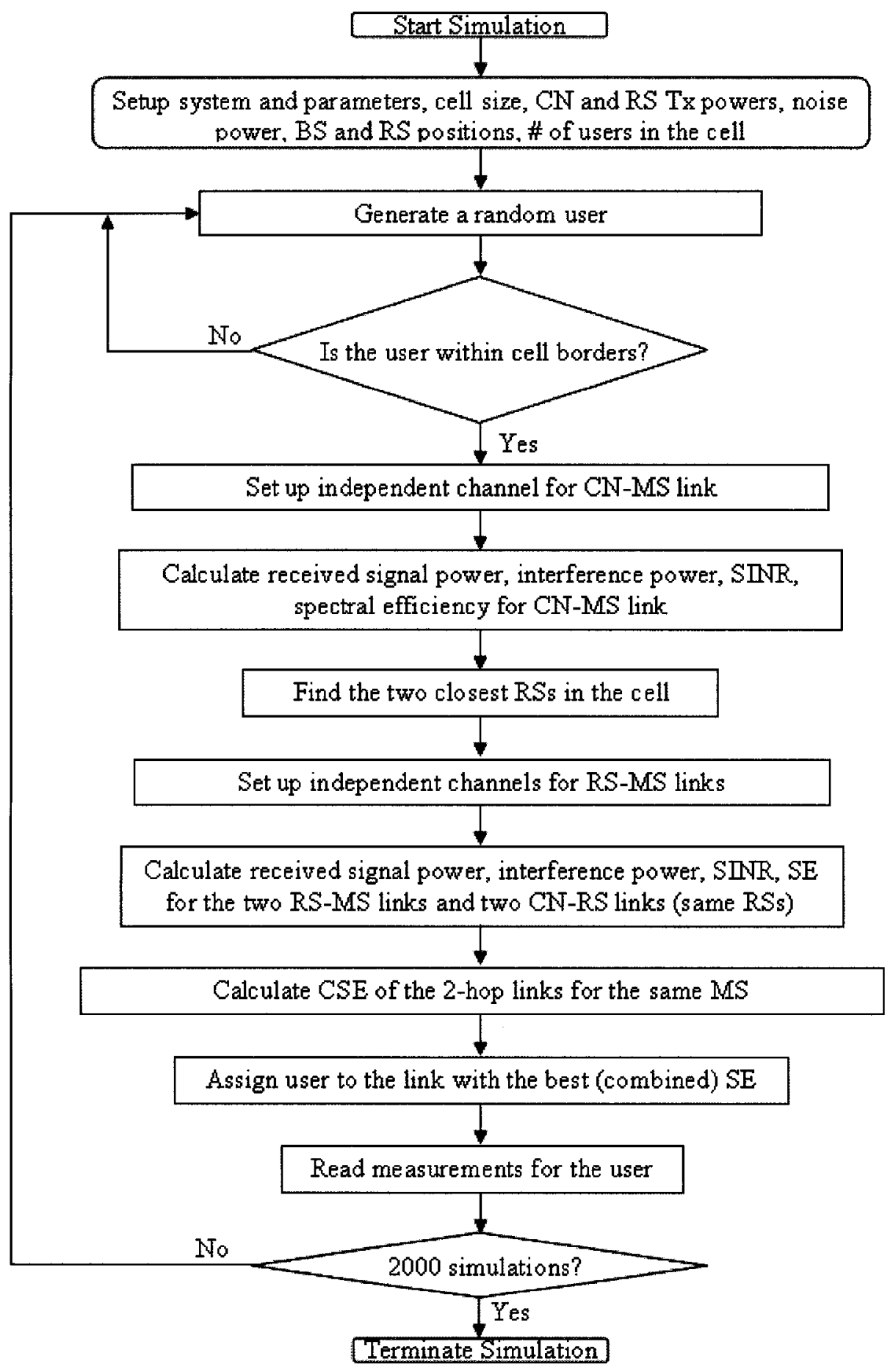

Figure 4.3: Simulation flowchart. 


\section{CHAPTER 5: $\quad$ SIMULATION RESULTS}

This chapter presents the simulation results for the system where the first tier RSs are deployed. The main objective of the simulations is to support the previously developed analytical results. In addition, potential coverage extension and throughput increase benefits of relay assistance is analyzed as well.

In the first section, the spectral efficiency distribution of MSs is shown for both nonrelaying and relaying cases. Then, average spectral efficiency and outage for nonrelaying and relaying cases are compared. Finally, cumulative distribution function (cdf) curves are shown for the performance comparison.

\subsection{Spectral Efficiency Distribution}

In this section, the simulation results for the spectral efficiency distribution of the nonrelaying and relaying cases are presented. Figures 5.1-5.5 demonstrate the spectral efficiency distribution for non-relaying and relaying $(6,18,30$, and 50 RSs around the $\mathrm{CN}$ ) cases where cell radius is $500 \mathrm{~m}$. Similarly, Figures 5.6-5.10 show the spectral efficiency distribution for non-relaying and relaying $(6,18,30$, and $50 \mathrm{RSs}$ around the $\mathrm{CN}$ ) cases where cell radius is $1000 \mathrm{~m}$. The red stars in the figures for the relaying cases represent the RS locations in the cell. In the figures black points denote the MSs having spectral efficiency values less than $1 \mathrm{bits} / \mathrm{sec} / \mathrm{Hz}$, i.e. outage points. The outage problem is very severe (especially for the $1000 \mathrm{~m}$ case) for both cases when relay assistance is not 
used. As 6 RSs are deployed around the CN (at a distance of $338 \mathrm{~m}$ and $676 \mathrm{~m}$ for $500 \mathrm{~m}$ cell and $1000 \mathrm{~m}$ cell, respectively), outage can be reduced significantly. However, in order to provide the high data rate coverage $6 \mathrm{RSs}$ are not enough; in fact $30 \mathrm{RSs}$ are required. It is worth mentioning that, if the number of RSs is increased further than 30 , the performance improvement is almost negligible. Another point to be noticed is that, in some of the figures (especially in Figure 5.2), the spectral efficiency distribution is not symmetrical with respect to the axes. The reason for this behavior is that, the CN-RS links have also lognormal shadowing effect, and hence some of the links (links between the $\mathrm{CN}$ and $\mathrm{RSs}$ located at $180^{\circ}$ and $240^{\circ}$ ) do not have very good spectral efficiency, which affects the combined spectral efficiency for the users in those RSs' coverage area.

For the Figures 5.1 to 5.10 , different colors stand for different spectral efficiency levels:

\begin{tabular}{|c|c|}
\hline Red & $: S E>=3.0$ \\
\hline \multirow[t]{2}{*}{ Magenta } & $: 3.0>S E \geq 2.75$ \\
\hline & $2.75>S E \geq 2.5$ \\
\hline Green & $: 2.5>S E \geq 2.0$ \\
\hline Cyan & $: 2.0>S E \geq 1.5$ \\
\hline Blue & $: 1.5>S E \geq 1$ \\
\hline Black & $: S E<1$ \\
\hline
\end{tabular}




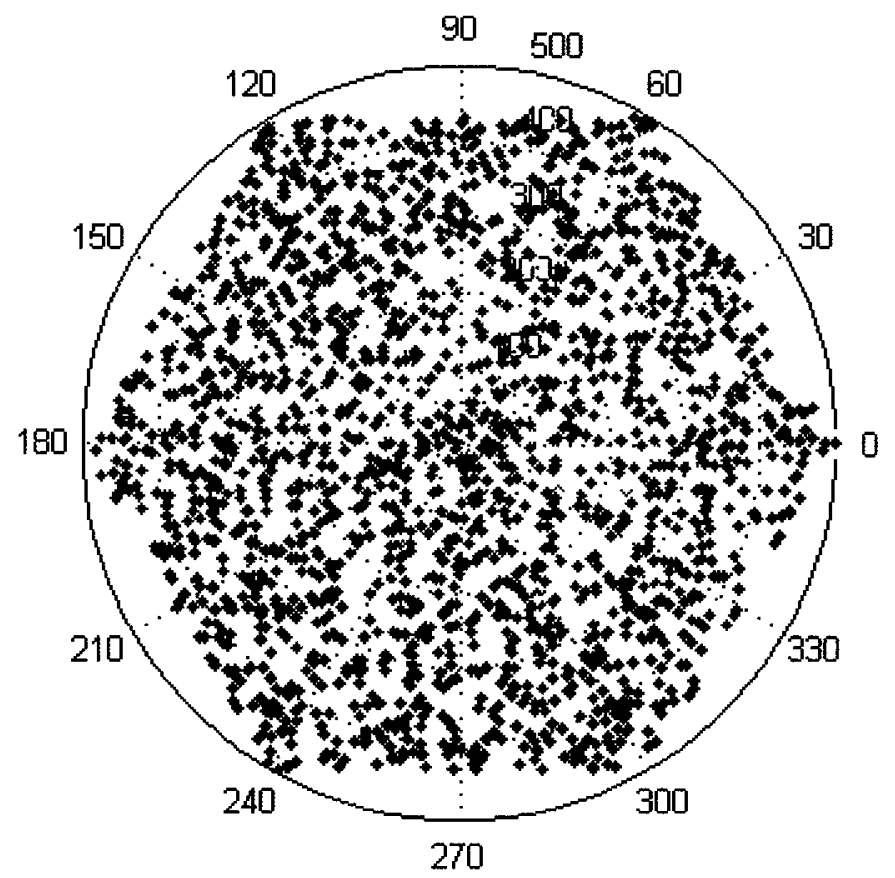

Figure 5.1: Spectral efficiency distribution for non-relaying case $(\mathrm{R}=500 \mathrm{~m})$.

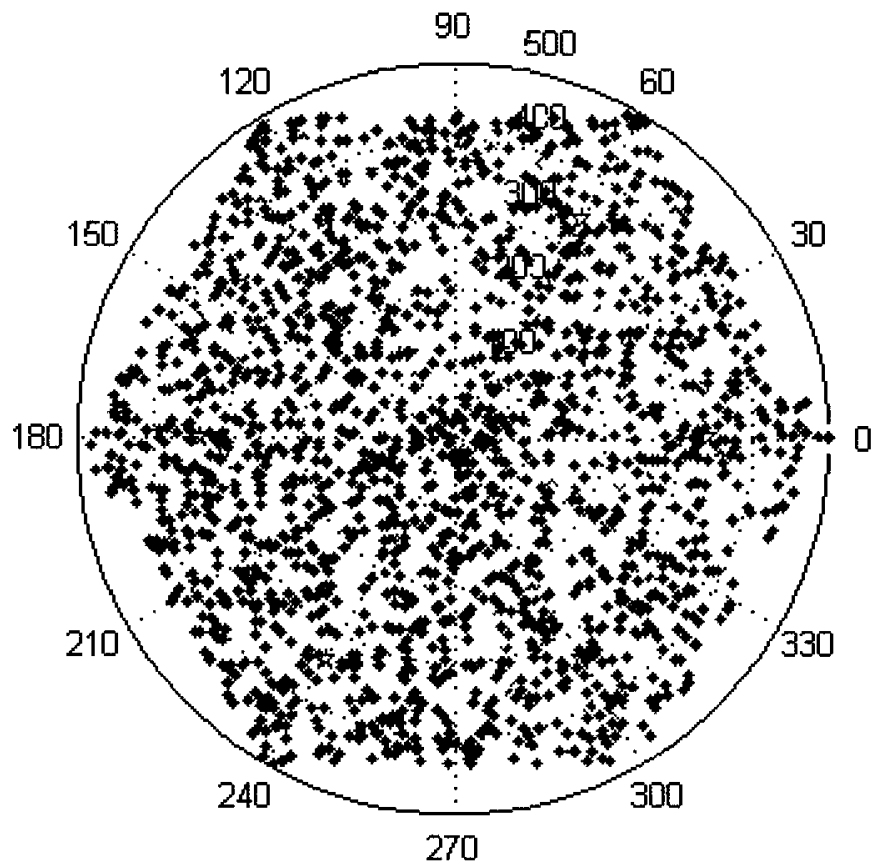

Figure 5.2: Spectral efficiency distribution for $6 \mathrm{RS}$ case $(\mathrm{R}=500 \mathrm{~m})$. 


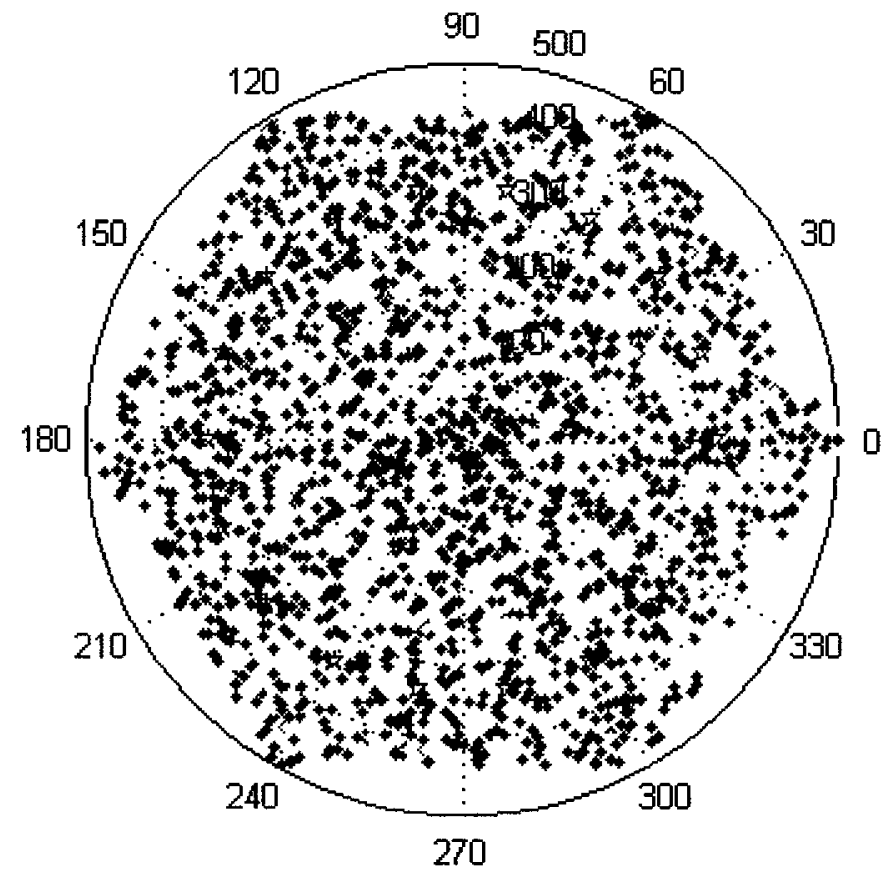

Figure 5.3: Spectral efficiency distribution for $18 \mathrm{RS}$ case $(\mathrm{R}=500 \mathrm{~m})$.

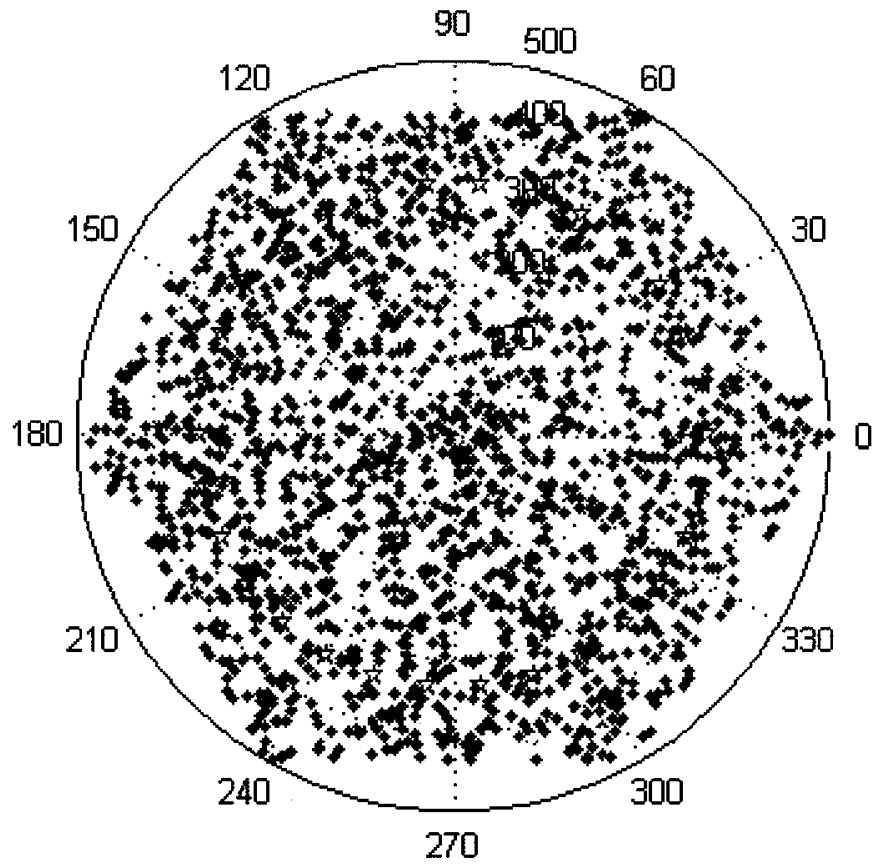

Figure 5.4: Spectral efficiency distribution for $30 \mathrm{RS}$ case $(\mathrm{R}=500 \mathrm{~m})$. 


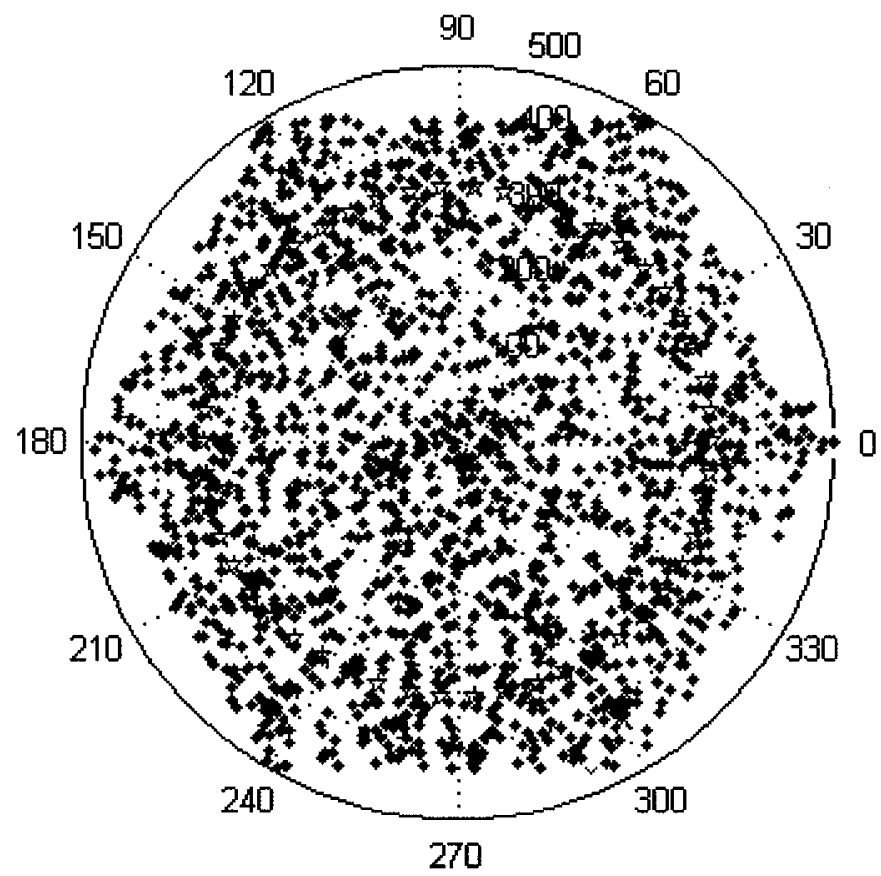

Figure 5.5: Spectral efficiency distribution for $50 \mathrm{RS}$ case $(\mathrm{R}=500 \mathrm{~m})$.

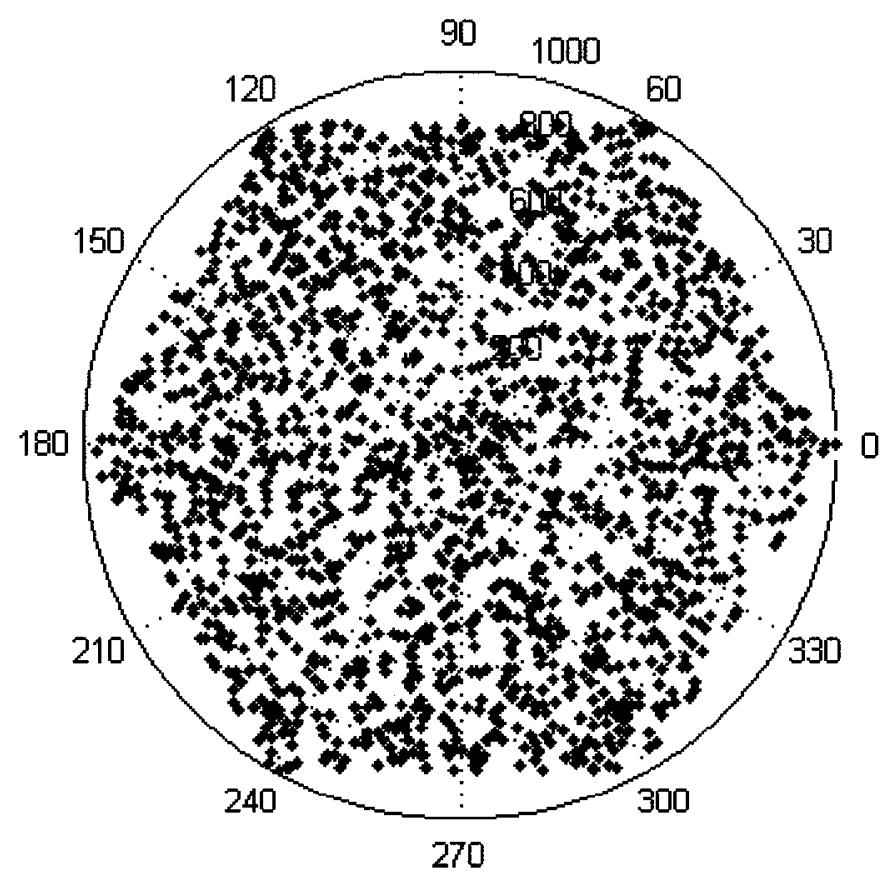

Figure 5.6: Spectral efficiency distribution for no $R S$ case $(R=1000 \mathrm{~m})$. 


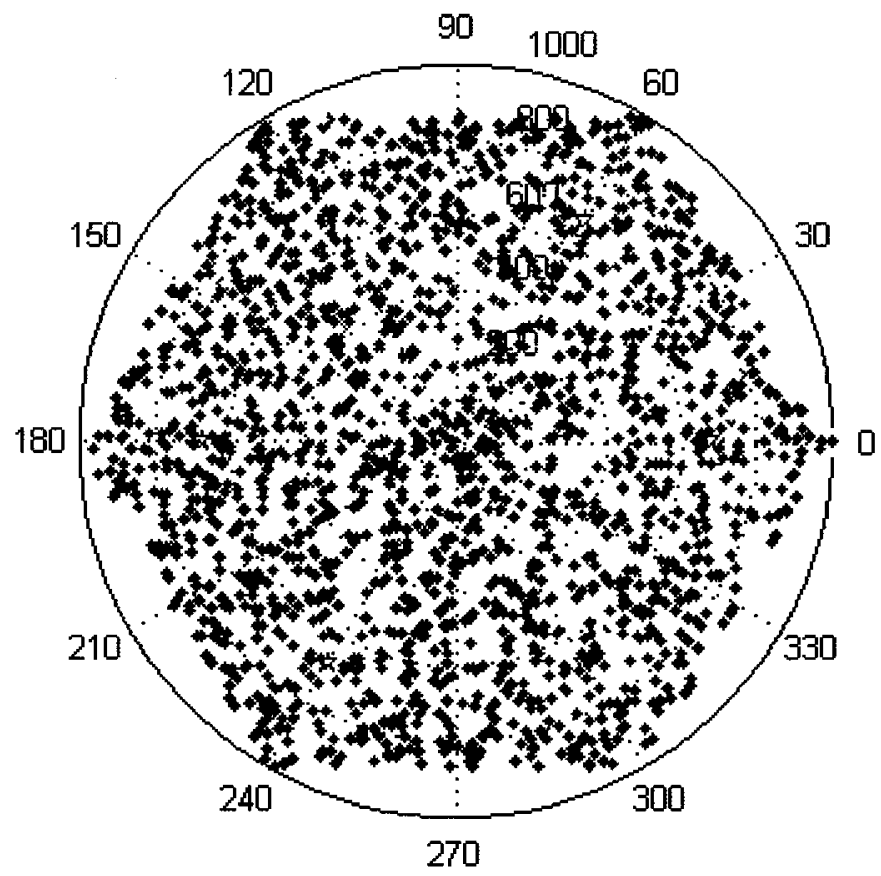

Figure 5.7: Spectral efficiency distribution for $6 \mathrm{RS}$ case $(\mathrm{R}=1000 \mathrm{~m})$.

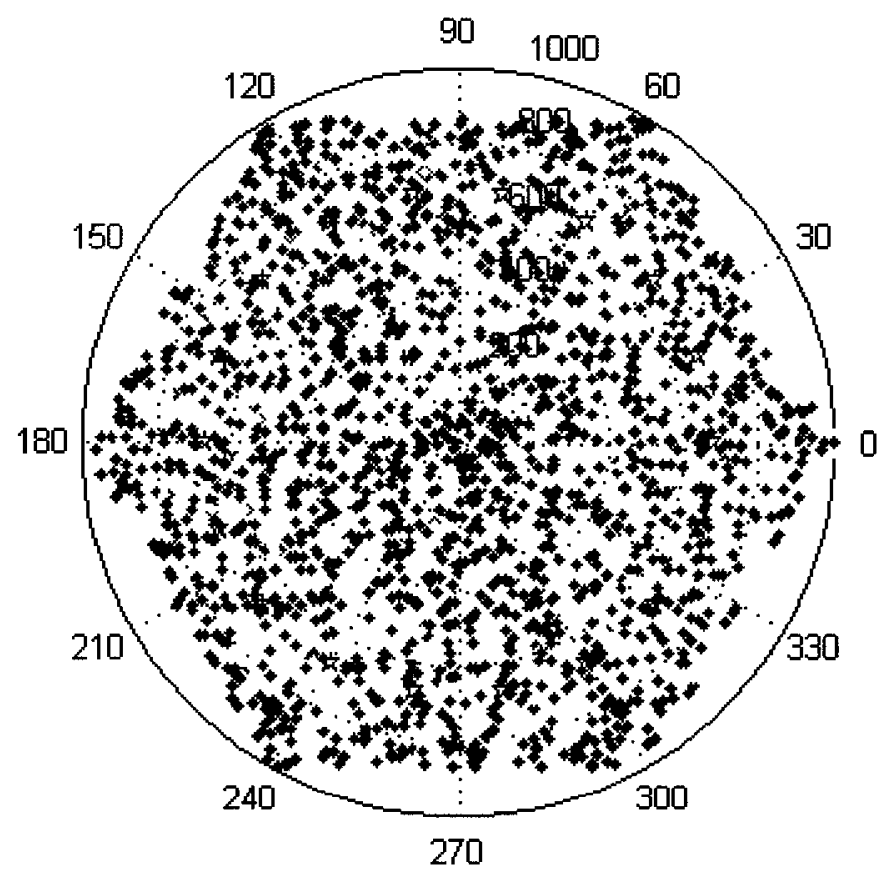

Figure 5.8: Spectral efficiency distribution for $18 \mathrm{RS}$ case $(\mathrm{R}=1000 \mathrm{~m})$. 


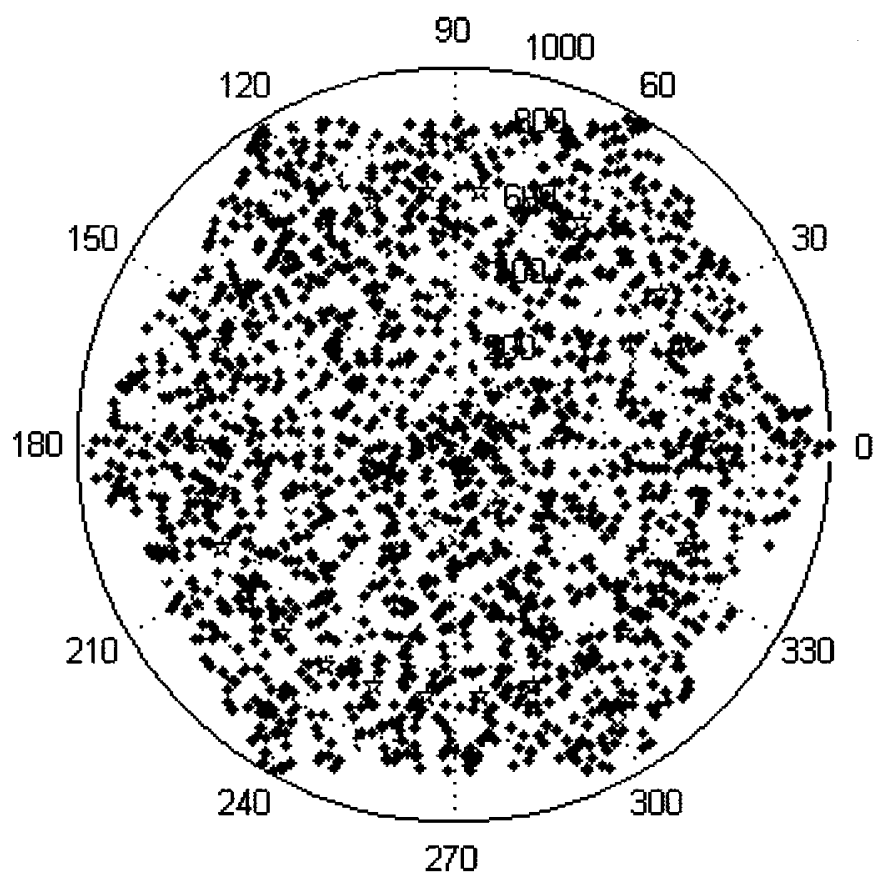

Figure 5.9: Spectral efficiency distribution for $30 \mathrm{RS}$ case $(\mathrm{R}=1000 \mathrm{~m})$.

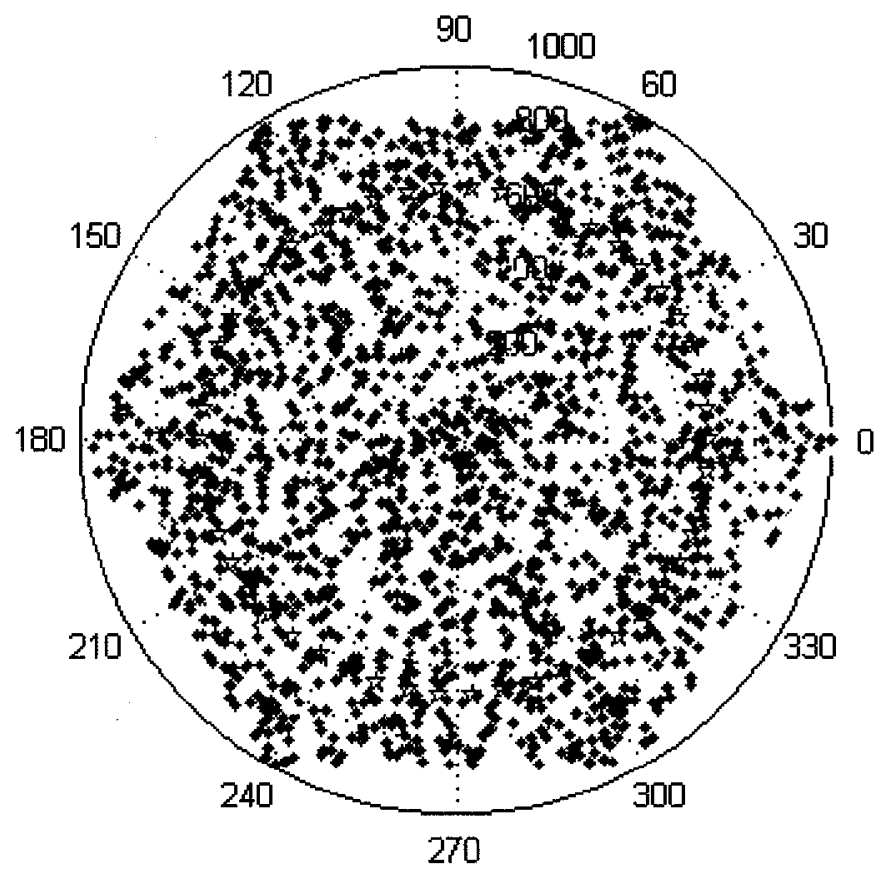

Figure 5.10: Spectral efficiency distribution for $50 \mathrm{RS}$ case $(\mathrm{R}=1000 \mathrm{~m})$. 


\subsection{Coverage at Various Spectral Efficiency Levels with respect to Number of RSs}

Figures 5.11 and 5.12 illustrate the coverage performance, i.e. the probability of combined spectral efficiency being greater than the values given in the horizontal axis, for $500 \mathrm{~m}$ and $1000 \mathrm{~m}$ cells, respectively. Figure 5.11 indicates that if the relaying assistance is used in the system, even for the 6 RS case, the performance gain is substantially improved. However, in order to realize the high data rate coverage goal, the required number of RSs is moderately high, i.e. around $30(67 \%$ of the users have spectral efficiency values greater than or equal to $3 \mathrm{bits} / \mathrm{sec} / \mathrm{Hz}$ ). It is also observed that, if the number of RSs is increased further than 30 , the performance gain is insignificant. Moreover, maximum attainable spectral efficiency for 3-hop communication is 2 bits $/ \mathrm{sec} / \mathrm{Hz}$; since $96 \%$ of the users already have spectral efficiency values greater than or equal to $2 \mathrm{bits} / \mathrm{sec} / \mathrm{Hz}$ via 2-hop communication, there is no need for 3-hop communication.

Figure 5.12 shows that, even though 2-hop communication helps the outage problem of the $1000 \mathrm{~m}$ case, the high data rate coverage problem is not solved (for 50 RS case $43 \%$ of the users have spectral efficiency values greater than or equal to $3 \mathrm{bits} / \mathrm{sec} / \mathrm{Hz}$ ). It is observed that increasing the number of RSs from 30 to 50 does not improve the performance as expected; as a result, instead of adding more RSs to the first tier, a second tier of RSs should be deployed around the CN.

Some additional comments can be done on Figures 5.11 and 5.12. Previously, it is shown that for 2-hop communication the best spectral efficiency level is $3 \mathrm{bits} / \mathrm{sec} / \mathrm{Hz}$. In 
other words, for the relaying case, if the spectral efficiency of the user is greater than 3 bits/sec/Hz, the user is assigned directly to the $\mathrm{CN}$, i.e. single-hop communication. Therefore, the number of users having spectral efficiency values greater than 3 bits/sec/Hz must be same for both non-relaying and relaying cases, since those users will use single-hop communication for either cases; in Figures 5.11 and 5.12 coverage at the spectral efficiency levels greater than $3 \mathrm{bits} / \mathrm{sec} / \mathrm{Hz}$ (since in our case the AMC levels are discrete, this corresponds to the levels greater than or equal to $3.5 \mathrm{bits} / \mathrm{sec} / \mathrm{Hz}$, which is the next AMC level) is almost same for all cases; the small variations are due to the random lognormal shadowing. This threshold level depends on the maximum available spectral efficiency level of the system and the next AMC level greater than the half of the maximum available spectral efficiency level of the system. Even though the threshold value is independent from the cell size and the RS transmit power, these two factors have an important role in determining the vertical position of the threshold level; if the probability of the usage of the spectral efficiency levels greater or equal to $3.5 \mathrm{bits} / \mathrm{sec} / \mathrm{Hz}$ increases, the vertical position of the threshold level increases as well. On the other hand, coverage at the spectral efficiency level of $3 \mathrm{bits} / \mathrm{sec} / \mathrm{Hz}$ is different due to the fact that relaying may improve the spectral efficiency levels of some users and introduce new users with spectral efficiency level of $3 \mathrm{bits} / \mathrm{sec} / \mathrm{Hz}$ to the system. 


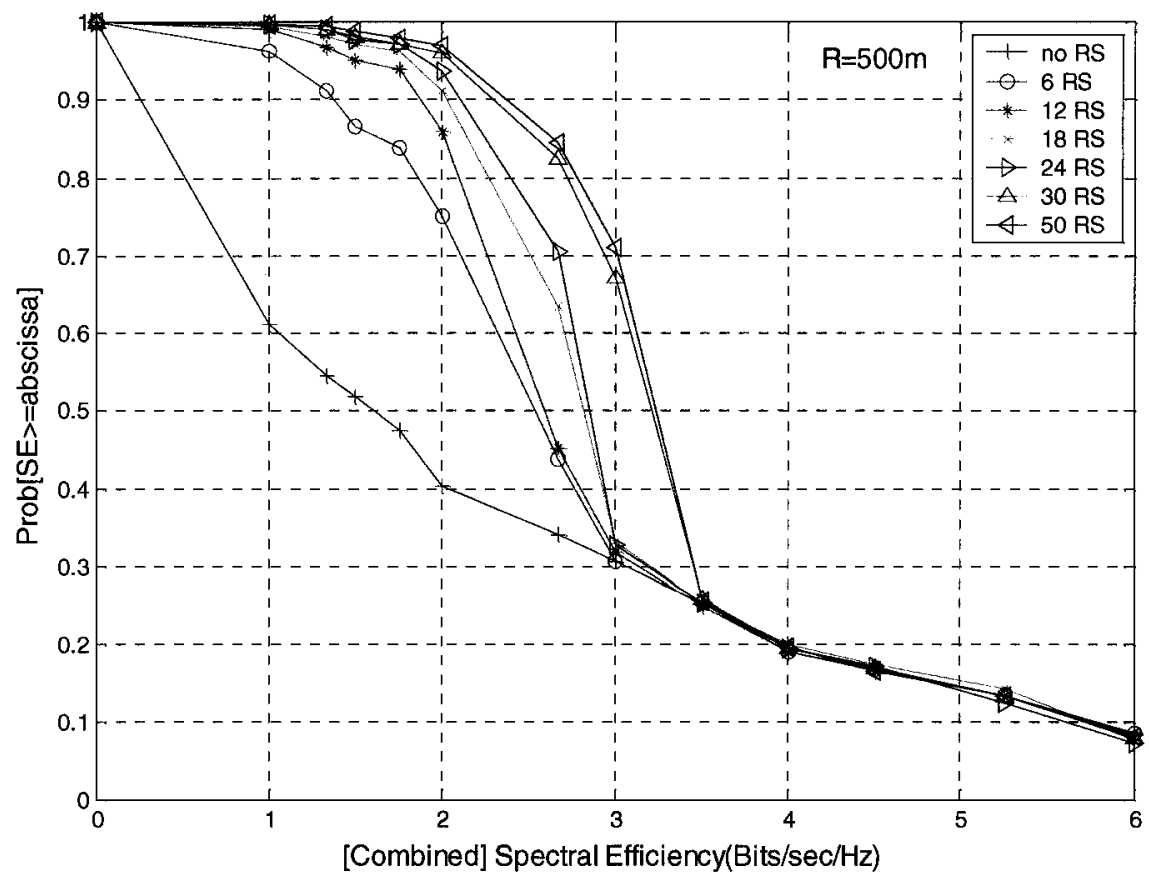

Figure 5.11: Coverage at various SE levels wrt no. of RSs for $500 \mathrm{~m}\left(\mathrm{P}_{\mathrm{RS}}=1 \mathrm{~W}\right.$, $\left.\mathrm{P}_{\mathrm{CN}}=10 \mathrm{~W}\right)$.

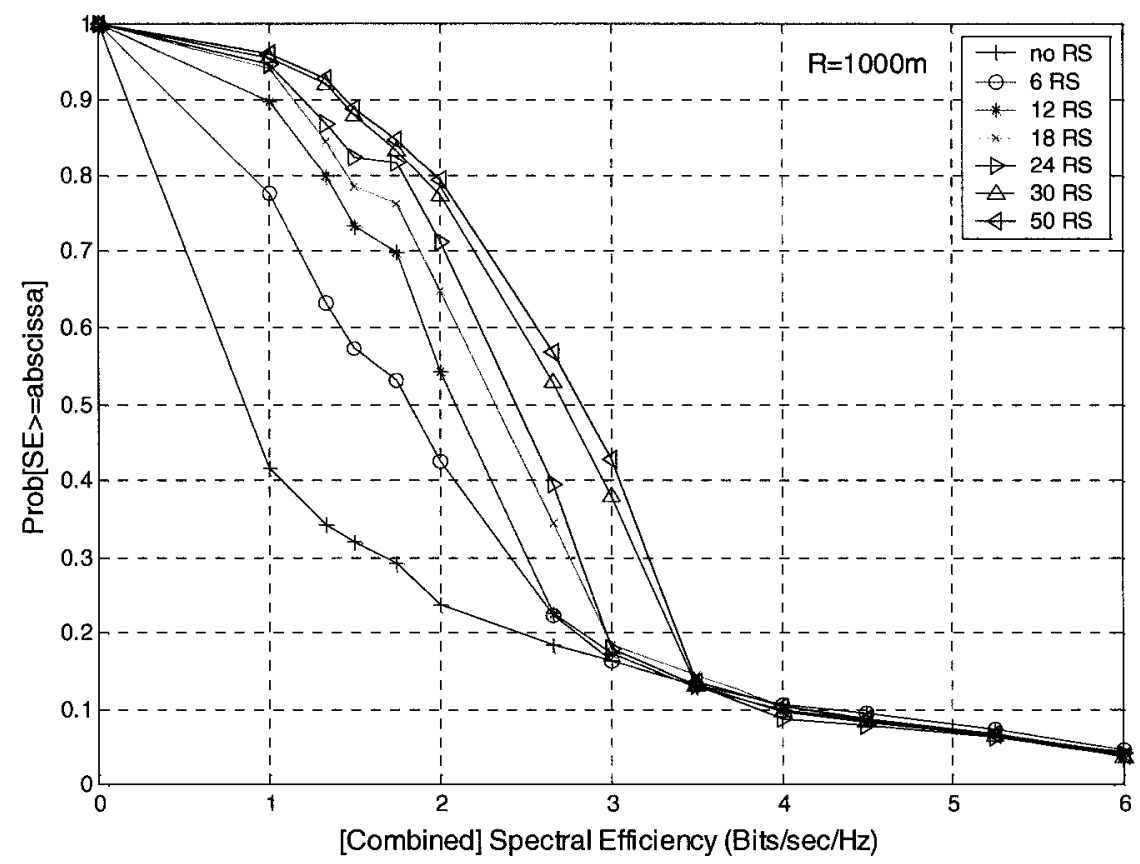

Figure 5.12: Coverage at various $\mathrm{SE}$ levels wrt no. of RSs for $1000 \mathrm{~m}\left(\mathrm{P}_{\mathrm{RS}}=1 \mathrm{~W}\right.$, $\left.\mathrm{P}_{\mathrm{CN}}=10 \mathrm{~W}\right)$. 


\subsection{Average Spectral Efficiency for Non-relaying and Relaying Cases}

Figures $5.13 \& 5.14$ depict the average spectral efficiency for $500 \mathrm{~m}$ and $1000 \mathrm{~m}$ cell radii, respectively. From the figures we can see that, even for the 6 RS case, average spectral efficiency is increased when the relaying assistance is used. Figure 5.13 indicates that, if 6 RSs are deployed around the $\mathrm{CN}$, the average spectral efficiency improvement is $0.92 \mathrm{bits} / \mathrm{sec} / \mathrm{Hz}$, which corresponds to a $48 \%$ increase $(1.9 \rightarrow 2.82)$. If the number of RSs is increased to 30 , the average spectral efficiency improvement is $1.39 \mathrm{bits} / \mathrm{sec} / \mathrm{Hz}$, which corresponds to a $73 \%$ increase $(1.9 \rightarrow 3.29)$. It is also observed that, when 50 RSs are used, the average spectral efficiency becomes $3.31 \mathrm{bits} / \mathrm{sec} / \mathrm{Hz}$; only $0.6 \%$ more than the 30 RS case. Furthermore, the result for 30 RS case is compared to that of the ideal ${ }^{3} 2-$ hop case, where the MSs that use 2-hop communication assumed to have spectral efficiency of $3 \mathrm{bits} / \mathrm{sec} / \mathrm{Hz}$, i.e. maximum attainable 2-hop combined spectral efficiency. The results for both cases are very close (6\% difference).

Similarly, for $1000 \mathrm{~m}$ cell size, if $6 \mathrm{RSs}$ are deployed around the $\mathrm{CN}$, the average spectral efficiency improvement is $0.77 \mathrm{bits} / \mathrm{sec} / \mathrm{Hz}$, which corresponds to a $66 \%$ increase $(1.15 \rightarrow 1.92)$. If the number of RSs is increased to 30 , the average spectral efficiency improvement is $1.48 \mathrm{bits} / \mathrm{sec} / \mathrm{Hz}$, which corresponds to a $129 \%$ increase $(1.15 \rightarrow 2.63)$. Although the average spectral efficiency is increased substantially, the difference against the ideal 2-hop case is significant (20\%), which again shows that for $1000 \mathrm{~m}$ cell size, it is better to use 2 tiers of RSs.

\footnotetext{
${ }^{3}$ Detials are given in the Appendix C.
} 


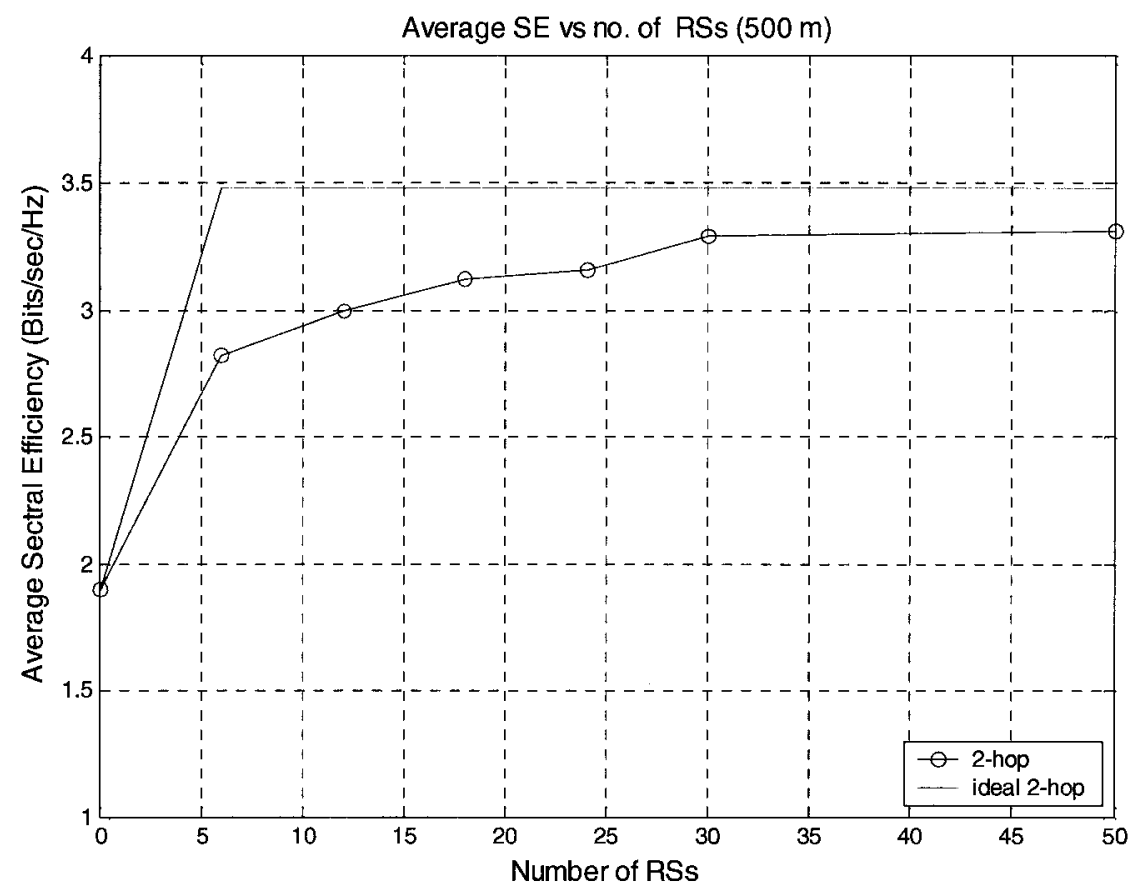

Figure 5.13: Average spectral efficiency for $500 \mathrm{~m}$.

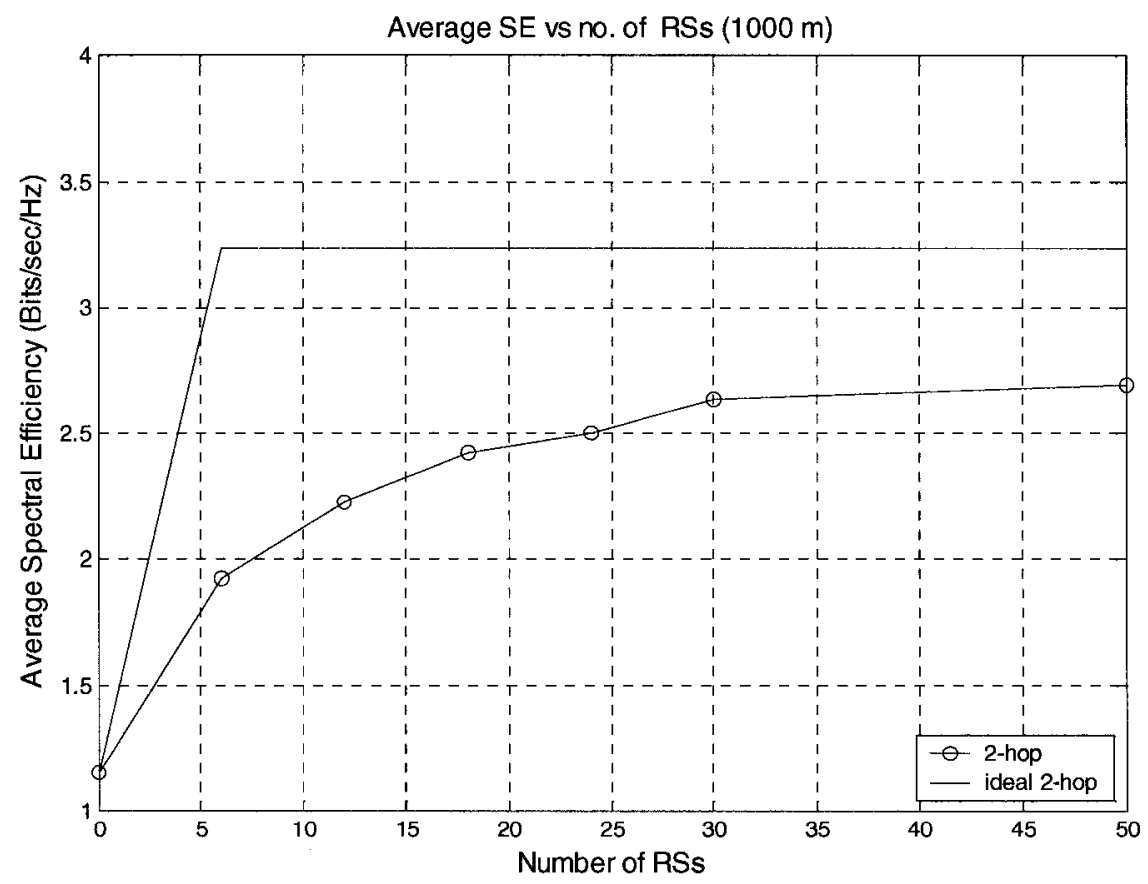

Figure 5.14: Average spectral efficiency for $1000 \mathrm{~m}$. 


\subsection{Outage Comparison for Non-relaying and Relaying Cases}

One of the key benefits of using RSs in the system is to reduce (or if possible to eliminate) the outage in the cell. Figure 5.15 shows the outage benefits of the relaying. For $500 \mathrm{~m}$ cell radius, if relaying is not used, the outage is $39 \%$. However, if $30 \mathrm{RSs}$ are deployed around the $\mathrm{CN}$, the outage is almost eliminated $(0.25 \%)$. For $1000 \mathrm{~m}$ cell radius, if relaying is not used, the outage is $58 \%$. Unlike $500 \mathrm{~m}$ cell radius case, even if 30 RSs are deployed around the $\mathrm{CN}$, the outage cannot be eliminated, but it can be reduced to $4 \%$.

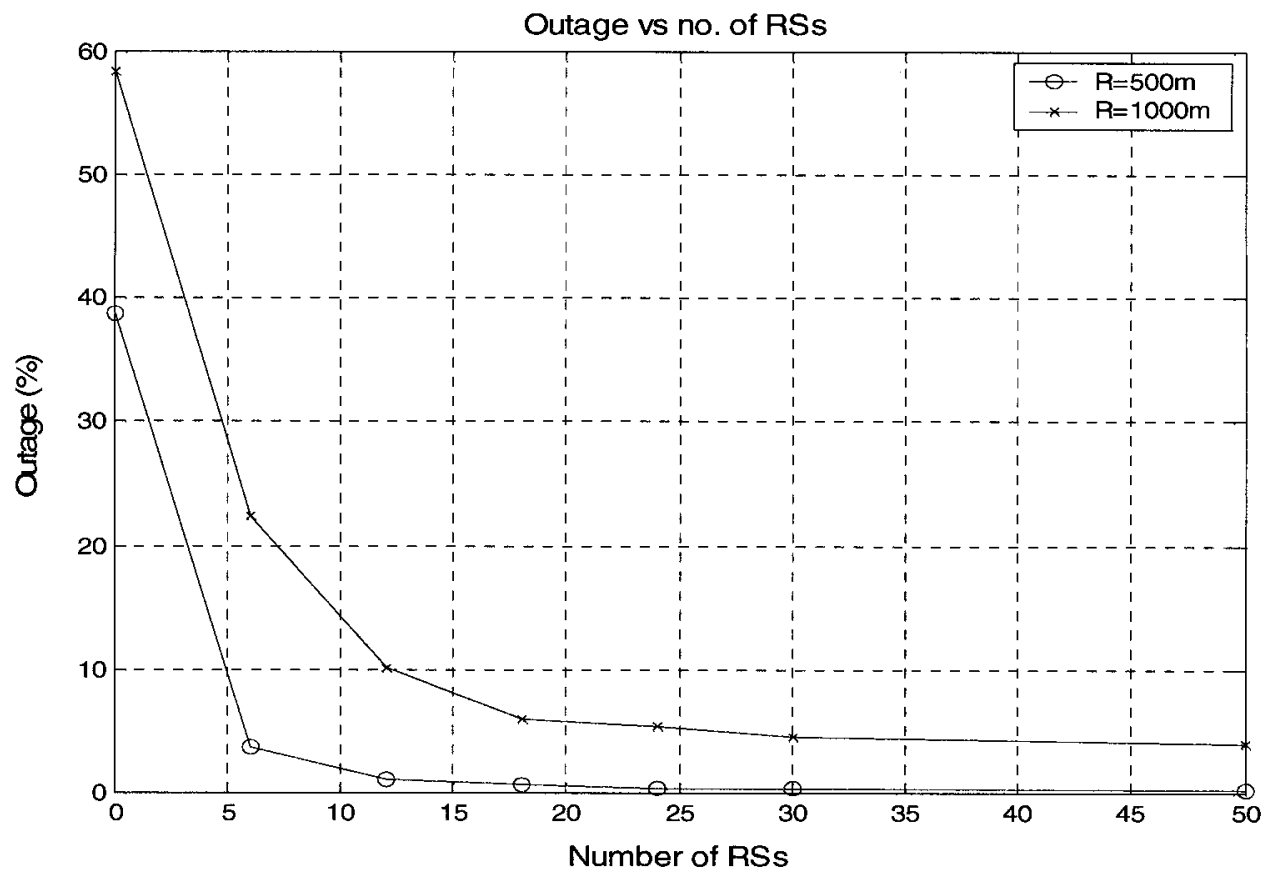

Figure 5.15: Outage for various number of RSs. 


\subsection{Coverage at Various Spectral Efficiency Levels with respect to RS Positions for 30 RSs}

Figures 5.16 and 5.18 illustrate the coverage performance, i.e. the probability of SE being greater than the values given in the horizontal axis, with respect to RS positions for $500 \mathrm{~m}$ and $1000 \mathrm{~m}$ cell sizes, respectively, with $30 \mathrm{RSs}$. The simulation results show that the best distances for RS deployment are $338 \mathrm{~m}$ and $676 \mathrm{~m}$, which are obtained from the analytical derivations in Chapter 2, for $500 \mathrm{~m}$ and $1000 \mathrm{~m}$ cell sizes, respectively. Another interesting observation is that, the performance of (30 RS) $200 \mathrm{~m}$ case in Figure 5.16 is very similar to that of 6 RS (338 m) case in Figure 5.11. In other word, deploying RSs at improper locations gives the same result as using less number of RSs.

Figures 5.17 and 5.19 show the same graphs with a larger scale. For $500 \mathrm{~m}$ case, even though $338 \mathrm{~m}$ is the best distance, the RSs can be deployed at a distance between $320 \mathrm{~m}$ and $380 \mathrm{~m}$ and almost same performance can be obtained. Similarly, for $1000 \mathrm{~m}$ case, the RSs can be deployed at a distance between $650 \mathrm{~m}$ and $720 \mathrm{~m}$. 


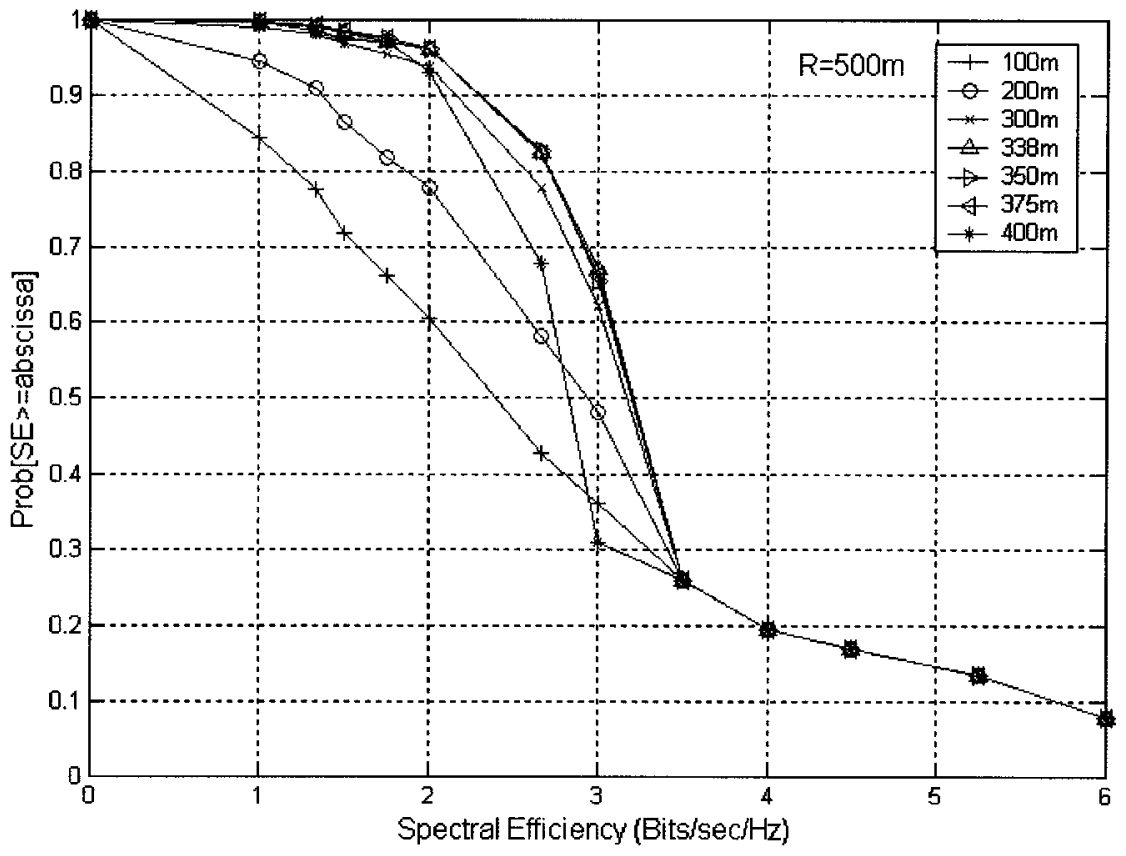

Figure 5.16: Coverage for SE levels wrt RS positions for $500 \mathrm{~m}, 30 \mathrm{RS}$.

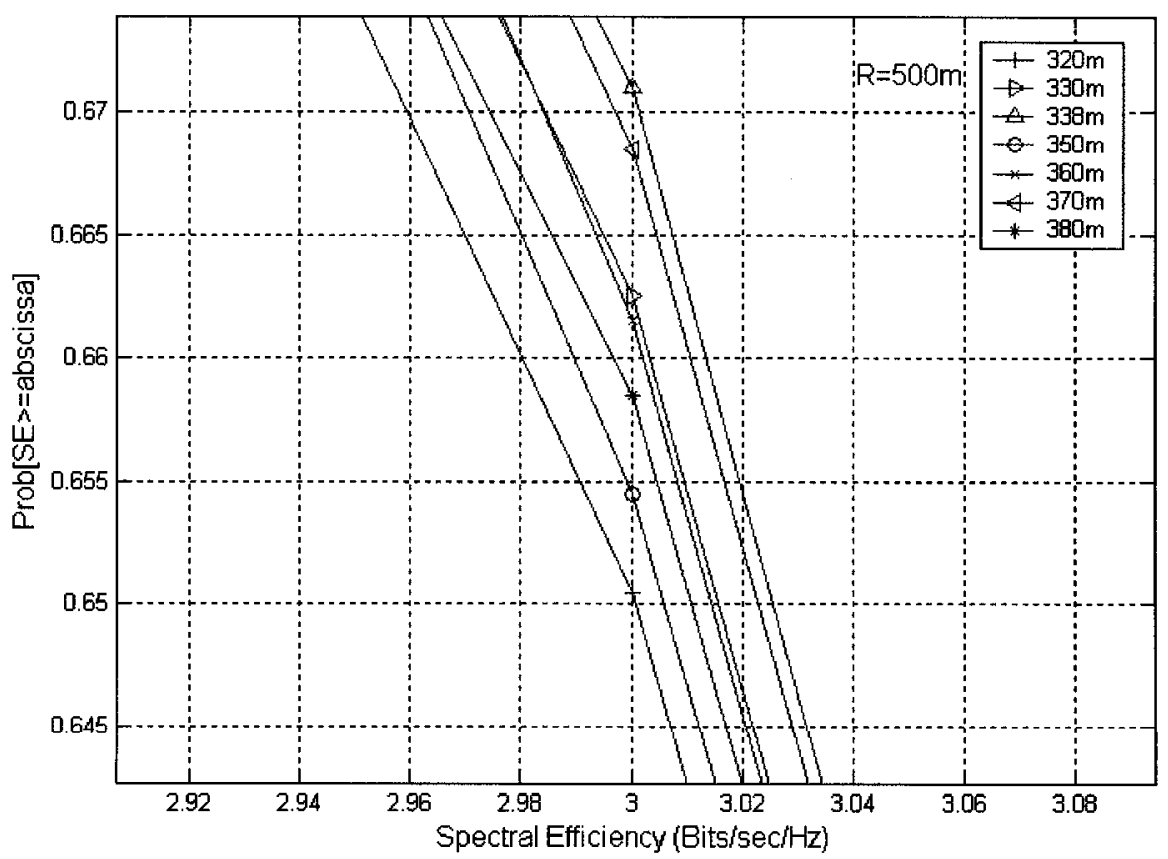

Figure 5.17: (Zoomed) Coverage for SE levels wrt RS positions for $500 \mathrm{~m}, 30 \mathrm{RS}$. 


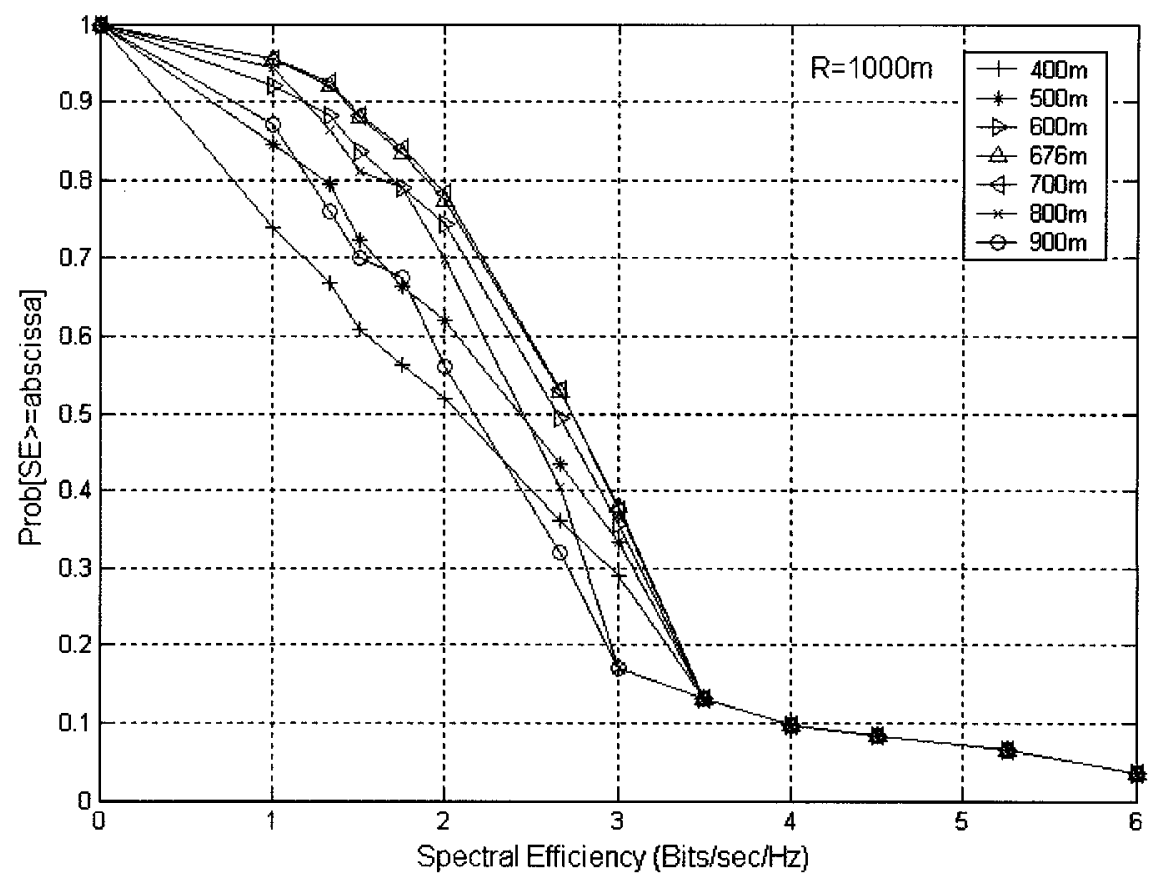

Figure 5.18: Coverage for SE levels wrt RS positions for $1000 \mathrm{~m}, 30 \mathrm{RS}$.

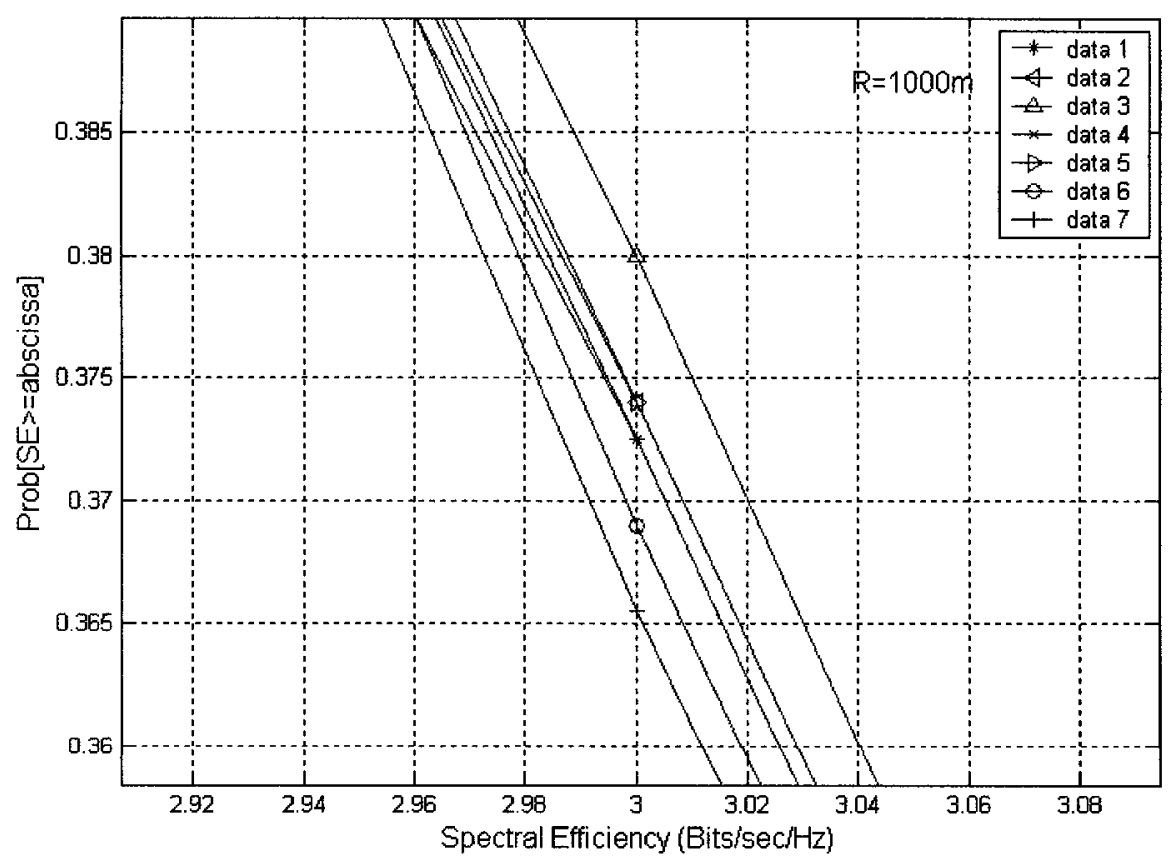

Figure 5.19: (Zoomed) Coverage for SE levels wrt RS positions, 1000 m, 30 RSs. 


\subsection{Coverage at Various Spectral Efficiency Levels with respect to ISF for}

30 RSs

Figures 5.20 and 5.21 depict the coverage performance, i.e. the probability of SE being greater than the values given in the horizontal axis, with respect to interference suppression factor (ISF) for $500 \mathrm{~m}$ and $1000 \mathrm{~m}$ cell sizes, respectively, with 30 RSs. For $500 \mathrm{~m}$ case, if the ISF value is small, e.g. ISF $=0.2$, almost $80 \%$ of the users are provided with spectral efficiency values greater than or equal to $3 \mathrm{bits} / \mathrm{sec} / \mathrm{Hz}$. If the cell size is increased to $1000 \mathrm{~m}$, the effect of ISF decreases due to the fact that larger cells are more noise-limited than the smaller size ones.

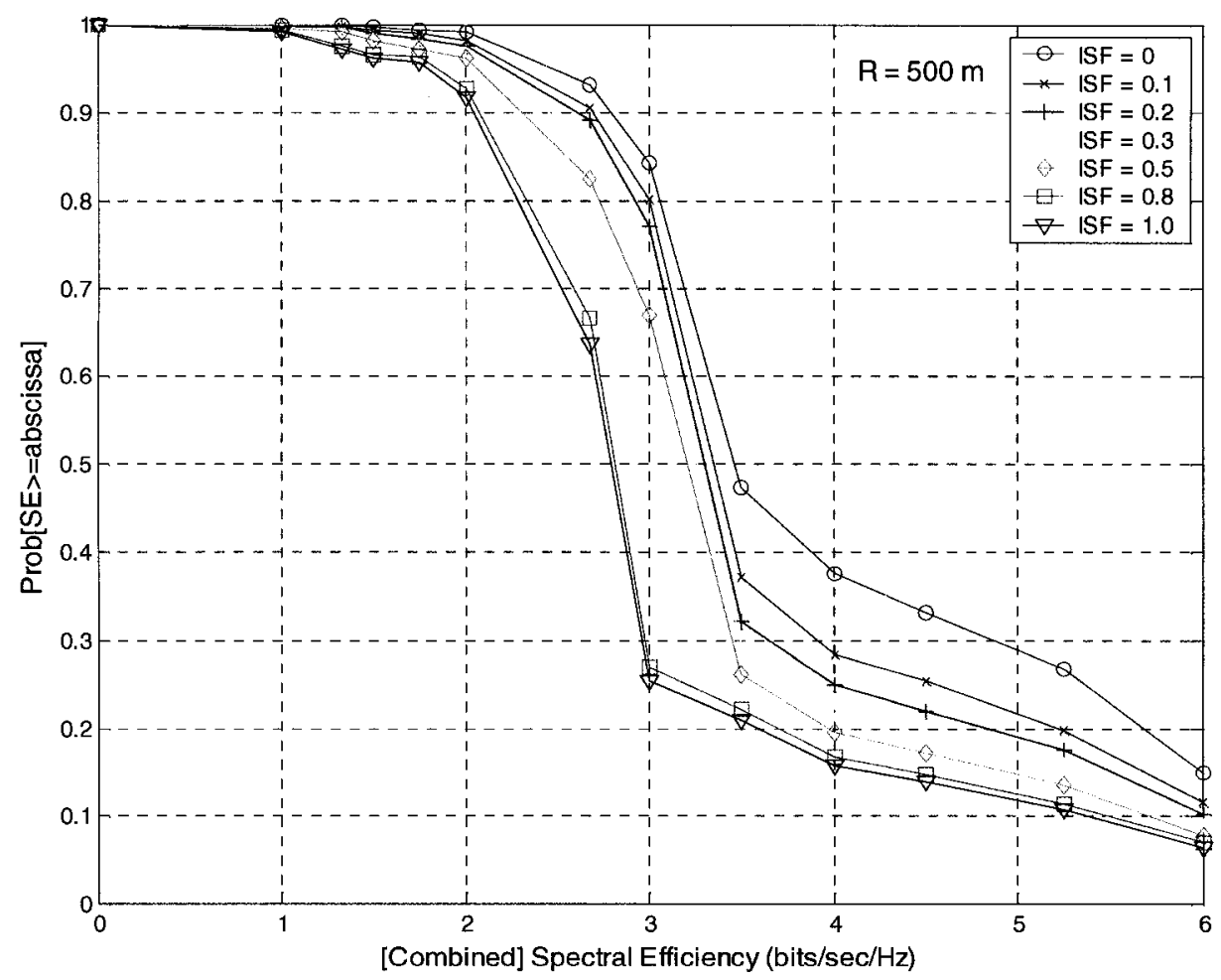

Figure 5.20: Coverage for SE levels wrt ISF, 500 m, 30 RSs. 


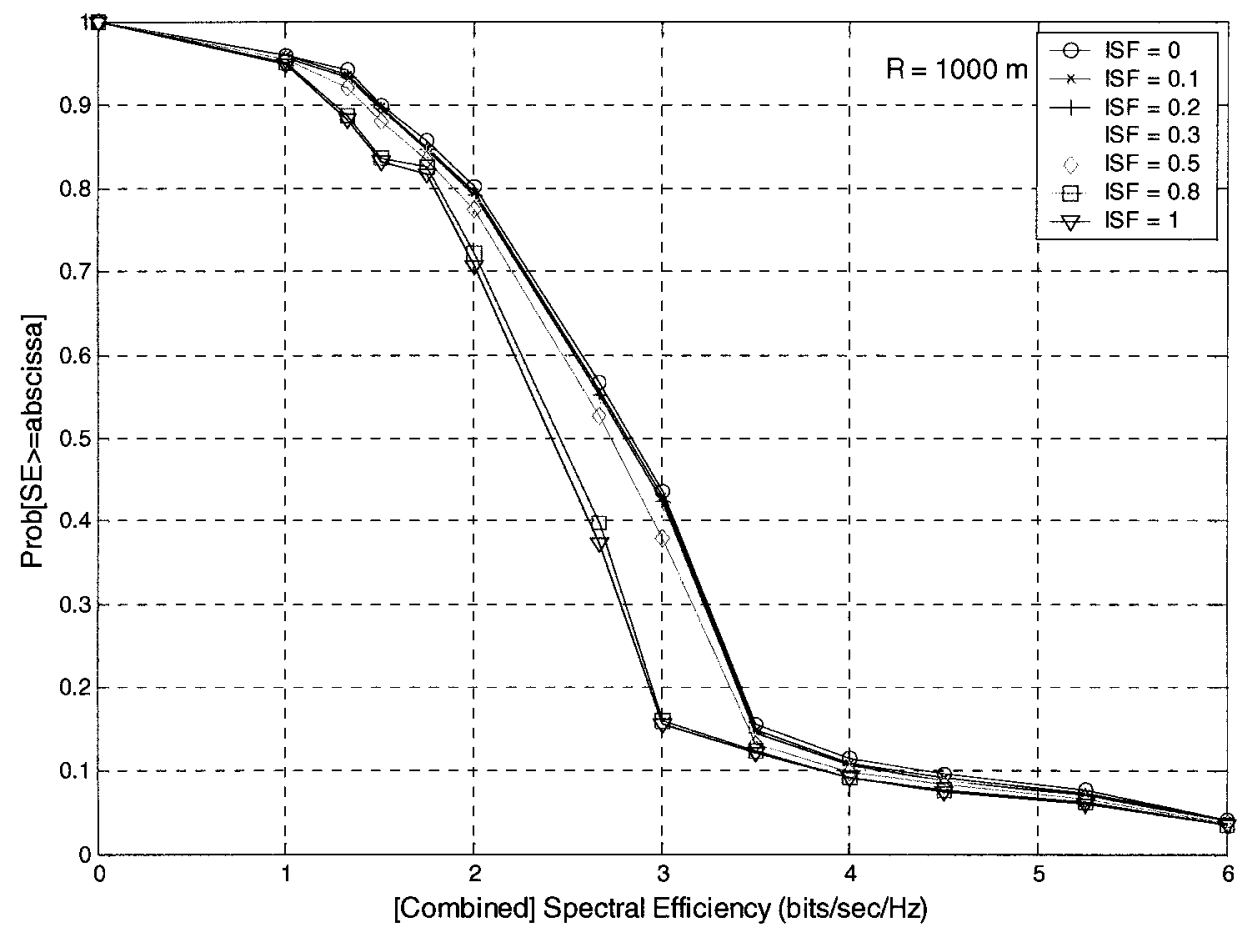

Figure 5.21: Coverage for SE levels wrt ISF, 1000 m, 30 RSs. 


\subsection{Coverage at Various Spectral Efficiency Levels with respect to RS}

\section{Transmit Power}

Figures 5.22 and 5.23 illustrate the coverage performance, i.e. the probability of SE being greater than the values given in the horizontal axis, with respect to the RS transmit power for $500 \mathrm{~m}$ and $1000 \mathrm{~m}$ cell sizes, respectively, with $30 \mathrm{RSs}$. It can be seen that the performances of both cases are improved when the RS transmit power is increased. However, the effect of RS transmit power is more observable for $1000 \mathrm{~m}$ cell size.

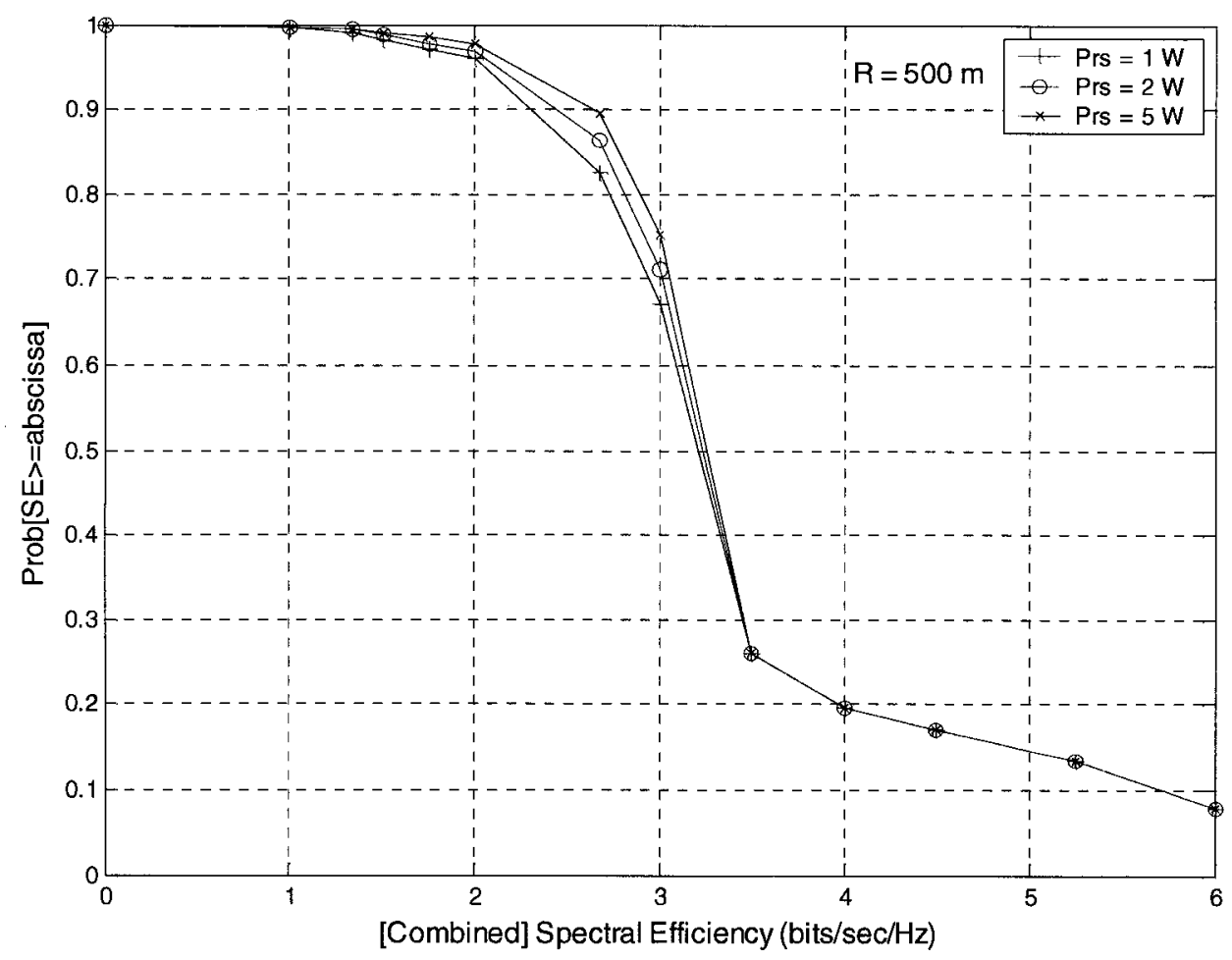

Figure 5.22: Coverage for SE levels wrt RS transmit power, 500 m, 30 RSs. 


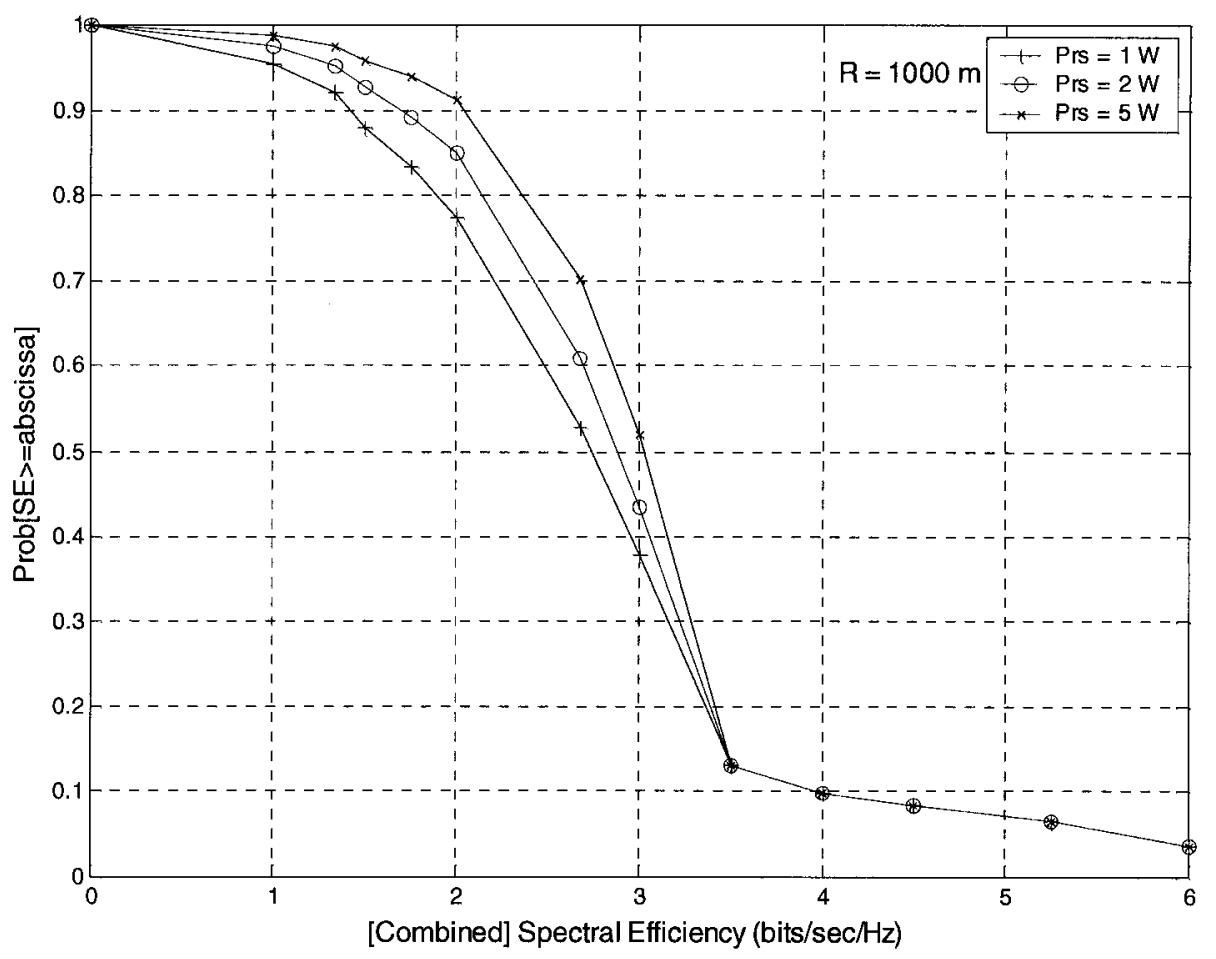

Figure 5.23: Coverage for SE levels wrt RS transmit power, 1000 m, 30 RSs. 


\subsection{Adaptive Modulation and Coding Histogram}

Figures 5.24 and 5.25 show how often spectral efficiency values are used for cell radius of $500 \mathrm{~m}$ and $1000 \mathrm{~m}$, respectively, for non-relaying and $30 \mathrm{RS}$ cases. It is clear that when $30 \mathrm{RSs}$ are used the frequency of the usage of $3 \mathrm{bits} / \mathrm{sec} / \mathrm{Hz}$ (which is the maximum attainable combined spectral efficiency for 2-hop communication) increases. It should also be noticed that, if the quality of the CN-RS links can be improved, the usage of $3 \mathrm{bits} / \mathrm{sec} / \mathrm{Hz}$ increases further.

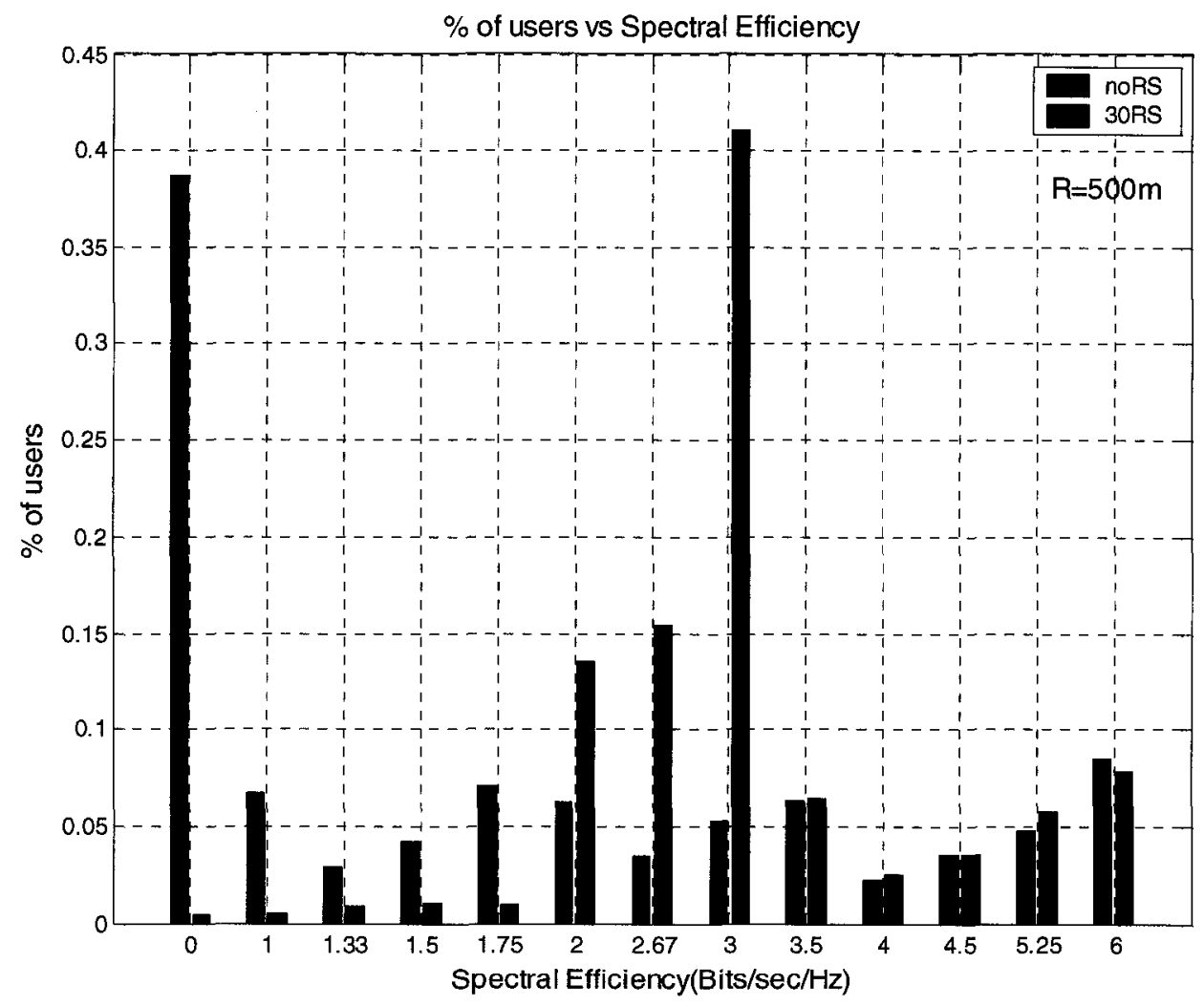

Figure 5.24: Percentage of time using a spectral efficiency, for $500 \mathrm{~m}$. 


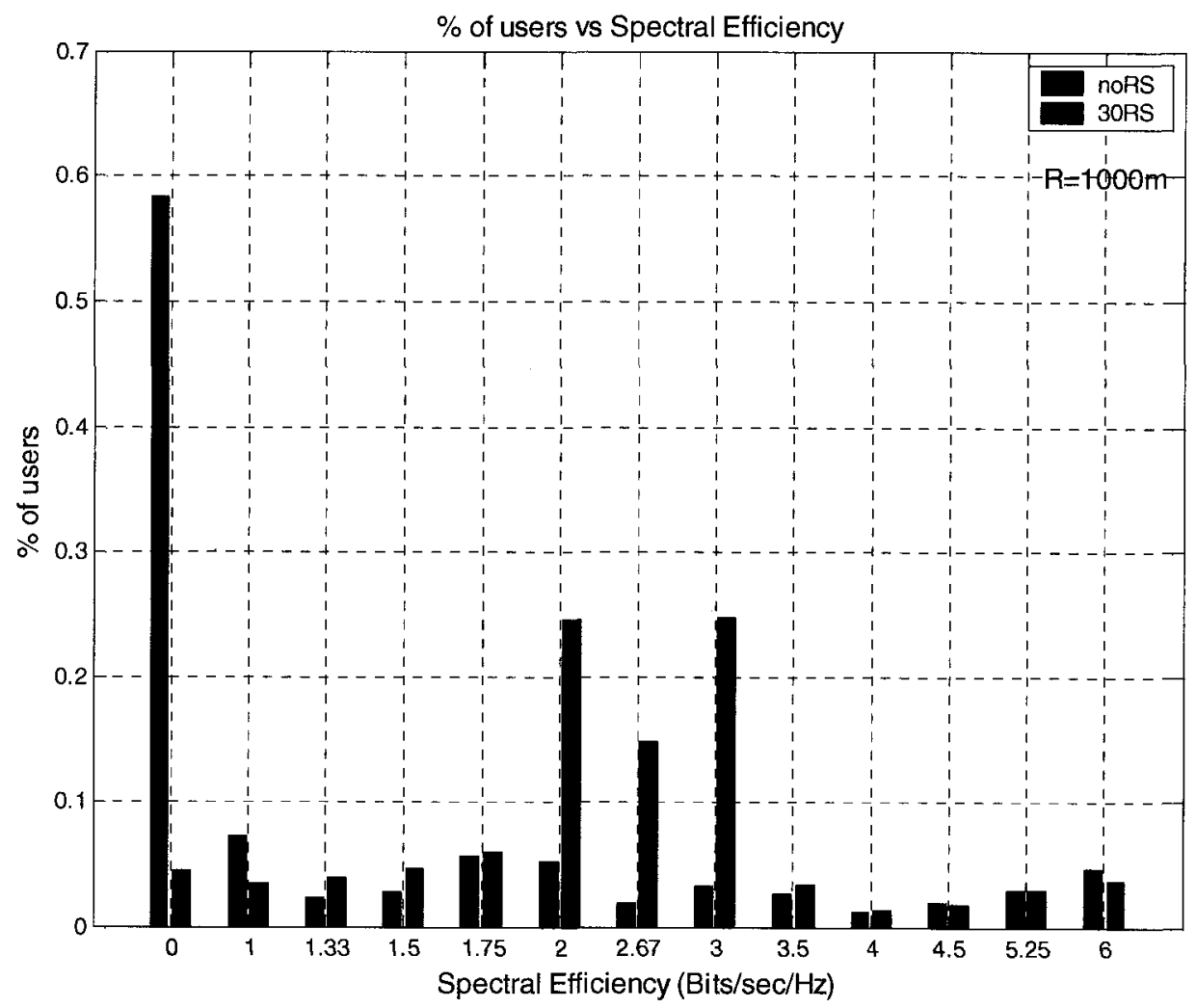

Figure 5.25: Percentage of time using a spectral efficiency, for $1000 \mathrm{~m}$. 


\section{CHAPTER 6: CONCLUSIONS AND DISCUSSIONS}

\subsection{Summary}

This research investigates the potential benefits of employing relays in the cellular networks. In order to support the mathematical analysis, several simulations are done and it is shown that multi-hop relaying concept is a cost-effective solution to the ubiquitous high data rate coverage problem of the future wireless networks.

Firstly, from the analytical results, it is shown that the capacity (or aggregate throughput) of CFRN is directly proportional to the number of root nodes, i.e. first tier relays, deployed around the central node. However, as opposed to the common sense, the capacity of CFRN is independent from the number of hops or relay tiers. Therefore, in order to obtain high capacity from CFRN, the number of root nodes must be increased. The limit for that number depends on the directivity of the beam antenna used for the links between the $\mathrm{CN}$ and the root nodes. It should be noticed that, even though it is possible to obtain higher capacity with CFRN than CCN, if there is a major capacity shortage, it is better to use micro-cellular or pico-cellular networks. After deploying central nodes, RSs should be deployed to eliminate the coverage shortage.

Secondly, for multi-hop relaying, the closest distance where $n$-hop can be utilized is found with analytical derivations. According to those distances and the simple path-loss model used for the calculations, the number and the position of the first tier RSs are 
found in order to provide the maximum attainable spectral efficiency value for the users in the cell for different values of propagation value and relay transmit power. At this point it is observed that the maximum attainable spectral efficiency value for a user varies depending on the number of hops between that user and the $\mathrm{CN}$, i.e. for a user using 2hop communication, the maximum attainable spectral efficiency is half of the maximum available spectral efficiency for the system. For the realistic values of the propagation constant and the relay transmit power, the analytical results, which is summarized in Table 3.4, show that the required number of RSs is around 20. However, for the same conditions, the simulation results show that the required number of RSs for high data rate coverage is around 30 . The difference is due to the effects of lognormal shadowing and adjacent cell interference, which are included in the simulations. From the simulations, it is observed that when the number of RSs is increased from 30 to 50 , the performance gain is negligible and hence for a cost-effective system using 30 RSs is preferable. For 30 RS model, the average spectral efficiency value of $500 \mathrm{~m}$ cell is increased from 1.9 $\mathrm{bits} / \mathrm{sec} / \mathrm{Hz}$ to $3.29 \mathrm{bits} / \mathrm{sec} / \mathrm{Hz}$ and the outage is reduced from $39 \%$ to $0.25 \%$.

Furthermore, as far as the relay position is concerned, the simulations indicate that the optimum distance is the same as the one found from the analytical derivations, i.e. 0.67 times the cell radius, when the transmit power ratio of the $\mathrm{CN}$ and $\mathrm{RS}$ is $10: 1$. Although moving relay position towards or further away from the $\mathrm{CN}$ weakens the system performance, if the relay position stays in the range of 0.64-0.76 times the cell radius, the performance loss is negligible. 


\subsection{Thesis Contributions}

The major contributions of this thesis can be summarized as follows:

- An analytical derivation of the capacity of CFRN in terms of aggregate throughput.

- A comparison of the capacity of CFRN to that of CCN and PCN.

- An analytical derivation of the closest distance from the $\mathrm{CN}$ that n-hop communication can be utilized.

- An analytical derivation (supported by a series of simulations) of the optimum number and distance for first tier RSs in order to obtain ubiquitous high data rate coverage.

- A simulation to show the outage improvement benefit of the multi-hop relaying.

- A simulation to show the average spectral efficiency improvement benefit of the multi-hop relaying.

- A series of simulations that support the idea provided in this thesis that digital fixed relaying technology coupled with the adoption of AMC scheme enhances the throughput and coverage of the cellular networks and realize the ubiquitous high data rate coverage in the entire network. 


\subsection{Future Research}

- Although some of the analytical derivations are done for a general n-hop case, most of the analytical findings and all of the simulation results are for 2-hop case only, which raises a possible future research area where more than 2-hops is studied.

- Load balancing is another interesting point that can be embedded into this research.

- In this research diversity is not used. It is known from [13-16] that diversity can provide a better performance for multi-hop channels, so diversity is another key research area.

- Mobility and hand-off issues can be investigated for future research as well.

- Investigation of realistic traffic model for CFRN may be an interesting research topic. 


\section{References}

[1] W. Mohr, R. Luder, and K. H. Mohrmann, "Data rate estimates, range calculations and spectrum demand for new elements of systems beyond IMT-2000," $5^{\text {th }}$ International Symposium on Wireless Personal Multimedia Communications (WPMC'02), vol. 1, pp. 37-46, 2002.

[2] H. Yanikomeroglu, "Fixed and mobile relaying technologies for cellular networks," 2nd Workshop on Applications and Services in Wireless Networks (ASWN'02), pp. 75-81, 2002.

[3] H. Hu, H. Yanikomeroglu, D. D. Falconer, and Shalini Periyalwar, "Range extension in cellular networks with digital fixed relays," IEEE Globecom'04, December 2004.

[4] R. Pabst, B. H. Walke, D. C. Schultz, P. Herhold, H. Yanikomeroglu, S. Mukherjee, H. Viswanathan, M. Lott, W. Zirwas, M. Dohler, H. Aghvami, D. D. Falconer, and G. P. Fettweis, "Relay-based deployment concepts for wireless and mobile broadband cellular radio", IEEE Communications Magazine, vol. 42, no. 9, pp. 80-89, September 2004.

[5] E. W. Quinn, "The cell enhancer," 36th IEEE Vehicular Technology Conference (VTC'86), pp. 77-83, May 1986.

[6] E. H. Drucker, "Development and application of a cellular repeater," IEEE Vehicular Technology Conference (VTC'88), pp. 321-325, June 1988.

[7] W. T. Slingsby and J. P. McGeehan, "A high-gain cell enhancer," IEEE Vehicular Technology Conference (VTC'92), pp. 756-758, May 1992.

[8] A. A. Baghai and J. Divall, "Cost effective solutions for coverage enhancement," Seventh IEE European Conference on Mobile and Personal Communications, pp. 5156, December 1993.

[9] T. J. Harrold and A. R. Nix, "Intelligent relaying for future personal communications systems," IEE Colloquium on Capacity and Range Enhancement Techniques for the Third Generation Mobile Communications and Beyond (Ref. No. 2000/003), pp. 9/1$9 / 5$, February 2000

[10] G. N. Aggelou and R. Tafazolli, "On the relaying capability of next generation GSM cellular networks," IEEE Personal Communications, pp. 40-47, February 2001. 
[11] H. Wu, C. Qiao, and O. Tonguz, "Performance analysis of iCAR (Integrated cellular and ad-hoc relay system)," IEEE International Conference on Communications (ICC'01), vol. 2, pp. 450-455, 2001.

[12] Y. D. Lin and Y. C. Hsu, "Multihop cellular: a new architecture for wireless communications," IEEE INFOCOM'00, pp. 1273-1282, 2000.

[13] V. Sreng, H. Yanikomeroglu, and D. D. Falconer, "Relayer selection strategies in cellular networks with peer-to-peer relaying," IEEE Vehicular Technology Conference Fall 2003 (VTC'F03), October 2003.

[14] V. Sreng, H. Yanikomeroglu, and D. D. Falconer, "Capacity enhancement through two-hop relaying in cellular radio systems," IEEE Wireless Communications and Networking Conference (WCNC'02), March 2002.

[15] J. Boyer, D. D. Falconer, and H. Yanikomeroglu, "Multihop diversity in wireless relaying channels," IEEE Transactions on Communications, vol. 52, no. 10, pp. 18201830, October 2004.

[16] N. Esseling, "Extending the Range of HiperLAN/2 Cells in Infrastructure Mode using Forward Mobile Terminals," European Personal Mobile Communication Conference (EPMCC'01), p. Session 23, February 2001.

[17] N. Esseling, B. Walke, and R. Pabst, "Performance Evaluation of a Fixed Relay Concept for Next Generation Wireless Systems," Personal Indoor and Mobile Radio Communications (PIMRC'04), pp. 9, September 2004.

[18] D. C. Schultz, R. Pabst, and T. Irnich, "Multi-hop based Radio Network Deployment for efficient Broadband Radio Coverage," Wireless Personel Multimadia Communications (WPMC'04), vol. 2, pp. 377-381, October 2003.

[19] C. Qiao, H. Wu, and O. Tonguz, "Load balancing via relay in next generation wireless systems," Mobile and Ad Hoc Networking and Computing Conference (MobiHOC'00), 2000.

[20] E. Armanious, D. D. Falconer, and H. Yanikomeroglu, "Adaptive modulation, adaptive coding, and power control for fixed cellular broadband wireless systems," IEEE Wireless Communications and Networking Conference (WCNC'03), March 2003. 


\section{Appendix A - Definitions of Some Terms in Tree Structure}

Figure A.1 shows the central node and nodes which belong to a binary tree. In the figure

- node ' 1 ' represents the root node of the tree,

- node ' 2 ' is the parent node of the nodes ' 3 ' and ' 4 ',

- nodes ' 3 ' and '4' are the child nodes of node ' 2 '.

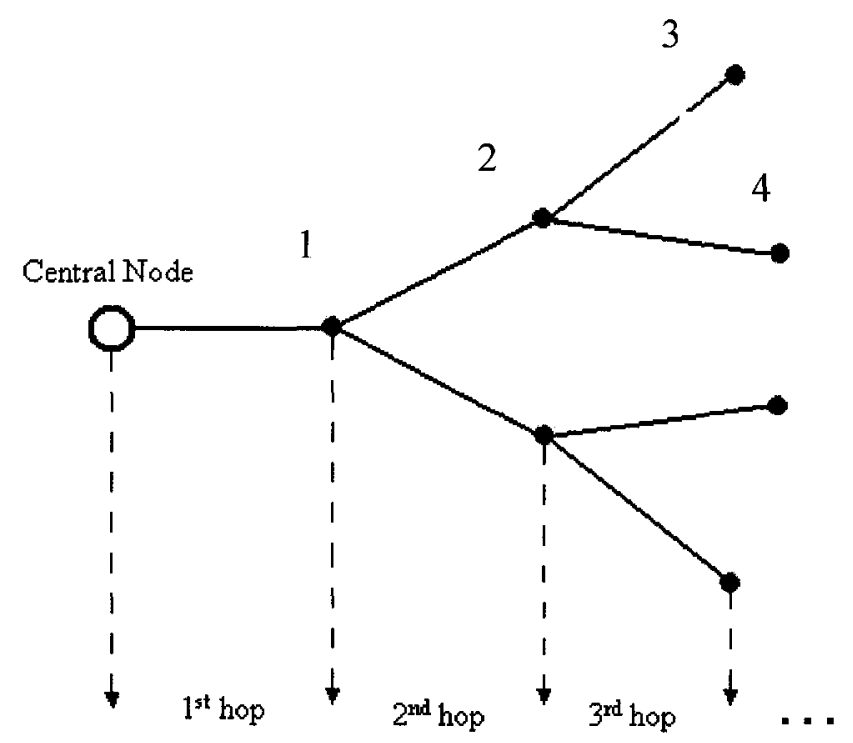

Figure A.1: Central node and nodes belong to a binary tree. 


\section{Appendix B - Proof of (2.17)}

Equation (2.16) is derived according to the tree structure in Figure 2.2, a binary tree. Equation (2.15) can be rewritten as follows:

$$
R_{C R N} \approx\left(\frac{\text { No. of nodes in the tree }}{\left(\text { No. of channel groups for } 1^{\text {st }} \text { hop }\right)+\left(\text { No. of channel groups for } 2^{\text {nd }} \text { hop }\right)+1}\right)\left(M R_{W}\right)
$$

The number of nodes in the tree is independent of the structure of the tree and assumed to be equal to $N$. The number of channel groups needed for the first hop link is equal to the number of nodes in the tree since this link carries the traffic of all the nodes in the tree; therefore it is equal to $N$. It is assumed that each node has separate directional antennas for each node-to-node links, enabling the reuse of the same channel groups. In Figure 2.2, there are two second hop links, each carrying the traffic of $(N-1) / 2$ nodes and they both use the same channel groups, so total number of channel groups for the second hop links is equal to $(N-1) / 2$. In the general case, if each root node has $p$ child nodes, then there are $p$ second hop links, each carrying the traffic of $(N-1) / p$ nodes. Since each second hop link uses separate directional antennas, total number of channel groups needed for the second hop links is equal to $(N-1) / p$. Inserting these values to the above equation, for the limit case,

$$
R_{C R N} \approx\left(\lim _{N \rightarrow \infty} \frac{N}{N+\left\lceil\frac{N-1}{p}\right\rceil+1}\right)\left(M R_{W}\right) \Rightarrow R_{C R N} \approx \frac{p}{p+1}\left(M R_{W}\right) .
$$




\section{Appendix C - All Orthogonal Channel Groups for All the Links in a}

\section{Tree}

In the analytical process of (2.29), it is still assumed that two child nodes can use the same frequency band since their parent node has two separate narrow beam antenna. If this assumption is removed, then the total number of channel groups can be calculated as

$$
\begin{aligned}
N_{T} & =N+\sum_{k=2}^{q}\left(N-\left(\frac{p^{k-1}-1}{p-1}\right)\right)+N=N+N \sum_{k=2}^{q} 1-\sum_{k=2}^{q} \frac{p^{k-1}-1}{(p-1)}+N \\
& =N+N(q-1)-\frac{1}{p-1} \sum_{k=2}^{q} p^{k-1}+\frac{q-1}{p-1}+N .
\end{aligned}
$$

In the above, we assumed that all the nodes use orthogonal channel groups in their coverage region as well. Then, $R_{C F R N}$ can be obtained as

$$
\begin{aligned}
R_{C R N} & \approx\left[\lim _{N \rightarrow \infty}\left(\frac{N}{N+N(q-1)-\frac{1}{p-1} \sum_{k=2}^{q} p^{k-1}+\frac{q-1}{p-1}+N}\right)\right]\left(M R_{W}\right)=\frac{1}{q} M R_{W} \\
& =\frac{1}{\log _{p}(N+1)+1} M R_{W} .
\end{aligned}
$$




\section{Appendix D - Details of the Ideal 2-hop Case}

For a 2-hop communication system, the users having spectral efficiencies greater than or equal to the half of the maximum available spectral efficiency level (in our case 3 bits $/ \mathrm{sec} / \mathrm{Hz}$ ) do not need relay assistance and hence deploying more and more RSs does not improve their performance. On the other hand, the performance of the users having spectral efficiencies less than $3 \mathrm{bits} / \mathrm{sec} / \mathrm{Hz}$ can be improved with relay assistance. When 2-hop relay assistance is used for a user, the maximum attainable spectral efficiency level is $3 \mathrm{bits} / \mathrm{sec} / \mathrm{Hz}$. The maximum performance of 2-hop is obtained if the users having spectral efficiencies less than $3 \mathrm{bits} / \mathrm{sec} / \mathrm{Hz}$ are provided with relay assistance and attain a spectral efficiency level of $3 \mathrm{bits} / \mathrm{sec} / \mathrm{Hz}$. Therefore, for the ideal 2-hop case, the lowest spectral efficiency level is $3 \mathrm{bits} / \mathrm{sec} / \mathrm{Hz}$.

Figures 5.11 and 5.12 can be used to calculate the average spectral efficiency level of 2-hop communication for $500 \mathrm{~m}$ and $1000 \mathrm{~m}$ cell sizes, respectively. Table A.1 shows the usage of the spectral efficiency levels for $500 \mathrm{~m}$ cell size and non-relaying case.

Table A.1: The percentage of the usage of the Spectral Efficiency levels.

\begin{tabular}{|c|c|c|c|c|c|c|c|c|c|c|c|c|c|}
\hline SE & 6 & 5.25 & 4.5 & 4 & 3.5 & 3 & 2.67 & 2 & 1.75 & 1.5 & 1.33 & 1 & $<1$ \\
\hline \% usage & 8.5 & 4.8 & 3.6 & 2.25 & 6.35 & 5.3 & 3.45 & 6.25 & 7.05 & 4.2 & 2.85 & 6.7 & 38.7 \\
\hline
\end{tabular}

Then, the average spectral efficiency level for this case can be calculated as 


$$
S E_{a v_{-} n o R S}=\left(\begin{array}{l}
6 \times 8.5+5.25 \times 4.8+4.5 \times 3.6+4 \times 2.25+ \\
3.5 \times 6.35+3 \times 5.3+2.67 \times 3.45+ \\
2 \times 6.25+1.75 \times 7.05+1.5 \times 4.2+ \\
1.33 \times 2.85+1 \times 6.7+0 \times 38.7
\end{array}\right) / 100=1.9 \mathrm{bits} / \mathrm{sec} / \mathrm{Hz}
$$

For the ideal 2-hop case, the usage of the spectral efficiency values higher than or equal to $3.5 \mathrm{bits} / \mathrm{sec} / \mathrm{Hz}$ is same as the non-relaying case. However, users having spectral efficiency values less than $3 \mathrm{bits} / \mathrm{sec} / \mathrm{Hz}$ are assumed to be provided with $3 \mathrm{bits} / \mathrm{sec} / \mathrm{Hz}$ via relaying. Table A.2 shows the usage of the spectral efficiency levels for $500 \mathrm{~m}$ cell size and ideal 2-hop case.

Table A.2: The percentage of the usage of the spectral efficiency levels for ideal 2-hop case.

\begin{tabular}{|c|c|c|c|c|c|c|}
\hline SE & 6 & 5.25 & 4.5 & 4 & 3.5 & 3 \\
\hline$\%$ usage & 8.5 & 4.8 & 3.6 & 2.25 & 6.35 & 74.5 \\
\hline
\end{tabular}

$$
S E_{a v_{-} 2-h o p}=\left(\begin{array}{l}
6 \times 8.5+5.25 \times 4.8+4.5 \times 3.6+ \\
4 \times 2.25+3.5 \times 6.35+3 \times 74.5
\end{array}\right) / 100=3.47 \mathrm{bits} / \mathrm{sec} / \mathrm{Hz} .
$$

These two spectral efficiency values are used to obtain the ideal 2-hop curve in Figure 5.13. 